\title{
Iterative Co-channel Interference Suppression
}

\author{
Chaowen $\mathrm{Gu}$
}

\author{
A thesis submitted to \\ the Faculty of Graduate Studies and Research \\ in partial fulfillment of the requirements for the degree of \\ Master of Applied Science
}

Ottawa-Carleton Institute for Electrical and Computer Engineering Department of Systems and Computer Engineering

Carleton University

Ottawa, Ontario, Canada

July 25,2007 


$\begin{array}{ll}\begin{array}{l}\text { Library and } \\ \text { Archives Canada }\end{array} & \begin{array}{l}\text { Bibliothèque et } \\ \text { Archives Canada }\end{array} \\ \begin{array}{l}\text { Published Heritage } \\ \text { Branch }\end{array} & \begin{array}{l}\text { Direction du } \\ \text { Patrimoine de l'édition }\end{array} \\ \begin{array}{l}\text { 395 Wellington Street } \\ \text { Ottawa ON K1A ON4 }\end{array} & \begin{array}{l}\text { 395, rue Wellington } \\ \text { Ottawa ON K1A ON4 } \\ \text { Canada }\end{array}\end{array}$

Your file Votre référence ISBN: 978-0-494-33650-2 Our file Notre référence ISBN: 978-0-494-33650-2

NOTICE:

The author has granted a nonexclusive license allowing Library and Archives Canada to reproduce, publish, archive, preserve, conserve, communicate to the public by telecommunication or on the Internet, loan, distribute and sell theses worldwide, for commercial or noncommercial purposes, in microform, paper, electronic and/or any other formats.

The author retains copyright ownership and moral rights in this thesis. Neither the thesis nor substantial extracts from it may be printed or otherwise reproduced without the author's permission.
AVIS:

L'auteur a accordé une licence non exclusive permettant à la Bibliothèque et Archives Canada de reproduire, publier, archiver, sauvegarder, conserver, transmettre au public par télécommunication ou par l'Internet, prêter, distribuer et vendre des thèses partout dans le monde, à des fins commerciales ou autres, sur support microforme, papier, électronique et/ou autres formats.

L'auteur conserve la propriété du droit d'auteur et des droits moraux qui protège cette thèse. $\mathrm{Ni}$ la thèse ni des extraits substantiels de celle-ci ne doivent être imprimés ou autrement reproduits sans son autorisation.
In compliance with the Canadian

Privacy Act some supporting forms may have been removed from this thesis.

While these forms may be included in the document page count, their removal does not represent any loss of content from the thesis.
Conformément à la loi canadienne sur la protection de la vie privée, quelques formulaires secondaires ont été enlevés de cette thèse.

Bien que ces formulaires aient inclus dans la pagination, il n'y aura aucun contenu manquant.

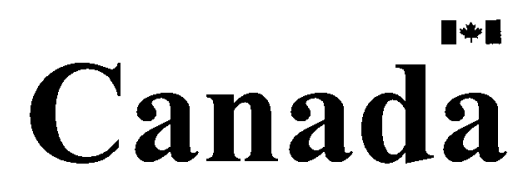




\begin{abstract}
In this thesis, we propose a fully asynchronous iterative receiver for co-channel interference (CCI) suppression. We consider the downlink of a cellular system as our model, where signals from neighboring base stations cause the interference. To handle asynchronously received code words, our receiver suppresses the CCI over frames spanning several code words. The BER performance results show that the asynchronous system suffers only a minor penalty compared to its fully synchronous counterpart. Cellular system simulation results show that our receiver can effectively reduce the co-channel interference $(\mathrm{CCI})$, thereby improving the system performance in terms of throughput and outage probability.
\end{abstract}




\section{Contents}

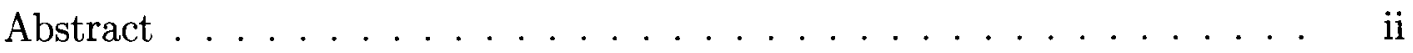

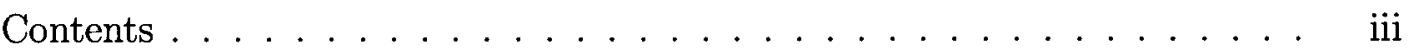

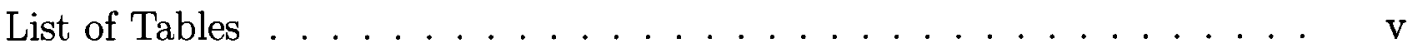

List of Figures . . . . . . . . . . . . . . . . . . . vii

List of Symbols . . . . . . . . . . . . . . . . . . viii

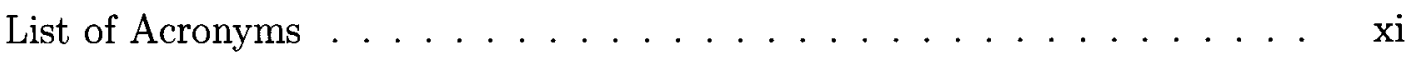

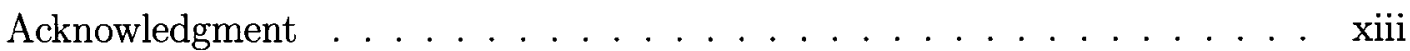

1 Introduction 1

1.1 Review of CCI Mitigation . . . . . . . . . . . . . . 2

1.2 Review of ISI Elimination $\ldots \ldots \ldots \ldots$

1.3 Multi-user Detection for ISI Channels . . . . . . . . . . . . . 7

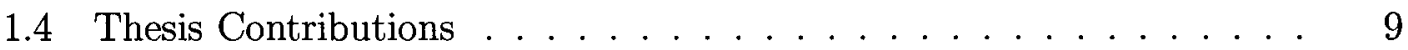

1.5 Thesis Organization . . . . . . . . . . . . . . . . . . 11

2 System Model Description $\quad 12$

2.1 Transmitter . . . . . . . . . . . . . . . . . . . . . 13

2.2 Channel Model . . . . . . . . . . . . . . . . . . . 15

2.3 Receiver . . . . . . . . . . . . . . . . . . . . . . 19

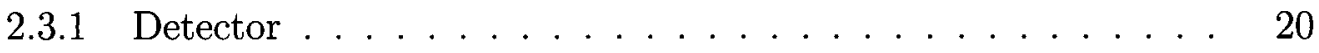

2.3 .2 Decoder . . . . . . . . . . . . . . . . . 23

2.3.3 Feedback Subsystem . . . . . . . . . . . . . . . . 25

2.4 Frame Processing . . . . . . . . . . . . . . . . . . . 28

2.5 Novel Contributions of the System Model . . . . . . . . . . . . . 29

3 System Performance 31

3.1 System Performance for SUI-5 Channel . . . . . . . . . . . . . . 32

3.1 .1 Performance for $N_{S}=2, N_{A}=1 \ldots \ldots \ldots \ldots \ldots \ldots$

3.1.2 Performance for $N_{S}=2, N_{A}=2 \ldots \ldots \ldots \ldots \ldots$. . . 40

3.1.3 Performance for $N_{S}=3, N_{A}=2 \ldots \ldots \ldots \ldots \ldots \ldots$ 
3.2 System Performance for 5 -tap Channel . . . . . . . . . . . 48

3.2.1 Performance for $N_{S}=2, N_{A}=1 \ldots \ldots \ldots \ldots \ldots$

3.2.2 Performance for $N_{S}=2, N_{A}=2$ and $N_{S}=3, N_{A}=2 \ldots \ldots$

3.3 System Performance for Rich Scattering Channel . . . . . . . . 54

3.3.1 Performance for $N_{S}=2, N_{A}=1 \ldots \ldots \ldots \ldots \ldots$

3.3.2 Performance for $N_{S}=2, N_{A}=2$ and $N_{S}=3, N_{A}=2 \ldots \ldots$

4 Cellular Simulation Results $\quad 62$

4.1 Cellular Model Description . . . . . . . . . . . . . . . 63

4.2 Simulation Results of Connection Rates . . . . . . . . . . . . 68

4.3 Simulation Results of Throughput . . . . . . . . . . . . 88

5 Conclusions and Future Work $\quad 93$

5.1 Conclusions . . . . . . . . . . . . . . . . . . . . 93

5.2 Future Work . . . . . . . . . . . . . . . . . . . . . 94

A Detector Parameter Calculation $\quad 95$

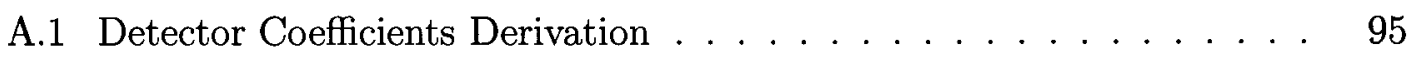

A.2 Mean and Variance of $z_{n}^{(s)} \ldots \ldots \ldots \ldots \ldots \ldots$

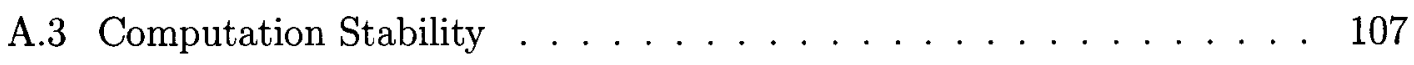

$\begin{array}{ll}\text { References } & 109\end{array}$ 


\section{List of Tables}

2.1 Power delay profile of SUI-5 channel. . . . . . . . . . . . . . 16

3.1 Shared simulation parameters. . . . . . . . . . . . . . 32

4.1 Cellular system parameters. . . . . . . . . . . . . . . 64

4.2 Connection rate on SUI-5 channel with $N_{S}=2, N_{A}=1 . \quad \ldots . . .69$

4.3 Average connection rate and average throughput, IC case. . . . . . . 90 


\section{List of Figures}

$1.1 \quad$ CCI examples. . . . . . . . . . . . . . . . . 3

1.2 Block diagram of a turbo equalizer $\ldots \ldots \ldots \ldots \ldots$

1.3 Uplink of a SDMA system. . . . . . . . . . . . . . . . 8

1.4 Block diagram of an iterative multi-user detector. . . . . . . . 8

2.1 Block diagram of the MIMO system model. . . . . . . . . . 12

2.2 Block diagram of a transmitter. . . . . . . . . . . . . . . 13

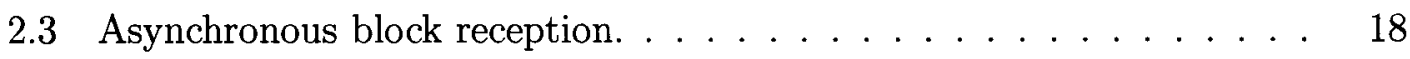

2.4 Block diagram of a MIMO detector. . . . . . . . . . . . . 20

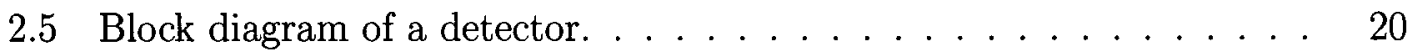

2.6 Block diagram of a decoder. . . . . . . . . . . . . . . 23

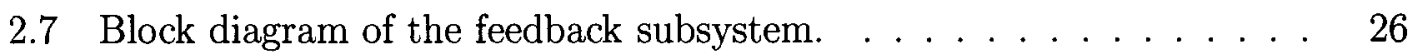

3.1 SUI-5 power delay profile. . . . . . . . . . . . . . . . 32

3.2 Error performance for different modes of synchronization on the SUI- 5 channel with $N_{S}=2, N_{A}=1, \Delta=0 \mathrm{~dB} \ldots \ldots \ldots \ldots \ldots$

3.3 Block 1 BER performance of different frame structures on SUI-5 channel with $N_{S}=2, N_{A}=1, \Delta=0 \mathrm{~dB} \ldots \ldots \ldots \ldots \ldots \ldots \ldots$

3.4 Performance on SUI-5 channel with $N_{S}=2, N_{A}=1, \Delta=0 \mathrm{~dB}$. . . $\quad 36$

3.5 Performance on SUI-5 channel with $N_{S}=2, N_{A}=1, \Delta=9 \mathrm{~dB}$. . . 38

3.6 BER performance on SUI-5 channel with $N_{S}=2, N_{A}=1 \ldots \ldots 41$

3.7 BER performance on SUI-5 channel with $N_{S}=2, N_{A}=1 \ldots \ldots .41$

3.8 BER performance on SUI-5 channel with $N_{S}=2, N_{A}=1 \ldots \ldots 42$

3.9 BER performance on SUI-5 channel with $N_{S}=2, N_{A}=1 \ldots \ldots .42$

3.10 Performance on SUI-5 channel with $N_{S}=2, N_{A}=2, \Delta=0 \mathrm{~dB} . \quad \ldots \quad 43$

3.11 Performance on SUI-5 channel with $N_{S}=2, N_{A}=2, \Delta=3 \mathrm{~dB}$. . . $\quad 44$

3.12 Performance on SUI-5 channel with $N_{S}=3, N_{A}=2, \Delta=0 \mathrm{~dB}$. . . 46

3.13 Performance on SUI- 5 channel with $N_{S}=3, N_{A}=2, \Delta=3 \mathrm{~dB} . \quad \ldots \quad 47$

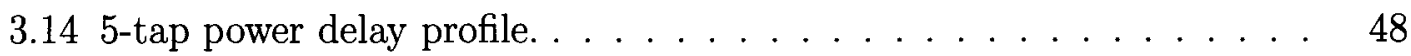

3.15 Performance on 5-tap channel with $N_{S}=2, N_{A}=1, \Delta=0 \mathrm{~dB} . \ldots \quad 49$ 
3.16 Performance on 5-tap channel with $N_{S}=2, N_{A}=1, \Delta=6 \mathrm{~dB} \ldots \ldots \quad 51$

3.17 Performance on 5-tap channel with $N_{S}=2, N_{A}=2, \Delta=0 \mathrm{~dB}$. . . 52

3.18 Performance on 5-tap channel with $N_{S}=3, N_{A}=2, \Delta=0 \mathrm{~dB}$. . . 53

3.19 Rich Scattering channel power delay profile. . . . . . . . . . . 54

3.20 Performance on Rich Scattering channel with $N_{S}=2, N_{A}=1, \Delta=0$ dB. . . . . . . . . . . . . . . . . . 56

3.21 Performance on Rich Scattering channel with $N_{S}=2, N_{A}=1, \Delta=12$ dB . . . . . . . . . . . . . . . . . 57

3.22 BER performance of "no IC" schemes on Rich Scattering channel with $N_{S}=2, N_{A}=1, \Delta=12 \mathrm{~dB} \ldots \ldots \ldots \ldots$

3.23 Performance on Rich Scattering channel with $N_{S}=2, N_{A}=2, \Delta=0$

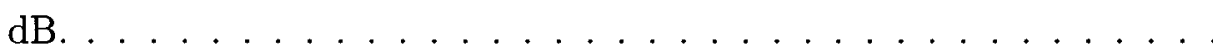

3.24 Performance on Rich Scattering channel with $N_{S}=3, N_{A}=2, \Delta=0$ $\mathrm{dB} \ldots \ldots \ldots \ldots \ldots$. . . . . . . . . . . . . . . . . .

4.1 Transmit antenna gain for sectoring. . . . . . . . . . . . . . 65

4.2 Cellular layout. . . . . . . . . . . . . . . . 67

4.33 -D connection rate graph on SUI-5 channel with $N_{S}=2, N_{A}=1$. . 70

4.4 Connection rate on SUI-5 channel with $N_{S}=2, N_{A}=1 . \ldots 70$

4.5 Scheme 111 (cell reuse factor $=1$, sectors $/$ cell $=1$, sector reuse factor $=1$ ). 73

4.6 Scheme 131 (cell reuse factor $=1$, sectors $/$ cell $=3$, sector reuse factor $=1$ ). 74

4.7 Scheme 161 (cell reuse factor $=1$, sectors / cell $=6$, sector reuse factor $=1$ ). 76

4.8 Scheme 133 (cell reuse factor $=1$, sectors $/$ cell $=3$, sector reuse factor $=3$ ). 77

4.9 Scheme 163 (cell reuse factor $=1$, sectors $/$ cell $=6$, sector reuse factor $=3$ ). 78

4.10 Scheme 166 (cell reuse factor $=1$, sectors $/$ cell $=6$, sector reuse factor $=6$ ). 79

4.11 Scheme 311 (cell reuse factor $=3$, sectors $/$ cell $=1$, sector reuse factor $=1$ ). 80

4.12 Scheme 331 (cell reuse factor $=3$, sectors $/$ cell $=3$, sector reuse factor $=1$ ). 82

4.13 Scheme 333 (cell reuse factor $=3$, sectors $/$ cell $=3$, sector reuse factor $=3$ ). 83

4.14 Scheme 361 (cell reuse factor $=3$, sectors $/$ cell $=6$, sector reuse factor $=1$ ). 84

4.15 Scheme 363 (cell reuse factor $=3$, sectors $/$ cell $=6$, sector reuse factor $=3$ ). 86

4.16 Scheme 366 (cell reuse factor $=3$, sectors $/$ cell $=6$, sector reuse factor $=6$ ). 87

$4.17 \mathrm{CDF}$-throughput for cell frequency reuse $=1 \ldots \ldots . \ldots 89$

$4.18 \mathrm{CDF}$-throughput for cell frequency reuse $=3 \ldots \ldots \ldots$

4.19 CDF of normalized throughput for Scheme 163. . . . . . . . . 92 


\section{List of Symbols}

$\begin{array}{ll}\underline{a}^{(s)} & \text { message word of transmitter } s \\ \underline{\hat{a}}^{(s)} & \text { estimation of source symbols of transmitter } s \\ a_{n}^{(s)} & n \text {th bit of message word of transmitter } s \\ A_{\text {max }} & \text { maximum power attenuation of transmit antenna in dB } \\ b_{n}^{(s)} & \text { anti-biasing term of transmitter } s \\ \underline{c}^{(s)} & \text { code word of transmitter } s \\ c_{n}^{(s)} & n \text {th bit of code word of transmitter } s \\ C_{R} & \text { connection rate in bits per channel use } \\ \underline{d}^{(s)} & \text { interleaved code word of transmitter } s \\ d_{n}^{(s)} & n \text {th interleaved symbol of interleaved code word of transmitter } s \\ G_{r} & \text { antenna gain of MS receive antenna in dBi } \\ G_{t}(\theta) & \text { antenna gain of BS transmit antenna in dB } \\ h_{c, i} & \text { complex channel gain of the } i \text { th ISI path } \\ h_{c, i}^{(s, a)} & \text { complex channel gain from transmitter } s \text { to antenna } a \\ h_{l} & \text { impulse response of discretetime complex lowpass equivalent channel } \\ h_{l}^{(s, a)} & \text { impulse response from transmitter } s \text { to antenna } a \\ h_{T}(t) & \text { impulse response of transmitted pulse-shaping filter (continuous) } \\ h_{T R}(t) & \text { combined impulse response of transmit (pulse-shaping) and receive } \\ H_{k}^{(s, a)} & \text { (matched) filters (continuous) } \\ H_{T}(f) & \text { DFT of impulse response between transmitter } s \text { and antenna } a \\ J^{(s)} & \text { frequency response of transmitted pulse-shaping filter } \\ L & \text { mean square error between } z_{n}^{(s)} \text { and } v_{n}^{(s)} \\ M & \text { ISI delay spread in symbols } \\ n_{d} & \text { number of points in signal constellation } \\ N & \text { number of bits in one interleaved symbol } \\ N_{0} & \text { number of symbols in one interleaved code word } \\ N_{a} & \text { single-sided noise power spectral density } \\ N_{c} & \text { number of bits in one message word } \\ & \end{array}$




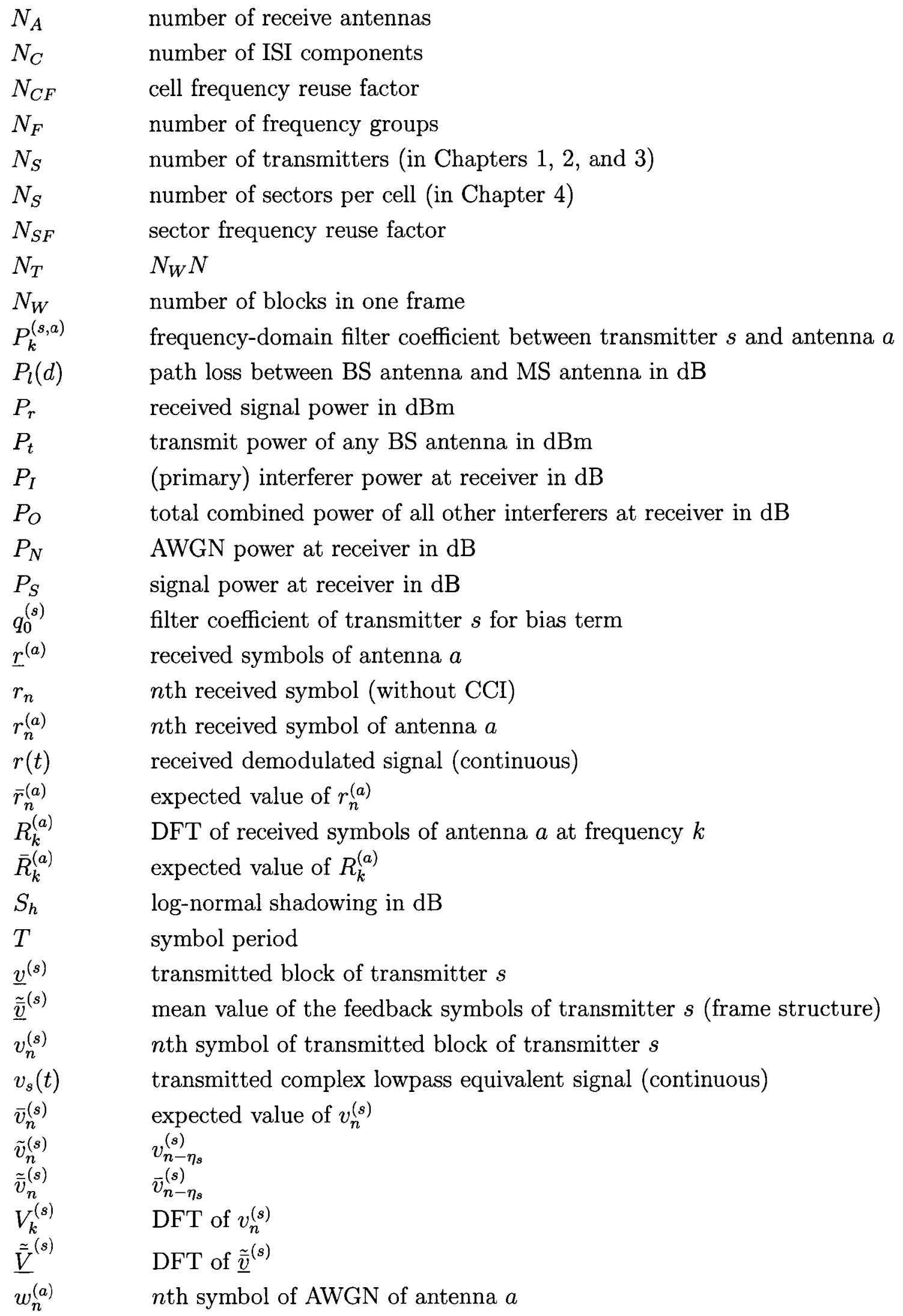


$W_{k}^{(a)}$

$\underline{z}^{(s)}$

$\underline{\tilde{z}}^{(s)}$

$z_{n}^{(s)}$

$\bar{z}_{n}^{(s)}$

$\tilde{z}_{n}^{(s)}$

$\alpha_{s}$

$\beta$

$\Delta$

$\eta$

$\eta_{\text {nom }}$

$\eta_{s}$

$\gamma_{n}^{(s)}(d)$

$\Gamma$

$\mu_{n}^{(s)}(d)$

$\phi_{n}^{(s)}(d)$

$\psi_{n}^{(s)}(c)$

$\rho$

$\sigma_{c, i}^{2}$

$\sigma_{v, n, s}^{2}$

$\sigma_{s}$

$\bar{\sigma}_{v, s}^{2}$

$\bar{\sigma}_{z, s}^{2}$

$\tau_{c, i}$

$\tau_{c, i}^{(s, a)}$

$\bar{\tau}_{c, i}$

$\theta_{3 \mathrm{~dB}}$

DFT of $w_{n}^{(a)}$

detector output for transmitter $s$ (block structure)

detector output for transmitter $s$ (frame structure)

$n$th symbol of detector output for transmitter $s$ (block structure)

expected value of $z_{n}^{(s)}$

$n$th symbol of detector output for transmitter $s$ (frame structure)

large-scale signal attenuation due to path loss and shadowing

rolloff factor of pulse-shaping filter

power discrepancy between the signal and the interference in $\mathrm{dB}$

normalized throughput

nominal throughput in message bits per channel use

coarse propagation delay (in symbols) of the signal from transmitter $s$

metric value of transmitter $s$ at time $n$ conditioned on $d$

$N_{A} \times N_{A}$ matrix

mean of $z_{n}^{(s)}$ conditioned on $d$

interleaved soft feedback symbol of transmitter $s$ at time $n$

soft feedback symbol of transmitter $s$ at time $n$

path loss exponent

$\mathcal{E}\left[\left|h_{c, i}\right|^{2}\right]$

variance of the $n$-th transmitted symbols by transmitter $s$

standard deviation of log-normal shadowing

time-average variance of all transmitted symbols by transmitter $s$

time-average variance of $z_{n}^{(s)}$ conditioned on $d$

propagation delay of the $i$ th ISI path

propagation delay between transmitter $s$ and antenna $a$

average propagation delay of the $i$ th ISI path

$3 \mathrm{~dB}$ beamwidth of transmit antenna in degrees 


\section{List of Acronyms}

$\begin{array}{ll}\text { AWGN } & \text { Additive White Gaussian Noise } \\ \text { BER } & \text { Bit Error Rate } \\ \text { BS } & \text { Base Station } \\ \text { CCI } & \text { Co-Channel Interference } \\ \text { CDF } & \text { Cumulative Distribution Function } \\ \text { CRC } & \text { cyclic redundancy check } \\ \text { dB } & \text { Decibel } \\ \text { dBi } & \text { Decibel Isotropic } \\ \text { dBm } & \text { Power Level in Decibels Relative to 1mW } \\ \text { DFE } & \text { Decision Feedback Equalization } \\ \text { DFT } & \text { Discrete Fourier Transform } \\ \text { FDE } & \text { Frequency Domain Equalization } \\ \text { FFT } & \text { Fast Fourier Transform } \\ \text { IC } & \text { Interference Canceler } \\ \text { IFFT } & \text { Inverse Fast Fourier Transform } \\ \text { ISI } & \text { Inter-Symbol Interference } \\ \text { LE } & \text { Linear Equalization } \\ \text { MAP } & \text { Maximum a posteriori Probability } \\ \text { MCU } & \text { Metric Calculation Unit } \\ \text { MIMO } & \text { Multiple-Input Multiple-Output } \\ \text { MLSE } & \text { Maximum Likelihood Sequence Equalization } \\ \text { MMSE } & \text { Minimum Mean Square Error } \\ \text { MS } & \text { Mobile Station } \\ \text { OFDM } & \text { Orthogonal Frequency Division Multiplexing } \\ \text { QPSK } & \text { Quadrate Phase Shift Keying } \\ \text { SDMA } & \text { Space Division Multiple Access } \\ \text { SISO } & \text { Soft-Input Soft-Output } \\ \text { SINR } & \text { Signal to Interferer-plus-Noise Ratio } \\ \text { SIR } & \text { Signal-to-Interference Ratio } \\ & \end{array}$


SNR Signal-to-Noise Ratio

TDMA Time Division Multiple Access

WER Word Error Rate

xii 


\section{Acknowledgment}

I would like to express my sincere gratitude to my thesis supervisor, Dr. Ian Marsland, for his great help, support, understanding and supervising this research work. His patience and extensive guidance helped me fulfill this thesis smoothly. I learned much more from this research than I had expected.

Sincere thanks to the Department of Systems and Computer Engineering, Dr. Ian Marsland and Dr. Halim Yanikomeroglu for the financial support during my studies. Without their generosity, my studies could not be completed.

Thanks to Dr. Peter Galko, Dr. Thomas Kunz, Dr. Florence Danilo-Lemoine, Dr. Halim Yanikomeroglu, Dr. Richard M. Dansereau, Dr. Sergey Loyka, and Dr. Claude D'Amours, who provided the fundamental knowledge for my research.

Thanks to Jianhong Fang, Haoming Li and Yaobin Wen, who provided helps during my studies.

Finally, I acknowledge the constant support and encouragement from my family throughout my studies. 


\section{Chapter 1}

\section{Introduction}

Co-channel interference (CCI), resulting from the reuse of the available network resources (e.g., frequency, time) by several users, is a major limiting factor on the capacity in wireless communication systems. In addition, multi-path propagation, created by multiple signal paths between the transmitter and the receiver due to the reflection of the transmitted signal by physical obstacles, is another significant issue. Inter-symbol interference (ISI), caused by the different versions of the multi-path signal arriving at the receiver at different times, has a critical impact on communication link quality.

Generally speaking, any received data in a reuse system can be divided into four components: the desired data, CCI from other transmitters, ISI from neighboring symbols, and additive white Gaussian noise (AWGN). In this thesis, an asynchronous frequency-domain iterative multiple-input multiple output (MIMO) detector is implemented to suppress CCI and ISI. 


\subsection{Review of CCI Mitigation}

In recent years, the demand for high speed wireless applications, requiring considerable radio frequency resources, has increased dramatically. Meanwhile, the available radio frequency spectrum is becoming scarce, so efficient use of the spectrum becomes necessary.

To achieve efficient spectrum usage, the available frequency band for a wireless system, such as a cellular system, is traditionally divided into several frequency groups, each of which is allocated to cells or base stations (BS) in a pattern such that any two base stations using the same frequency group are kept as far away as possible. Thus the frequency used within one cell can be re-used in other cells. The frequency reuse factor is introduced to express how many frequency groups are used in a system. However, the frequency reuse results in co-channel interference (CCI). A smaller frequency reuse factor can achieve better spectral efficiency but produce more CCI; a larger factor obtains worse spectral efficiency but causes less CCI. If the CCI can be effectively eliminated at the receiver, a smaller frequency reuse factor can be used for higher spectral efficiency [1][2]. Figure 1.1 shows two CCI examples caused by frequency reuse. As illustrated, when the reuse factor is three the interfering signals must travel a much greater distance (and will therefore likely be highly attenuated) than the desired signal, so the CCI will have less of an adverse effect than the case when the reuse factor is one.

Similar to the frequency reuse in cells, cell sectoring is a way to improve the spectral efficiency. Cell sectoring divides a hexagonal cell into several pie-shaped 
freq. reuse factor $=1$

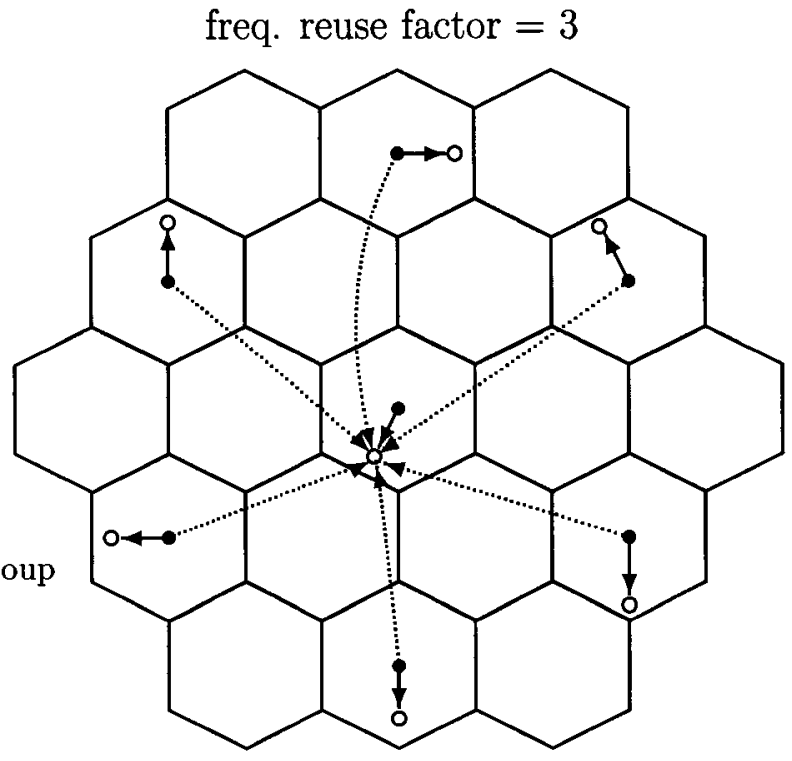

Figure 1.1: CCI examples.

sectors, each of which has a directional antenna and a (possibly) different frequency group thus introducing less CCI than the original omni-directional antenna approach. Two types of sectoring are commonly used in practice. They are 120-degree sectoring, in which a cell is evenly divided into 3 sectors, and 60-degree sectoring, in which a cell is divided into 6 sectors. Since cell sectoring confines a frequency group to a smaller area (a sector rather than a cell), a smaller frequency cell reuse factor can be deployed to improve spectral efficiency. The disadvantage of cell sectoring is the increased number of handoffs [1][2].

Other schemes, such as cell splitting and cell sizing (microcellular networks), can also improve spectrum efficiency and reduce CCI [1][2] but are more expensive as additional base stations are required.

The schemes mentioned above, such as frequency reuse, cell sectoring, cell splitting and cell sizing, can reduce the CCI from the network design point of view. However, 
as long as the frequency groups are re-used within a wireless network, CCI always exists.

\subsection{Review of ISI Elimination}

To compensate or reduce the ISI in the received signal, researchers have introduced a method called equalization. Several equalization methods will be reviewed in this section. They are linear equalization (LE), decision feedback equalization (DFE), maximum likelihood sequence equalization (MLSE), maximum a posteriori probability (MAP) equalization, and turbo equalization.

A linear equalizer is usually a transversal filter with a number of taps whose weight coefficients can be adjusted to suppress ISI while maximizing the signal-to-noise ratio (SNR). For channels with spectral nulls, linear equalization performs poorly because the compensation for the spectral nulls enhances the additive noise [3].

A decision feedback equalizer is composed of a feedforward filter and a feedback filter. The feedforward filter is similar to the linear equalizer. The feedback filter takes the decisions of previously detected symbols as its input such that it can remove the section of the ISI caused by the previously detected symbols. Compared to linear equalization, decision feedback equalization can achieve a considerable gain [3].

With MLSE the channel is modeled as a finite-state machine, and the Viterbi algorithm is used to determine the most likely path through the corresponding trellis. Although MLSE is optimal from a probability of error viewpoint, the computational complexity of MLSE grows exponentially with the ISI span, which makes it not ap- 


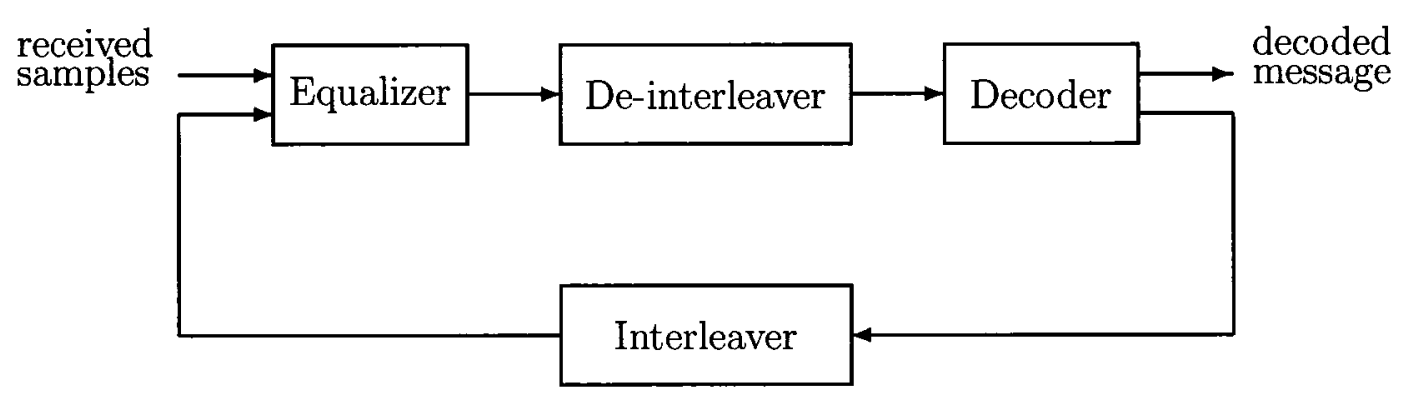

Figure 1.2: Block diagram of a turbo equalizer

plicable for most practical channels. Furthermore, the hard output of the Viterbi algorithm makes it a poor choice when used with channel codes [3].

MAP equalization, with decisions made on a symbol-by-symbol basis, can provide soft output to the channel decoder [4]. Similar to MLSE, MAP equalization is also not suitable for the large ISI delay.

Turbo equalization was proposed by C. Douillard et al in 1995 [5]. As shown in Figure 1.2, a turbo equalizer consists of an equalizer, a decoder, and a de-interleaver and interleaver pair, working in an iterative way. For the first iteration, the equalizer does not have any knowledge of the transmitted symbols. The output is sent through the de-interleaver to the decoder. After decoding the channel code, the estimated transmitted data are fed back through the interleaver to the equalizer for the second iteration. This feedback is used by the equalizer in the second (and subsequent) iterations as a priori knowledge of the transmitted data to better estimate the ISI. After several iterations, the estimated data are more reliable and the output bit error rate (BER) decreases. However, due to the deployment of the MAP decoder, the computational complexity in [5] is high. 
A modified turbo equalizer, based on an interference canceler (IC) instead of a MAP equalizer, was proposed by A. Glavieux et al in 1997 [6]. An IC consists of a matched filter which maximizes the SNR but also elongates the ISI. From the output of the matched filter, a locally generated estimate of the ISI is subtracted. This ISI estimate is generated based on knowledge of the other symbols in the transmitted block, as determined by the channel decoder on the previous iteration. For the first iteration, when no such knowledge is available, a LE is used instead of the IC. The resulting turbo equalizer works quite well, much better than non-iterative equalizers, but not nearly as well as MAP-based turbo equalization. The IC, however, has much lower complexity than the MAP equalizer, with complexity growing linearly with ISI spread.

The main weakness with IC-based turbo equalization is its bimodal operation. It operates under the assumption that it either has no knowledge of the other transmitted symbols (as on the first iteration) or perfect knowledge (on the second and subsequent iterations). In practice, the reliability of the decisions, while better than nothing, are far from perfect, so the IC is only really effective at high SNRs.

To overcome this weakness, M. Tüchler et al proposed the minimum mean square error (MMSE)-based soft-input / soft-output (SISO) equalizer in 2000 [7][8]. This equalizer adjusts the filter taps dynamically depending on the reliability of the data from the previous iteration, so the filter is able to operate in a continuous range of conditions, from a LE (no knowledge) to an IC (perfect knowledge) at the extremes. Simulation results confirm that the turbo equalizer based on this approach is nearly as effective as MAP-based turbo equalization, with complexity similar to that of 
IC-based turbo equalization.

Both the IC and the MMSE-based SISO equalizer use time-domain linear filtering, with computational complexity that grows linearly with the ISI spread. However, for highly dispersive channels, even this complexity can be too great. To overcome this limitation, several researchers proposed frequency-domain equalization (FDE) [9][10]. The computational complexity of the FDE is essentially independent of the ISI spread. In 2000, M. Tüchler et al developed the algorithm for frequency-domain turbo equalization [11][12]. Thus the advantages of frequency-domain processing and the turbo iterative method are combined.

\subsection{Multi-user Detection for ISI Channels}

In recent years, the use of multiple-input / multiple-output (MIMO) channels has received considerable attention [13][14]. By employing multiple transmit and receive antennas, it is possible to significantly increase the channel capacity and exploit spatial diversity to reduce the effect of multi-path fading. Some of the techniques for MIMO channels can also be used to implement space division multiple access (SDMA).

As shown in Figure 1.3, in the uplink of a SDMA system, multiple users simultaneously transmit data to a base station within a single frequency band. Equipped with multiple receive antennas, the base station uses sophisticated signal processing techniques to isolate, detect and decode the signal from each user (multi-user detection). 


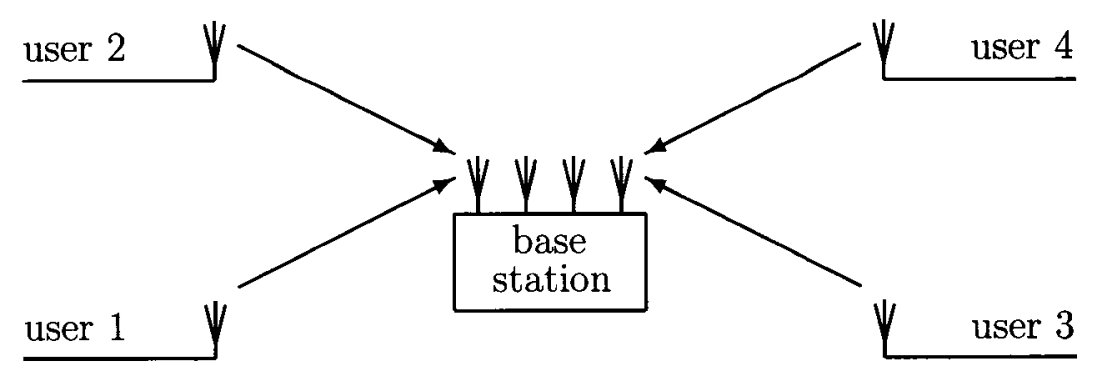

Figure 1.3: Uplink of a SDMA system.

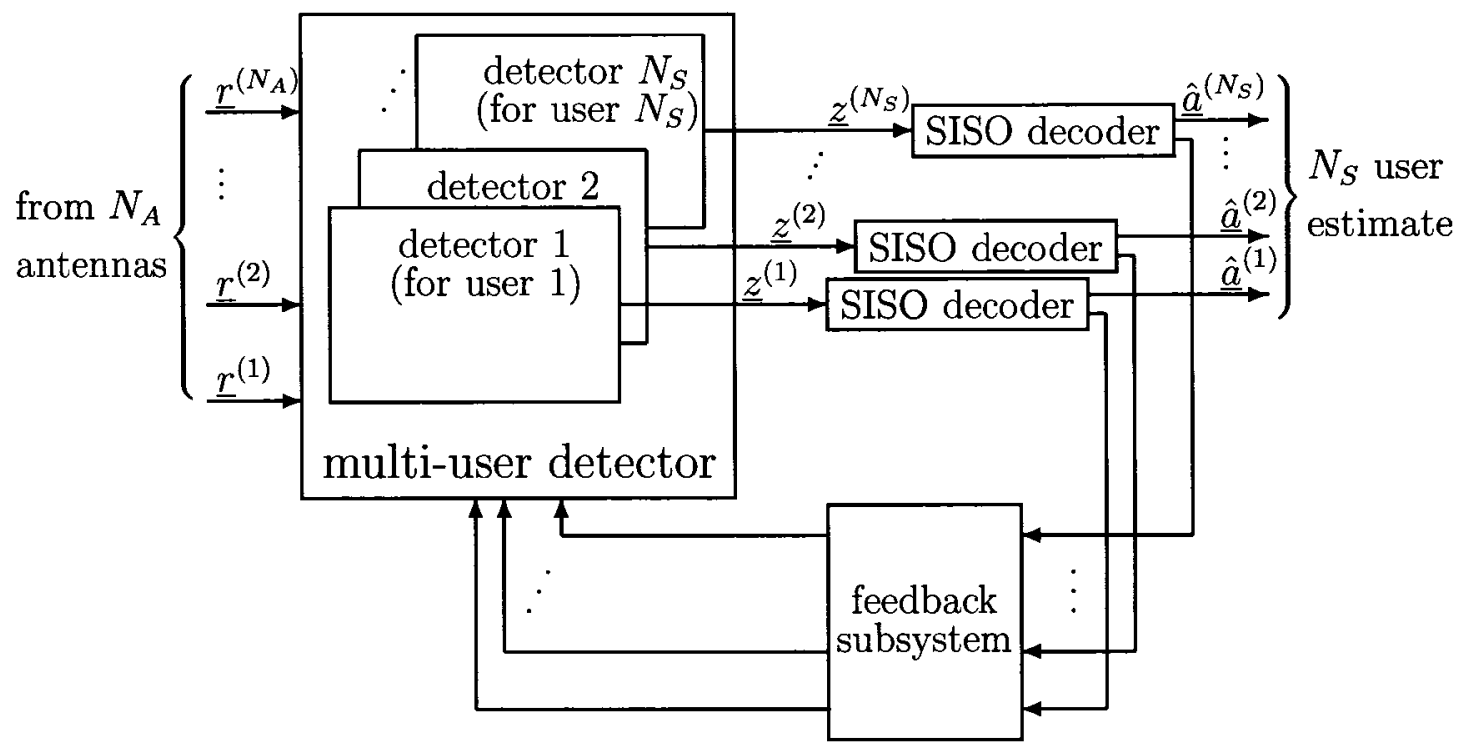

Figure 1.4: Block diagram of an iterative multi-user detector.

In 2003, T. Abe et al [15] proposed an iterative time-domain multi-user detector / equalizer [15], based on the MMSE-based SISO equalizer. Figure 1.4 shows a block diagram for this iterative multi-user detector, with $N_{A}$ receive antennas and the ability to support $N_{S}$ different users. When the signals from the $N_{S}$ transmit antennas are received by the $N_{A}$ receive antennas, $N_{S}$ detectors filter these signals and pass them to $N_{S}$ SISO channel code decoders respectively. The estimated versions of the transmitted signals are fed back to the decoders to improve the signal detection performance for the next iteration. After several iterations, the final estimates of the 
transmitted data can be output. Though T. Abe's algorithm is very effective in multiuser detection and combating ISI, the computational complexity of his time-domain model makes this algorithm inappropriate for the large ISI spread case.

In her Master's thesis, J. Lin re-derived T. Abe's algorithm in the frequency domain [16]. The overall structure of J. Lin's model is identical to that of T. Abe's model, except that all signals are processed in the frequency domain. Therefore, J. Lin's model is more computational effective, especially for channels with large ISI spread.

\subsection{Thesis Contributions}

Recognizing the similarities between SDMA and cellular system frequency reuse, in this thesis we explore the possibility of applying multi-user detection techniques to the problem of CCI suppression. Instead of having multiple users within a single cell trying to simultaneously transmit to a base station, in our case we have multiple base stations transmitting simultaneously. Although the transmitted signals are intended for different users, any particular user will receive all of them, but all the signals other than the one from that user's serving base station will be CCI. Nonetheless, an effective multi-user detector should, in some situations, be able to distinguish between these signals and extract the desired signal from the CCI. In particular, we consider a modified version of the frequency-domain multi-user detector / equalizer proposed by J. Lin [16].

This approach to suppressing CCI is shown to be very effective, with near-perfect 
suppression possible, provided that the SNR of the desired signal is sufficiently large and there is only a small number of strong interferers. The number of interferers that can be canceled depends on the number of antennas at the receiver. Since we are focusing on a downlink environment, and acknowledging that physical constraints may limit the number of antennas on a portable receiver, we only consider devices with one or two receive antennas, so only one or two interferers can be canceled. Furthermore, the system is much more adept at canceling strong interferers (with signal levels similar to the desired signal) than weak ones, but fortunately uncanceled weak interferers are less of an impediment to reliable communication than uncanceled strong ones.

An important difference between the multi-user detection problem and the CCI suppression problem involves the synchronicity of the signals. Whereas in the multiuser detection scenario the base station may be able to control the users so that their signals arrive at roughly the same time (synchronous signal reception), this is not realistic in the CCI scenario. Even if the base stations all transmit synchronously, the signals at the receivers will be asynchronous due to the wide variations among the propagation distances.

The algorithm derived by J. Lin is only applicable to the fully synchronous case. All data blocks from the different users have to arrive at the receiver at the same time. For example, the $i$ th symbol of a block from any user must reach the receiver at the exact same time as when the $i$ th symbol of a block from every other user reaches it.

In this thesis, we generalize J. Lin's model to be applicable to the asynchronous case. The overall structure of our model is still the same as that of J. Lin's model 
(Figure 1.4). However, the detectors of our model are able to handle arbitrary symbol offsets among users. Other minor modifications have also been done to make the model more practical.

In particular,

1. We introduce the frequency-domain turbo detection / equalization for asynchronous CCI suppression.

2. We simulate the model with a $\mathrm{C}++$-based simulator. The simulation results show that our model can cancel the ISI and the CCI. Compared to the synchronous case, the penalty for the asynchronous case is small.

3. A three-tier cellular simulation model is used to show the improvement in throughput that the CCI suppression can provide.

\subsection{Thesis Organization}

Chapter 2 presents a description of the asynchronous system model and describes the modifications to J. Lin's system. Chapter 3 studies the asynchronous system performance over three different ISI channels. Chapter 4 uses a 3-tier cellular model to demonstrate the improvements in system throughput that can be realized by using the CCI suppressor. Chapter 5 presents the conclusions and the future work. The Appendix contains a derivation of the mathematical formulae used to calculate the filter coefficients. 


\section{Chapter 2}

\section{System Model Description}

As Figure 2.1 shows us, our system model consists of $N_{S}$ transmitters (one desired and $N_{S}-1$ interferers), a multiple-input multiple-output (MIMO) channel, and an iterative receiver which has $N_{A}$ antennas and a muti-user detector / decoder. Since the multi-user detector is used for CCI elimination, the $N_{S}-1$ CCI outputs are omitted.

In our model, each of the $N_{S}$ transmitters has only one antenna. The data to be transmitted are first encoded by a convolutional encoder. Then the encoded data

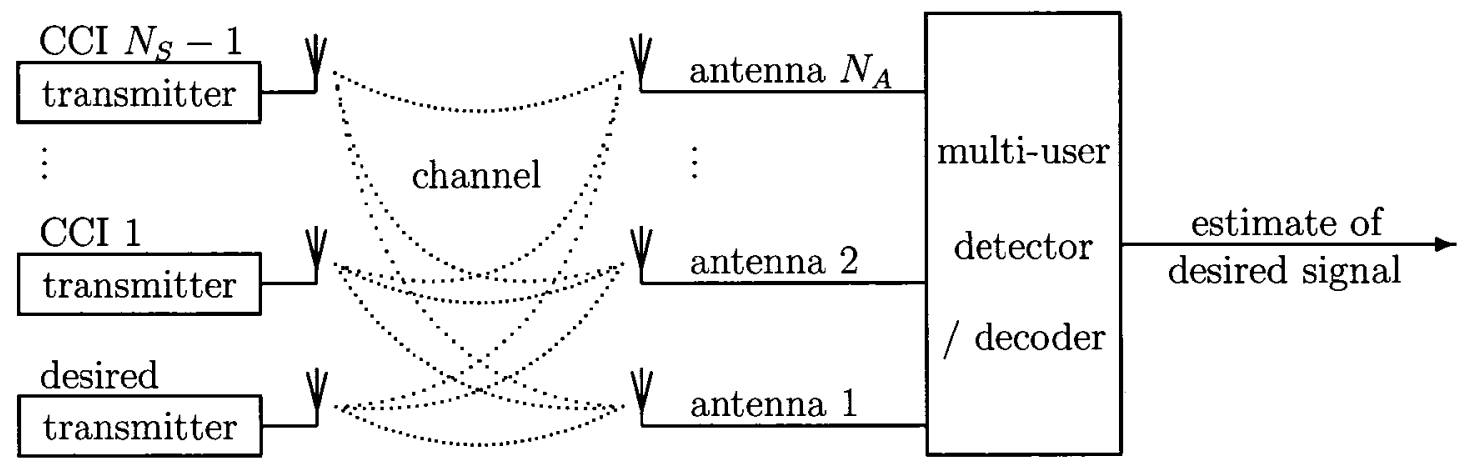

Figure 2.1: Block diagram of the MIMO system model. 


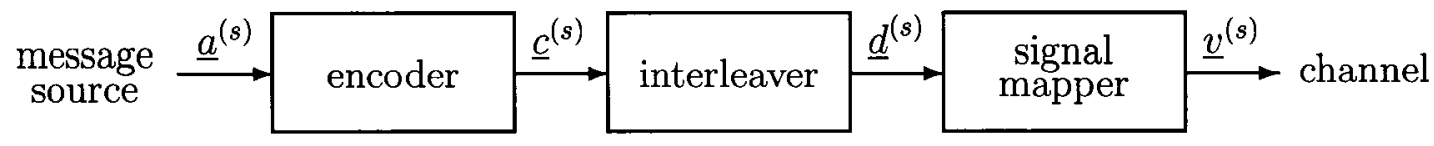

Figure 2.2: Block diagram of a transmitter.

are passed to an interleaver and a signal mapper. The modulated signal is sent to the channel through the antenna. Synchronization among the $N_{S}$ transmitters is not required.

The modulated signals experience frequency selective multi-path fading in the channel, resulting in inter-symbol interference (ISI). The desired signal also suffers from the interference caused by the other transmitters who share the same channel, resulting in co-channel interference (CCI). Furthermore, additive white Gaussian noise (AWGN) is added in this channel. Due to different propagation distances, the average signal power of the user and CCI's may differ at the receiver.

After being received by the $N_{A}$ receive antennas, the received signals are sent to $N_{S}$ detectors followed by decoders. The $N_{S}$ soft-decoded estimates are then fed-back to the detectors for better estimations. Figure 1.4 shows the structure of the iterative receiver.

\subsection{Transmitter}

The block diagram of a transmitter is shown in Figure 2.2. The message source, the encoder, the interleaver and the signal mapper will be presented in the following.

For any transmitter, one message word consists of $N_{a}$ message bits. Each message bit is independent of the other bits within the same message word, and the messages 
from each transmitter are independent of those from the other transmitters. Let $\underline{a}^{(s)}=\left[a_{0}^{(s)}, a_{1}^{(s)}, \ldots, a_{N_{a}-1}^{(s)}\right]$ denote the message word from transmitter $s$, and $a_{n}^{(s)}$ denote the $n$th bit of that word.

The message words are sent to the encoder, where a convolutional code is applied for error correction. The encoder works on a word-by-word basis, with the trellis terminated after each word has been encoded. After encoding, a message word turns into a code word with $N_{c}$ code bits. The corresponding code word is $\underline{c}^{(s)}=\left[c_{0}^{(s)}, c_{1}^{(s)}, \ldots, c_{N_{c}-1}^{(s)}\right]$

The code words are passed to an interleaver, which also processes data on a wordby-word basis. The interleaver is used to permute the encoded data within a code word such that adjacent interleaved bits are less correlated. The interleaved bits of each word are grouped into $N$ symbols, each of which has $n_{d}$ bits, where $n_{d}$ depends on the modulation scheme $\left(n_{d}\right.$ is the number of bits transmitted in each modulation symbol). Therefore, we have $N \times n_{d}=N_{c}$. The interleaved code word is denoted as $\underline{d}^{(s)}=\left[d_{0}^{(s)}, d_{1}^{(s)}, \ldots, d_{N-1}^{(s)}\right]$.

The interleaved code words are then passed to a signal mapper, where interleaved symbols are mapped to modulation symbols according to the $M$-ary signal constellation $\left(M=2^{n_{d}}\right)$. The block of symbols corresponding to one message word is herein referred to as a symbol block, and is denoted by $\underline{v}^{(s)}=\left[v_{0}^{(s)}, v_{1}^{(s)}, \ldots, v_{N-1}^{(s)}\right]$. The mapping is defined as $v_{n}^{(s)}=\operatorname{SM}\left[d_{n}^{(s)}\right]$, where $\operatorname{SM}[\cdot]$ is the mapping function between digital values and constellation points. The modulation symbols in the symbol block are sent to the transmit antenna and over the channel. 


\subsection{Channel Model}

The discrete-time channel model includes the pulse-shaping filter and the modulator at the transmitter, the analog multi-path fading channel, and the de-modulator, the receive filter and the symbol-rate sampler at the receiver.

The transmitted complex lowpass equivalent signal is

$$
v_{s}(t)=\sum_{n} v_{n}^{(s)} h_{T}(t-n T)
$$

where $h_{T}(t)$ is the transmitted pulse shape and $T$ is the symbol period. For the simulation results in this thesis we consider the case where $h_{T}(t)$ is the impulse response of a filter with a root raised-cosine frequency response

$$
H_{T}(f)= \begin{cases}\sqrt{T} & 0 \leq|f|<\frac{1-\beta}{2 T} \\ \sqrt{T} \cos \left[\frac{\pi T}{2 \beta}\left(|f|-\frac{1-\beta}{2 T}\right)\right] & \frac{1-\beta}{2 T} \leq|f|<\frac{1+\beta}{2 T} \\ 0 & |f| \geq \frac{1+\beta}{2 T}\end{cases}
$$

where $\beta$ is the filter's rolloff factor.

For a single link between one transmit antenna and one receive antenna, we consider a frequency selective slow Rayleigh fading channel model. This model consists of $N_{C}$ independent multi-path components, and the received demodulated signal (without CCI or AWGN) is given by

$$
r(t)=\sum_{i=0}^{N_{C}-1} h_{c, i} v_{s}\left(t-\tau_{c, i}\right)
$$

where $h_{c, i}$ is the complex channel gain of the $i$ th path, and $\tau_{c, i}$ is its propagation delay. $h_{c, i}$ is modeled as a zero-mean circularly symmetric complex Gaussian random variable, with variance $\sigma_{c, i}^{2}=\mathcal{E}\left[\left|h_{c, i}\right|^{2}\right]$. To better reflect the asynchronous 


\begin{tabular}{|l|l|c|c|c|}
\hline \multicolumn{2}{|l|}{$i$} & 0 & 1 & 2 \\
\hline \multicolumn{2}{|l|}{$\bar{\tau}_{c, i}(\mu \mathrm{s})$} & 0 & 4 & 10 \\
\hline \multirow{2}{*}{$\sigma_{c, i}^{2}$} & $(\mathrm{~dB})$ & 0 & -5 & -10 \\
\cline { 2 - 5 } & (normalized) & 0.706 & 0.223 & 0.071 \\
\hline
\end{tabular}

Table 2.1: Power delay profile of SUI-5 channel.

nature of our system, $\tau_{c, i}$ is modeled as a uniformly distributed random variable over $\left[\bar{\tau}_{c, i}-T / 2, \bar{\tau}_{c, i}+T / 2\right)$, where $\bar{\tau}_{c, i}$ is the average delay of the path. Both $\sigma_{c, i}^{2}$ and $\bar{\tau}_{c, i}$, along with $N_{C}$, describe the power delay profile of the particular channel model of interest. For example, for the SUI-5 channel model [17], we have $N_{C}=3$ and the parameters are listed in Table 2.1. Note that the variances are normalized so that their sum is unity.

After filtering with a matched filter (matched to $h_{T}(t)$ ), the signal is sampled at the symbol rate. The received samples (again, without CCI or AWGN) are given by

$$
r_{n}=\sum_{l=0}^{L-1} h_{l} v_{n-l}
$$

where $\left\{v_{n}\right\}$ are the transmitted symbols, and $h_{l}$ is the impulse response of the discretetime complex lowpass equivalent channel. These are given by

$$
h_{l}=\sum_{i=0}^{N_{C}-1} h_{c, i} h_{T R}\left(l T-\tau_{c, i}\right),
$$

where $h_{T R}(t)$ is the combined impulse response of the transmit (pulse-shaping) and receive (matched) filters. The delay spread of the channel is $L$ samples, where $L-1$ is the largest value of $l$ such that $h_{l}$ is non-negligible.

Because multiple base stations are transmitting simultaneously, the simple channel model of Equation 2.4 is extended to reflect the CCI. The received samples on antenna 
$a$ are given by

$$
r_{n}^{(a)}=\sum_{s=1}^{N_{S}} \sum_{l=0}^{L-1} h_{l}^{(s, a)} v_{n-l-\eta_{s}}^{(s)}+w_{n}^{(a)},
$$

where $\eta_{s}$ is the coarse propagation delay (in samples) of the signal from transmitter $s$, $w_{n}^{(a)}$ is the AWGN, which is modeled as a stationery white Gaussian random process, with zero mean and a single sided power spectral density of $N_{0}$, so $\mathcal{E}\left[\left|w_{n}^{(a)}\right|^{2}\right]=N_{0}$, and $h_{l}^{(s, a)}$ is the impulse response of the channel from transmitter $s$ to antenna $a$, which is given by

$$
h_{l}^{(s, a)}=\alpha_{s} \sum_{i=0}^{N_{C}-1} h_{c, i}^{(s, a)} h_{T R}\left(l T-\tau_{c, i}^{(s, a)}\right)
$$

where $h_{c, i}^{(s, a)}$ and $\tau_{c, i}^{(s, a)}$ are the complex channel gains (Rayleigh fading) and fine-scale propagation delays of the multi-path components, and $\alpha_{s}$ is the large-scale signal attenuation due to path loss and shadowing. Although $h_{c, i}^{(s, a)}$ and $\tau_{c, i}^{(s, a)}$ are different for each $(s, a)$ link, they are randomly distributed according to the same power delay profile. The large-scale signal attenuation, $\alpha_{s}$, differs for each transmitter but is the same for each receive antenna. This is because the receive antennas are close together but the large-scale signal attenuation varies slowly over distance (compared to the Rayleigh fading). Similarly, the coarse propagation delay, $\eta_{s}$, is also different for each transmitter (because the distances from the transmitters are all different), but the same for each receive antenna. Discrepancy between the propagation delays between the receive antennas, and fine resolution of the delays (because the delay is unlikely to be an even multiple of the symbol period) are incorporated into $h_{l}^{(s, a)}$ by the random nature of $\tau_{c, i}^{(s, a)}$. Note that $\eta_{s}$ could also be incorporated into $\tau_{c, i}^{(s, a)}$, but it is convenient to keep it separate to facilitate the design of the receiver. 


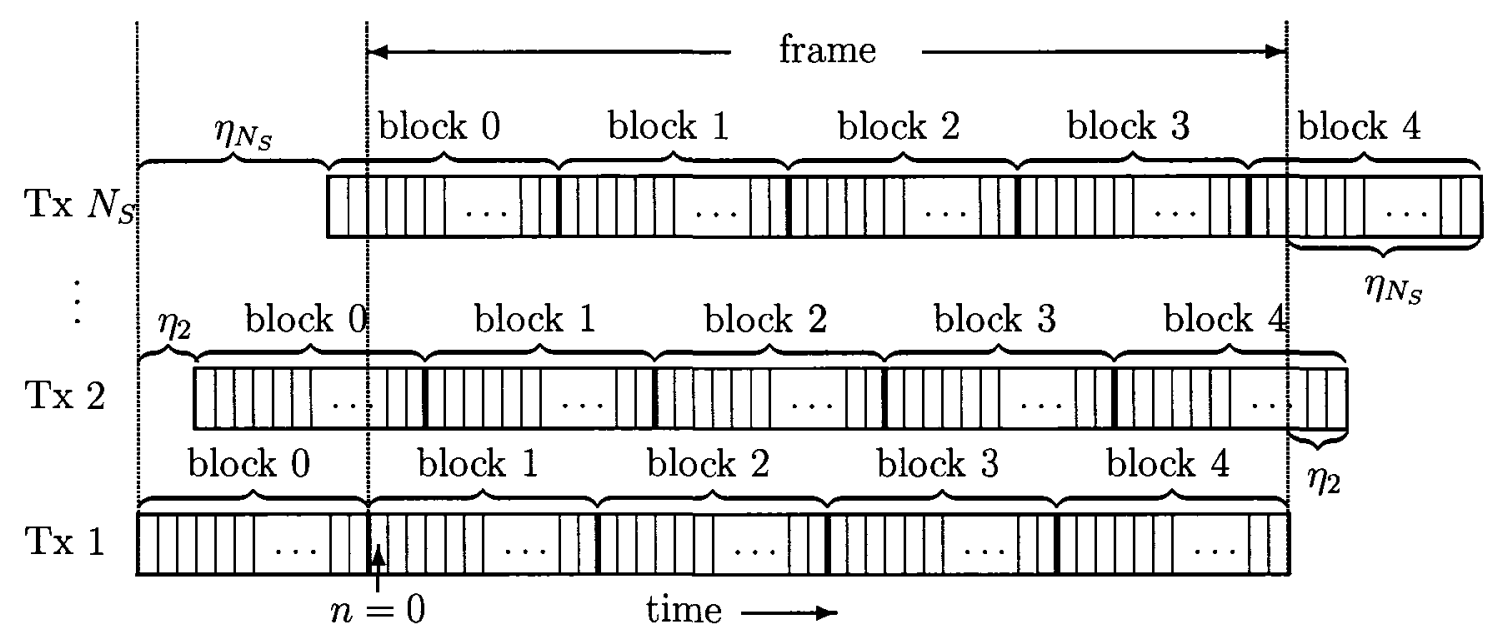

Figure 2.3: Asynchronous block reception.

We consider a continuous transmission system, where each transmitter continuously transmits symbol blocks, one block after another. For the sake of discussion, and without loss of generality, we assume that the receivers all transmit synchronously ${ }^{1}$. That is, they all begin transmitting a symbol block at the exact same time. However, because of the different propagation delays, $\eta_{s}$, the blocks are received asynchronously. We denote the block that begins transmission at time $n=0$ as Block \#1. Using this notation, we note that $v_{n-l-\eta_{s}}^{(s)}$ in Equation 2.6 refers to a symbol in a previous block when $n-l-\eta_{s}$ is less than 0 , and in a subsequent block when $n-l-\eta_{s} \geq N$. For arbitrary index $m=n-l-\eta_{s}$, the block number is $\lfloor m / N\rfloor+1$, where $\lfloor\cdot\rfloor$ denotes the "floor" function, and the offset of the index into the block is $m-\lfloor m / N\rfloor N$. 


\subsection{Receiver}

Because of the asynchronous nature of our channel model, each desired symbol block is interfered by parts of two different symbol blocks from each other transmitter, as illustrated in Figure 2.3 (with $\eta_{1}=0$ ). Furthermore, because there are no guard bands between blocks from the same transmitter, the time dispersive channel links cause inter-block interference. J. Lin used cyclic prefixes between the blocks (like the cyclic prefixes used in OFDM systems) to prevent the inter-block interference, but her receiver was unable to cope with asynchronous block reception.

Our proposed receiver is very similar to J. Lin's, except that instead of processing samples on a block-by-block basis, our receiver processes samples in frames, with each frame spanning $N_{W}$ blocks, so each frame contains $N_{T}=N_{W} N$ received samples. The received frame from receive antenna $a$ is $\underline{r}^{(a)}=\left[r_{0}^{(a)}, r_{1}^{(a)}, \ldots, r_{N_{T}-1}^{(a)}\right]$ where $r_{n}^{(a)}$ is given by Equation 2.6. We assume the receiver is able to maintain synchronization with the desired signal, so it begins sampling for the frame with the first sample containing the first symbol of Block \#1. This is equivalent to letting $\eta_{1}=0$, and defining $\eta_{s}$ as the relative delay between the signal from transmitter $s$ and the desired signal.

The receiver consists of three parts: the detector, the decoder and the feedback subsystem, as shown in Figure 1.4 (Page 8). We will introduce these parts in detail in the followings subsections.

In this section, we assume that the a priori probability distribution of all transmitted symbols is known to the receiver. However, this distribution can be arbitrary.

\footnotetext{
${ }^{1}$ Asynchronous transmission can be modeled by incorporating the transmission timing offset into $\eta_{s}$ and $\tau_{c, i}^{(s, a)}$.
} 


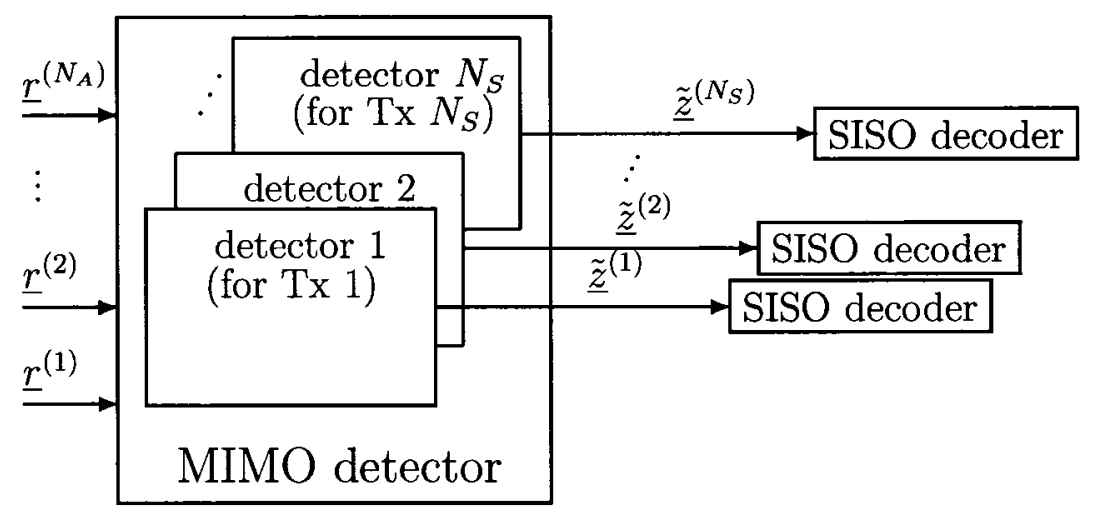

Figure 2.4: Block diagram of a MIMO detector.

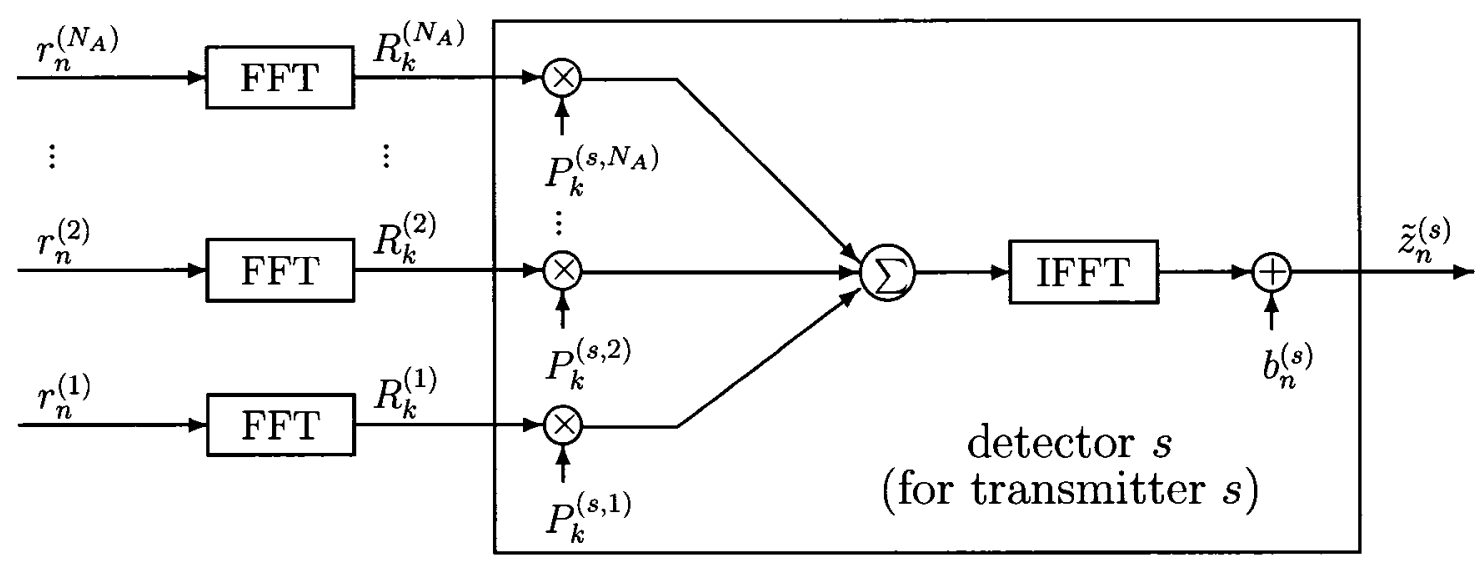

Figure 2.5: Block diagram of a detector.

We also assume that the receiver has perfect knowledge of $\eta_{s}, h_{l}^{(s, a)}$, and $N_{0}$, as would be provided by some hypothetical ideal channel estimation subsystems.

\subsubsection{Detector}

As Figure 2.4 shows us, a MIMO detector is composed of $N_{S}$ detectors, each of which is used to filter the ISI and the CCI for one of the $N_{S}$ transmitters. Detector $s$ takes the received frames, $\left[\underline{r}^{(1)}, \underline{r}^{(2)}, \ldots, \underline{r}^{\left(N_{A}\right)}\right]$, and generates $\underline{\tilde{z}}^{(s)}=\left[\begin{array}{llll}\tilde{z}_{0}^{(s)} & \tilde{z}_{1}^{(s)} & \ldots & \tilde{z}_{N_{T}-1}^{(s)}\end{array}\right]$, where $\tilde{z}_{n}^{(s)}$ is an estimate of $\tilde{v}_{n}^{(s)}=v_{n-\eta_{s}}^{(s)}$. 
To reduce complexity, the fast Fourier transform (FFT) and the inverse fast Fourier transform (IFFT) are used, and the filtering is done in the frequency domain. Figure 2.5 shows the block diagram of a single detector in a MIMO detector.

The filtering begins with the received frames. The FFT of each received symbol of the frame, $\underline{r}^{(a)}$, where $a \in\left\{1,2, \ldots, N_{A}\right\}$, is generated as

$$
R_{k}^{(a)}=\sum_{n=0}^{N_{T}-1} r_{n}^{(a)} e^{-j 2 \pi \frac{k n}{N_{T}}}
$$

where $k \in\left\{0,1,2, \ldots, N_{T}-1\right\}$.

All $R_{k}^{(a)}$ are then passed to each of the detectors for parallel filtering. In any detector, the input $R_{k}^{(a)}$ is first multiplied by the filter coefficient $P_{k}^{(s, a)}$. The summation of the products of the $N_{A}$ branches is then passed to IFFT. At last, an anti-biasing term $b_{n}^{(s)}$ is added to the IFFT output to form

$$
\tilde{z}_{n}^{(s)}=\frac{1}{N_{T}} \sum_{k=0}^{N_{T}-1} \sum_{a=1}^{N_{A}} P_{k}^{(s, a)} R_{k}^{(a)} e^{j 2 \pi \frac{k n}{N_{T}}}+b_{n}^{(s)},
$$

for $n \in\left\{0,1,2, \ldots, N_{T}-1\right\}$ and $s \in\left\{1,2, \ldots, N_{S}\right\}$.

As shown in Appendix A, when the minimum mean square error (MMSE) criterion is used to determine the filter coefficients, $P_{k}^{(s, a)}$, and the anti-biasing term, $b_{n}^{(s)}$, the detector output can be expressed in the form

$$
\tilde{z}_{n}^{(s)}=\frac{1}{N_{T}} \sum_{k=0}^{N_{T}-1} \sum_{a=1}^{N_{A}} P_{k}^{(s, a)}\left(R_{k}^{(a)}-\bar{R}_{k}^{(a)}\right) e^{j 2 \pi \frac{k n}{N_{T}}}+q_{0}^{(s)} \tilde{\bar{v}}_{n}^{(s)},
$$

where $\bar{R}_{k}^{(a)}=\mathcal{E}\left[R_{k}^{(a)}\right]$ is a locally generated estimate of the frequency-domain received samples, based on the a priori probability distribution of the transmitted symbols, as described in Subsection 2.3.3, $\tilde{\bar{v}}_{n}^{(s)}$ is a soft symbol, also described in Subsection 
2.3 .3 , and

$$
q_{0}^{(s)}=\frac{1}{N_{T}} \sum_{k=0}^{N_{T^{-}}-1} \sum_{a=1}^{N_{A}} P_{k}^{(s, a)} H_{k}^{(s, a)}
$$

where

$$
H_{k}^{(s, a)}=\sum_{l=0}^{L-1} h_{l}^{(s, a)} e^{-j 2 \pi \frac{k l}{N_{T}}}
$$

is the discrete Fourier transform (DFT) of the channel impulse response between transmitter $s$ and antenna $a$, which is assumed to be perfectly known at the receiver. The filter coefficient $P_{k}^{(s, a)}$ can be obtain by solving $N_{T}$ sets of $N_{A}$ linear equations as shown in Appendix A.

The detector works on frames of $N_{T}$ symbols, while the decoder operates on blocks of $N$ symbols. Therefore the detected frame, $\underline{\tilde{z}}^{(s)}$, is decomposed into blocks of $N$ samples, realigned with the corresponding transmitted symbol blocks. Referring back to Figure 2.3 , Block $m \in\left\{1,2, \ldots, N_{W}-1\right\}$ is given

$$
\underline{z}_{m}^{(s)}=\left[\begin{array}{llll}
\tilde{z}_{(m-1) N+\eta_{s}}^{(s)} & \tilde{z}_{(m-1) N+\eta_{s}+1}^{(s)} & \ldots & \tilde{z}_{m N+\eta_{s}-1}^{(s)}
\end{array}\right]
$$

and the last block is given by

$$
\underline{z}_{N_{W}}^{(s)}=\left[\begin{array}{llllll}
\tilde{z}_{\left(N_{W}-1\right) N+\eta_{s}}^{(s)} & \ldots & \tilde{z}_{N_{W} N-1}^{(s)} & 0 & \ldots & 0
\end{array}\right]
$$

where the zero-padding occurs because these detected samples are not part of the frame, so have not been detected yet, and are treated as erasures by the decoder. All $N_{W}$ blocks are passed to the decoder, even though decoding of Block $N_{W}$ will likely be unreliable, particularly if $\eta_{s}$ is large. 


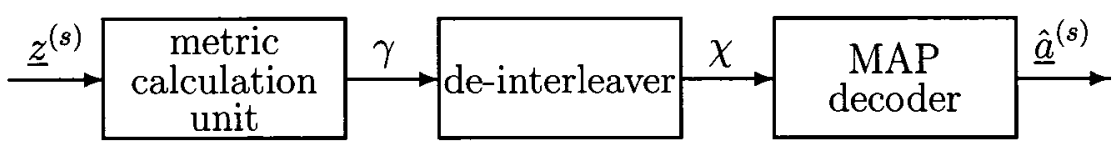

Figure 2.6: Block diagram of a decoder.

\subsubsection{Decoder}

The decoder uses the detected blocks, $\underline{z}_{m}^{(s)}$, which are noisy estimates of the transmitted symbol blocks, and attempts to determine the corresponding message words. Each of the $N_{W}$ blocks are decoded in turn, independently of each other. For notational convenience, we drop the block number index, $m$, so $\underline{z}^{(s)}$ is a detected block, corresponding to symbol block $\underline{v}^{(s)}$, which is based on message word $\underline{a}^{(s)}$.

The decoder consists of a metric calculation unit (MCU), a de-interleaver, and a MAP decoder, as shown in Figure 2.6. The MCU calculates the branch metrics for each transmitted bit, the de-interleaver reorders these metrics into the same order as the original code word, and the MAP decoder is used to decode the convolutional code.

\section{MCU}

The MCU calculates the branch metrics that will be used by the MAP decoder for the convolutional code. These metrics are based on the conditional likelihood function of sample $z_{n}^{(s)}$ being detected given that symbol $d_{n}^{(s)}=d$ was transmitted,

$$
\gamma_{n}^{(s)}(d)=f\left(z_{n}^{(s)} \mid d_{n}^{(s)}=d\right)
$$

for all $n \in\{0,1, \ldots, N-1\}$ and $d \in\{0,1, \ldots, M-1\}$. Thus for any $M$-ary constellation, $M$ metrics are calculated. 
Under the usual assumption that $z_{n}^{(s)}$ can be approximated as a Gaussian random variable, the likelihood function can be written as

$$
\gamma_{n}^{(s)}(d)=\frac{1}{\pi \bar{\sigma}_{z, s}^{2}} \exp \left\{-\frac{1}{\bar{\sigma}_{z, s}^{2}}\left|z_{n}^{(s)}-\mu_{n}^{(s)}(d)\right|^{2}\right\}
$$

where $\mu_{n}^{(s)}(d)$ and $\bar{\sigma}_{z, s}^{2}$ are the conditional mean and variance of $z_{n}^{(s)}$. From Appendix A, we know the conditional mean is

$$
\begin{aligned}
\mu_{n}^{(s)}(d) & =\mathcal{E}\left[z_{n}^{(s)} \mid d_{n}^{(s)}=d\right] \\
& =q_{0}^{(s)} \mathrm{SM}[d]
\end{aligned}
$$

where $q_{0}^{(s)}$ has been defined in Equation 2.11, and SM[·] denotes the constellation symbol mapping. The conditional variance is

$$
\begin{aligned}
\bar{\sigma}_{z, s}^{2} & =\frac{1}{N} \sum_{n=0}^{N-1} \mathcal{E}\left[\left|z_{n}^{(s)}-\mu_{n}^{(s)}(d)\right|^{2} \mid d_{n}^{(s)}=d\right] \\
& =q_{0}^{(s) *}\left(1-\bar{\sigma}_{v, s}^{2} q_{0}^{(s)}\right)
\end{aligned}
$$

where * denotes the complex conjugate and $\bar{\sigma}_{v, s}^{2}$ reflects the reliability of the feedback, as described in Subsection 2.3.3.

\section{De-interleaver}

Because the interleaver at the transmitter permutes the order of the code bits prior to transmission, the de-interleaver at the receiver is needed to restore the original ordering. Instead of reordering bits, though, the de-interleaver reorders the corresponding bit metrics, using the inverse of the permutation used by the transmitter. Prior to de-interleaving the bit metrics are determined from the symbol metrics of Equation 2.14 by marginalization $[16]$. 


\section{MAP decoder}

The MAP decoder is used to decode the convolutional code which is encoded at the transmitter. The MAP decoder selects the value with the maximum a posteriori probability as the estimate message bit $a_{n}^{(s)}=\arg \max _{a} \operatorname{Pr}\left\{a_{n}^{(s)}=a \mid \underline{z}^{(s)}\right\}$, where $a \in$ $\{0,1\}$. An algorithm for efficiently calculating the a posteriori probabilities was proposed by L.R. Bahl et al [18]. The algorithm actually produces two outputs: one is the a posteriori probabilities of the message bits, $\operatorname{Pr}\left\{a_{n}^{(s)}=a \mid \underline{z}^{(s)}\right\}$; the other is the a posteriori probabilities of the code bits, $\psi_{n}^{(s)}(c)=\operatorname{Pr}\left\{c_{n}^{(s)}=c \mid \underline{z}^{(s)}\right\}$. The latter is passed to the feedback subsystem and is used by the MIMO detector in the next iteration.

\subsubsection{Feedback Subsystem}

After decoding, the output of the SISO decoders gives us the estimated transmitted message words. The bit error rate performance is possibly quite poor due to the residual interference. However, we do have better knowledge of the transmitted data after decoding than we had before, so to achieve better decoding performance, we can feed-back the decoder results to the MIMO detector to better cancel the interference in the next iteration. As Figure 2.7 shows us, the feedback subsystem has $N_{S}$ feedback interleavers, $N_{S}$ soft symbol generators and an equivalent MIMO channel. 


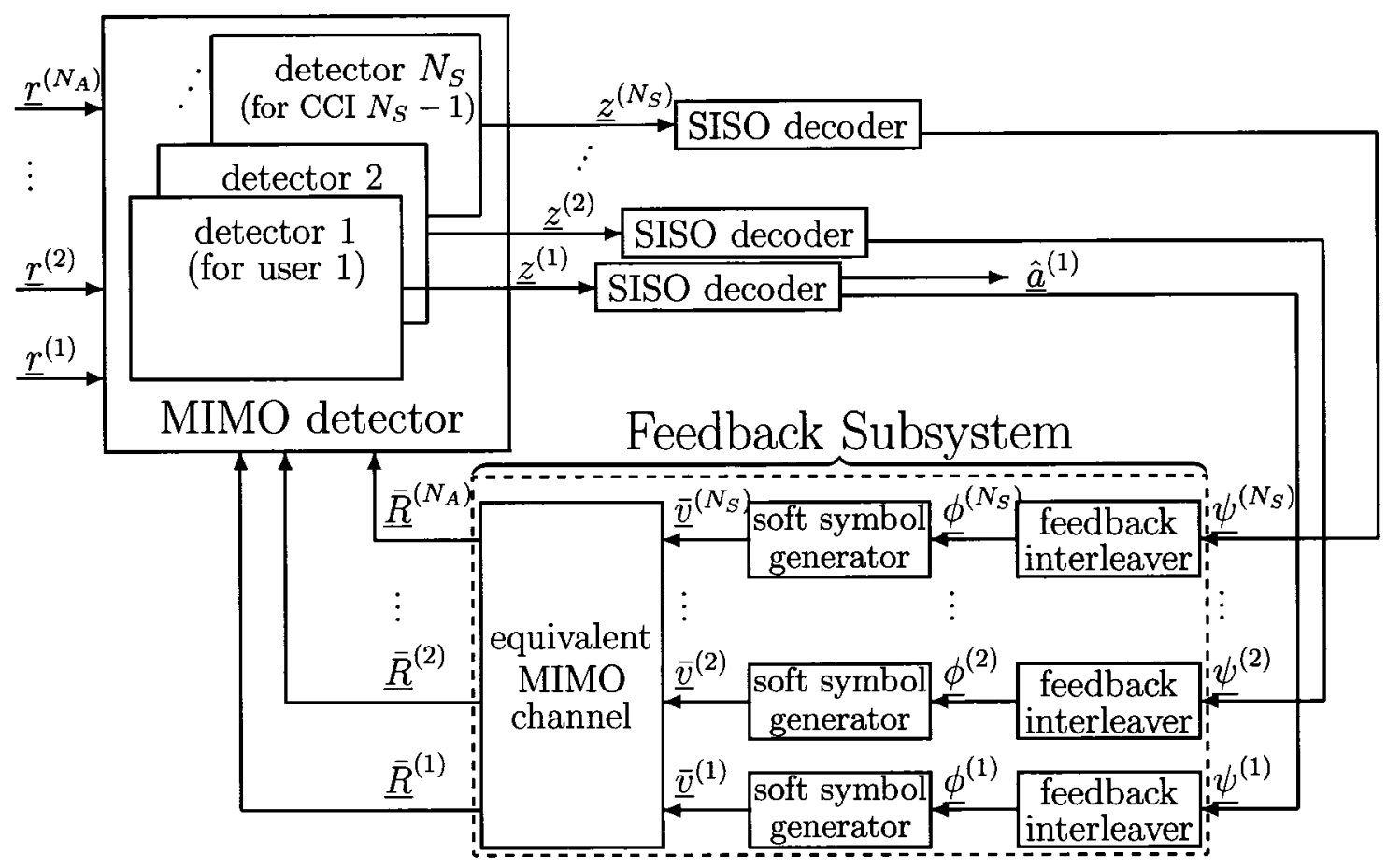

Figure 2.7: Block diagram of the feedback subsystem.

\section{Feedback Interleaver}

The feedback interleaver reorders the a posteriori code bit probabilities $\psi_{n}^{(s)}(c)$ produced by the MAP decoder using the same permutation as the interleaver at the transmitter. The interleaved bit probabilities are then combined into symbol probabilities, $\phi_{n}^{(s)}(d)$, which are used as the a priori probabilities, $\operatorname{Pr}\left\{d_{n}^{(s)}=d\right\}$, in the next iteration [16].

\section{Soft Symbol Generator}

The soft symbol generator is used to generate the expected transmitted symbols

$$
\bar{v}_{n}^{(s)}=\mathcal{E}\left[v_{n}^{(s)}\right]=\sum_{d=0}^{M-1} \operatorname{SM}[d] \operatorname{Pr}\left\{d_{n}^{(s)}=d\right\}=\sum_{d=0}^{M-1} \operatorname{SM}[d] \phi_{n}^{(s)}(d) .
$$


Visually, $\bar{v}_{n}^{(s)}$ represent a point on the constellation plane, although not necessarily one of the signal points. The location of the point depends on $\operatorname{Pr}\left\{d_{n}^{(s)}=d\right\}$. On one hand, if $\operatorname{Pr}\left\{d_{n}^{(s)}=d\right\}$ is large for some value of $d$, then $v_{n}^{(s)}$ will be close to $\operatorname{SM}[d]$. On the other hand, if $\operatorname{Pr}\left\{d_{n}^{(s)}=d\right\}$ is roughly equal for all $d$, then $v_{n}^{(s)}$ will be close to the origin.

The reliability of the soft symbols is given by their variance,

$$
\sigma_{v, n, s}^{2}=\mathcal{E}\left[\left|v_{n}^{(s)}-\bar{v}_{n}^{(s)}\right|^{2}\right]=\sum_{d=0}^{M-1}|\operatorname{SM}[d]|^{2} \operatorname{Pr}\left\{d_{n}^{(s)}=d\right\}-\left|\bar{v}_{n}^{(s)}\right|^{2}
$$

with smaller variance indicating higher reliability (that is, more certainty of its value). The average reliability used in Equation 2.16 is given by

$$
\bar{\sigma}_{v, s}^{2}=\frac{1}{N} \sum_{n=0}^{N-1} \sigma_{v, n, s}^{2}
$$

\section{Equivalent MIMO Channel}

The equivalent MIMO channel mimics the channel in the frequency domain. In particular, it simulates the MIMO channel model of Equation 2.6, except that the soft symbols, $\bar{v}_{n}^{(s)}$, are used in place of $v_{n}^{(s)}$, and the AWGN is ignored. That is, it calculates

$$
\bar{r}_{n}^{(a)}=\sum_{s=1}^{N_{S}} \sum_{l=0}^{L-1} h_{l}^{(s, a)} \bar{v}_{n-l-\eta_{s}}^{(s)}
$$

for all $a \in\left\{1,2, \ldots, N_{A}\right\}$ and $n \in\left\{0,1, \ldots, N_{T}-1\right\}$. As in Section 2.2, we use the subscript index of $\bar{v}_{n-l-\eta_{s}}^{(s)}$ to determine which block the soft symbol comes from, and the index into that block. Furthermore, we note that when $n-l-\eta_{s}$ is negative, the symbol comes from a block in a previously detect frame, not from one of the blocks just processed by the decoder. This is discussed more in Section 2.4. 
The FFT of the locally generated channel output estimates is then applied, giving

$$
\bar{R}_{k}^{(a)}=\sum_{n=0}^{N_{T}-1} \bar{r}_{n}^{(a)} \mathrm{e}^{-j 2 \pi \frac{k n}{N_{T}}},
$$

which are used in Equation 2.6. Alternatively, we can approximate the linear convolution of Equation 2.20 with a circular convolution, and calculate $\bar{R}_{k}^{(a)}$ directly as

$$
\bar{R}_{k}^{(a)}=\sum_{s=1}^{N_{S}} H_{k}^{(s, a)} \underline{\tilde{V}}^{(s)}
$$

where $\underline{\bar{V}}^{(s)}$ is the FFT of $\underline{\tilde{\bar{v}}}^{(s)}=\left[\begin{array}{llll}\tilde{\bar{v}}_{0}^{(s)} & \tilde{\bar{v}}_{1}^{(s)} & \ldots & \tilde{\bar{v}}_{N_{T}-1}^{(s)}\end{array}\right]$ with $\tilde{\bar{v}}_{n}^{(s)}=\bar{v}_{n-\eta_{s}}^{(s)}$.

\subsection{Frame Processing}

Each received frame is detected as a whole, then the detected frame for each user is divided into $N_{W}$ blocks, and each block is decoded independently. The decoded blocks are then recombined into frames by the equivalent MIMO channel, and the received frame is detected once again. This process is repeated for a few iterations, and then final estimates of the $N_{W}$ message words of the desired signal can be released to the data sink. The receiver then begins processing the next frame.

A problem with the approach is that only a portion of symbol block $N_{W}$ for each of the interferers is included in the frame. As a result, the decoders for these blocks is generally unable to reliably decode these blocks. Therefore, the detector, on the next iteration, is unable to effectively cancel the CCI in block $N_{W}$ of the desired signal. As a result, message word $N_{W}$ of the desired signal is subject to a much higher probability of error than the other message words. 
To overcome this problem, we suggest discarding the receiver output for message word $N_{W}$, and overlapping the frames by one block. That is, block $N_{W}-1$ of one frame becomes block 0 of the next frame, and block $N_{W}$ becomes block 1 . The receiver therefore produces $N_{W}-1$ received message words for each frame processed.

Finally, we note that the equivalent MIMO channel makes use of soft symbols from block 0 , which is not part of the frame. However, since block 0 of the current frame was block $N_{W}-1$ of the previous frame, we merely save the soft symbols of block $N_{W}-1$ on the final iteration of the previous frame, and employ them in the current frame.

\subsection{Novel Contributions of the System Model}

In general, the overall structure of our system model is very similar to J. Lin's model [16]. J. Lin's model is only able to handle synchronous received symbol blocks from all transmitters, processing on a block-by-block basis. To make our system asynchronous, we introduce the concept of the frame, which consists of $N_{W}$ blocks, to overcome the symbol offset among different transmitters. Therefore, some steps are needed to fulfill the block-to-frame and the frame-to-block transformation.

For the MIMO detector, we introduce not only the concept of the frame to overcome the symbol offset (coarse offset), but also the random ISI-tap shift within a sampling period (fine offset), to model a real asynchronous system. The output of the MIMO detector is decomposed into blocks for further processing. For the SISO decoders, the whole structure comes from J. Lin's model, except that the estimated 
message word output of interferers is omitted. For the feedback subsystem, our model is also similar to J. Lin's. The only difference is that a block-to-frame re-construction is needed in the equivalent MIMO channel.

In addition, in J. Lin's model cyclic prefixes are inserted before each block prior to transmission, so the linear convolution of the transmitted signal with the channel impulse response becomes equivalent to a circular convolution. With our system the cyclic prefixes (and their associated bandwidth overhead) are not used. On one hand, because the system is asynchronous, the convolution is never circular for all users, even with the circular prefix. On the other hand, even though the detector assumes the channel convolution is circular, because processing is done over frames instead of blocks, the "edge effect" distortion due to this approximation is lessened. 


\section{Chapter 3}

\section{System Performance}

In this chapter, we evaluate the system performance of the proposed asynchronous system model using computer simulation.

To set a benchmark for comparisons, we consider a system with only one transmitter. In such a system, there is no CCI but the ISI and noise still exist. For figures in this chapter, we denote such a system as "without CCI". Apparently, the performance of such a system is the best we can achieve under the specific ISI channel model. If our system is effective at canceling CCI, it should be able to deliver performance that is close to the performance of the "without CCI" system.

When IC is used to reduce the CCI, we denote it as "IC". To show the improvement that the interference canceler (IC) offers, we also draw the curves for the case when no IC used. The legend "no IC" denotes that no interference canceling is used at the receiver, while the legend "no IC equivalent noise" means that the interference is modeled and simulated as AWGN of equivalent power, instead of an actual databearing signal. 


\begin{tabular}{|l|r|}
\hline Parameter & Value \\
\hline \hline Blocks per truncated frame $\left(N_{W}\right)$ & 4 \\
\hline Symbols per block $(N)$ & 1024 \\
\hline Convolutional code & $(7,5)_{8}$ \\
\hline Constellation & QPSK \\
\hline Number of iterations & 5 \\
\hline Rolloff factor $(\beta)$ & 0.2 \\
\hline
\end{tabular}

Table 3.1: Shared simulation parameters.

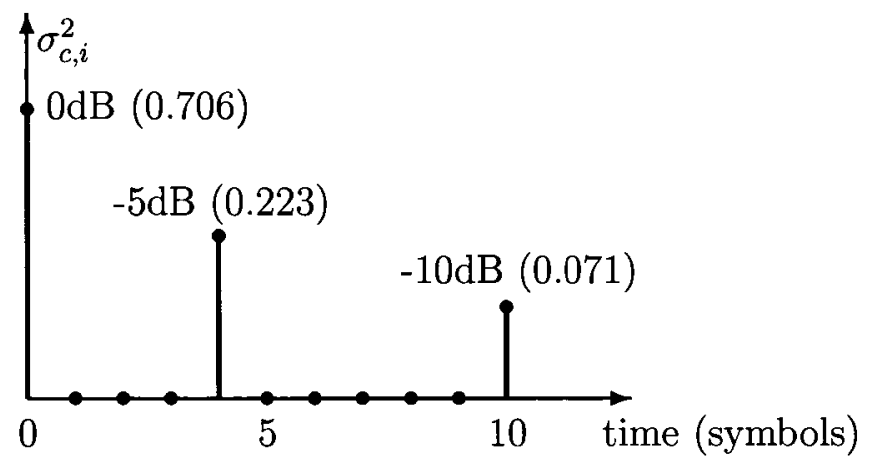

Figure 3.1: SUI-5 power delay profile.

In this chapter, we mostly use BER vs. SNR and WER vs. SNR graphs for comparison. WER (word error rate) is directly related to the throughput, which is used for later comparison.

Unless otherwise stated, the shared parameters for all simulation are shown in Table 3.1.

\subsection{System Performance for SUI-5 Channel}

The power delay profile of the SUI-5 channel (with a symbol rate of 1 MBaud) is shown in Figure 3.1. The three taps span eleven symbols. 


\subsubsection{Performance for $N_{S}=2, N_{A}=1$}

In this subsection, we focus on the channel consisting of two transmitters and one receive antenna $\left(N_{S}=2, N_{A}=1\right)$.

In our model, the CCI power at the receiver is measured relative to the signal power. We use $\Delta$ to denote the discrepancy. Therefore, $\Delta(\mathrm{dB})=P_{S}(\mathrm{~dB})-P_{I}(\mathrm{~dB})$, where $P_{S}$ and $P_{I}$ represent the signal power and the CCI power at the receiver, respectively. The following parts of this subsections will present the system performance for various $\Delta$.

\section{Performance for $\Delta=0 \mathrm{~dB}$}

Figure 3.2 shows us the BER and WER performance for three different modes of synchronization on the SUI-5 channel with $N_{S}=2, N_{A}=1$, and $\Delta=0 \mathrm{~dB}$. The legend "fully synchronous" denotes the performance of a fully synchronous system, where $\eta_{s}=0$ for all $s$ and $\tau_{c, i}^{(s)}=\bar{\tau}_{c, i}$ for all $i$; the legend "partially synchronous" represents a symbol-synchronous system, where $\eta_{s}$ is randomly selected (with equal probabilities) from $\{0,1, \ldots, N-1\}$ for $s>1$ and $\eta_{1}=0$, and $\tau_{c, i}^{(s)}=\bar{\tau}_{c, i}$; the legend "fully asynchronous" means a fully asynchronous system, where $\eta_{s}$ is randomly selected as for the partially synchronous case, and $\tau_{c, i}^{(s)}$ is randomly selected (with uniform distribution) from $\left[\bar{\tau}_{c, i}-T / 2, \bar{\tau}_{c, i}+T / 2\right)$. In this figure, the "fully synchronous" curve and the "partially synchronous" curve can barely be distinguished, which means that we suffer no penalty from block-synchronization to symbol-synchronization. The penalty for the fully-asynchronous case is less than $0.5 \mathrm{~dB}$. The following performance 


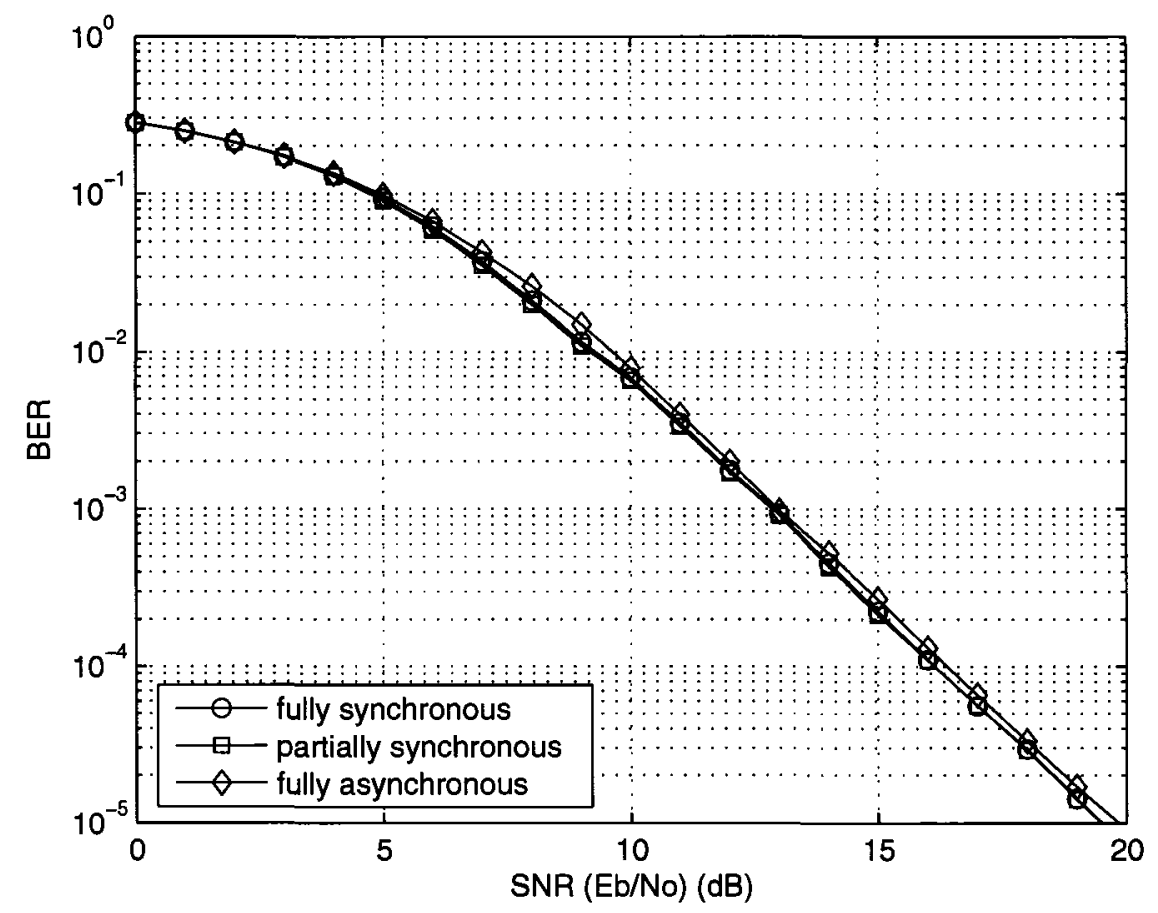

(a) BER performance

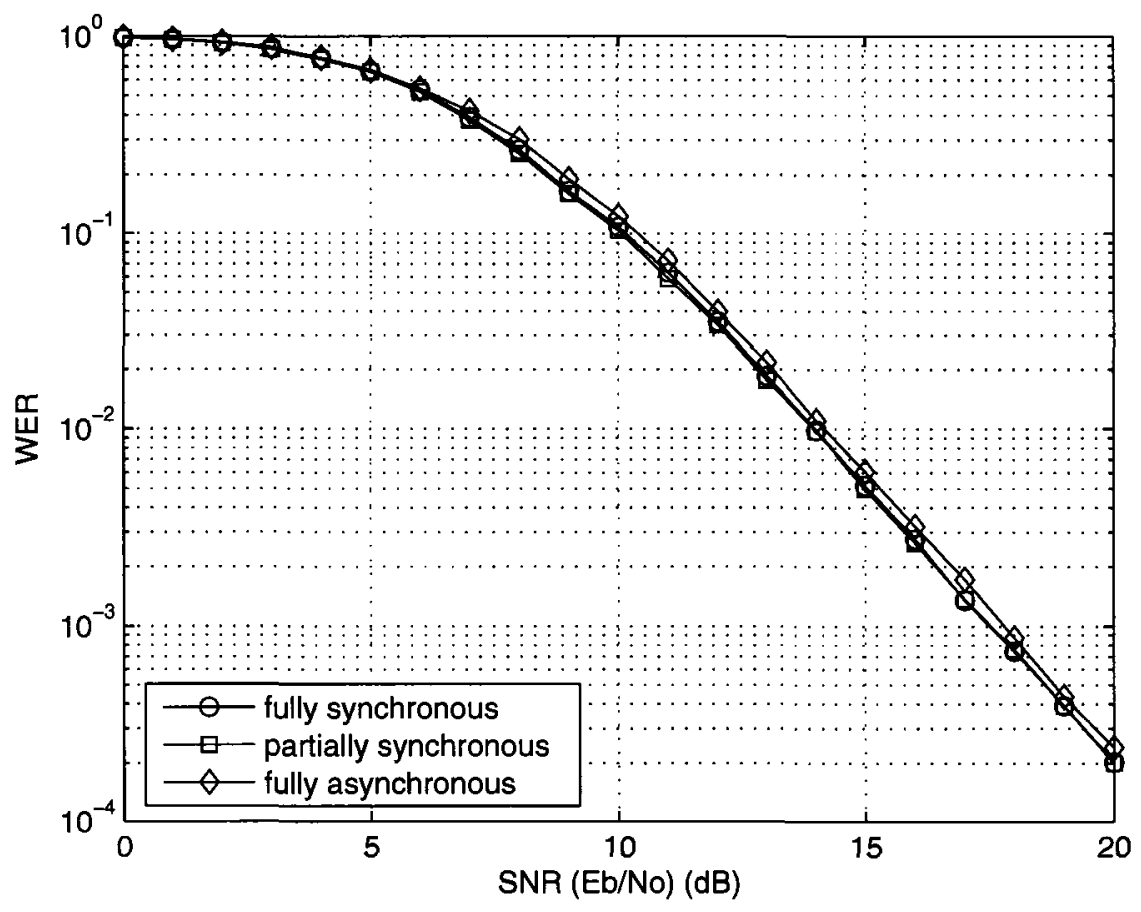

(b) WER performance

Figure 3.2: Error performance for different modes of synchronization on the SUI-5 channel with $N_{S}=2, N_{A}=1, \Delta=0 \mathrm{~dB}$. 


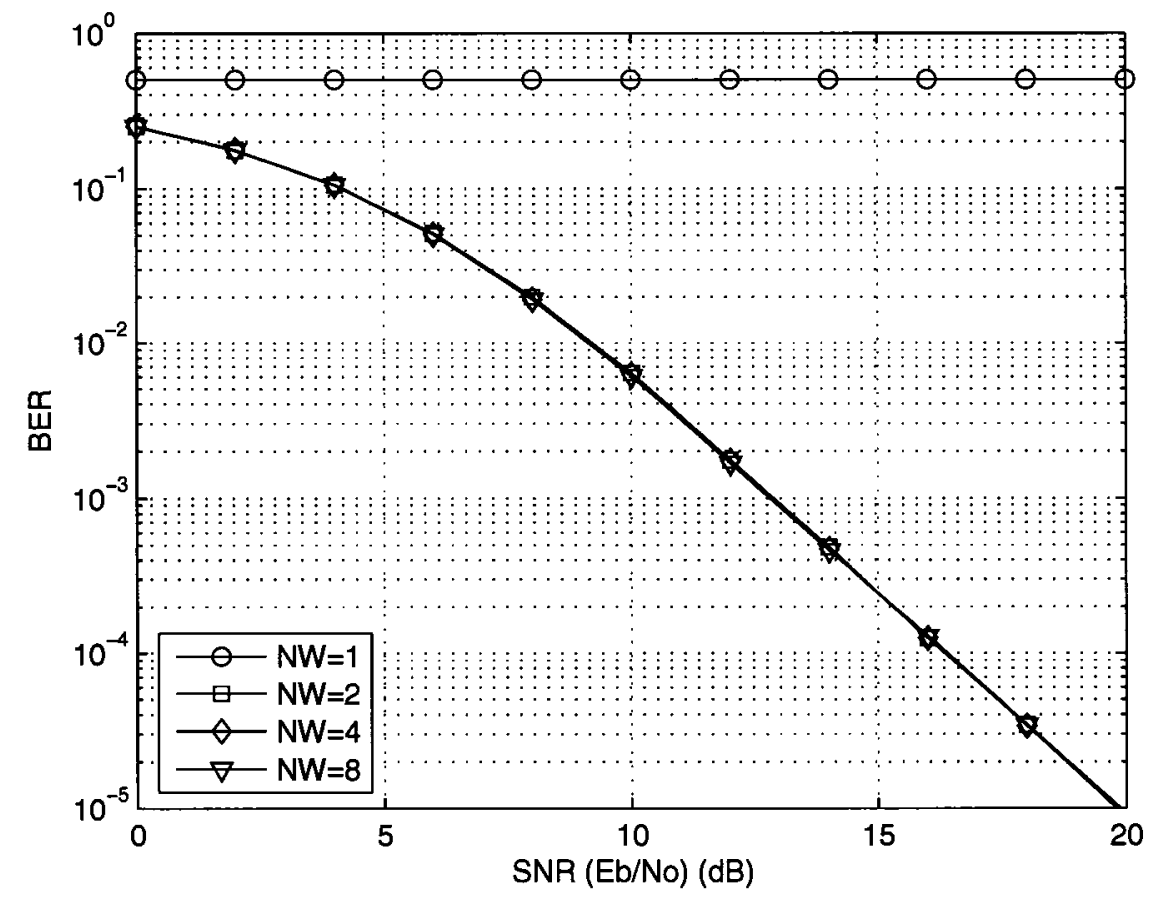

Figure 3.3: Block 1 BER performance of different frame structures on SUI-5 channel with $N_{S}=2, N_{A}=1, \Delta=0 \mathrm{~dB}$.

discussion is all focused on the fully-asynchronous case.

Figure 3.3 shows the BER performance of block 1 in the different frame structures (Figure 2.3, Page 18). When $N_{W}=1$, the model does not work, which means that the block-based structure can not process asynchronous data. The other three curves are almost superimposed, suggesting that the option of $N_{W}$ has little effect on BER performance. Recalling that the decoding of block 1 benefits from the assumption of perfect decoding of block 0 , we use block 2 of $N_{W}=4$ frame structure for the following performance discussion.

Figure 3.4 shows us the BER and WER performance, when $\Delta=0 \mathrm{~dB}$. We can see that when no attempt is made to cancel the CCI, the performance is very poor, 


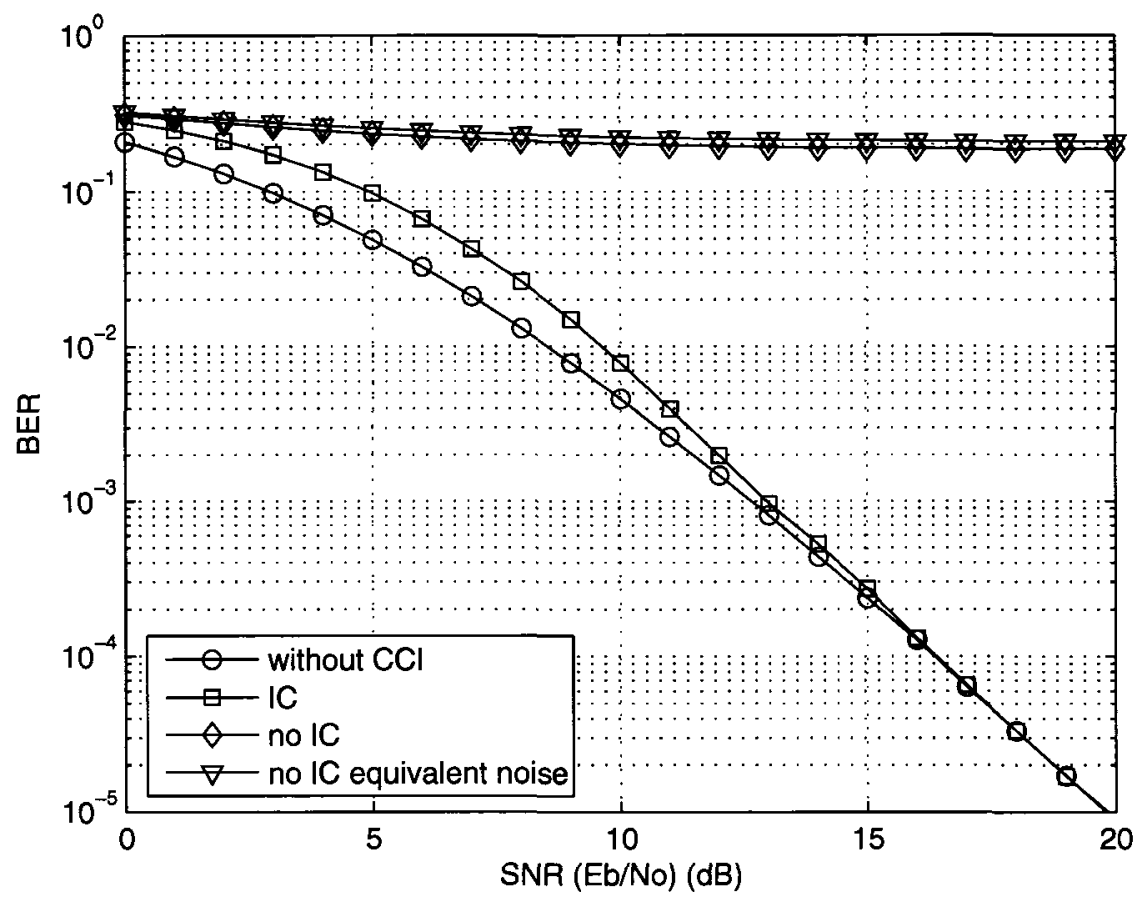

(a) BER performance

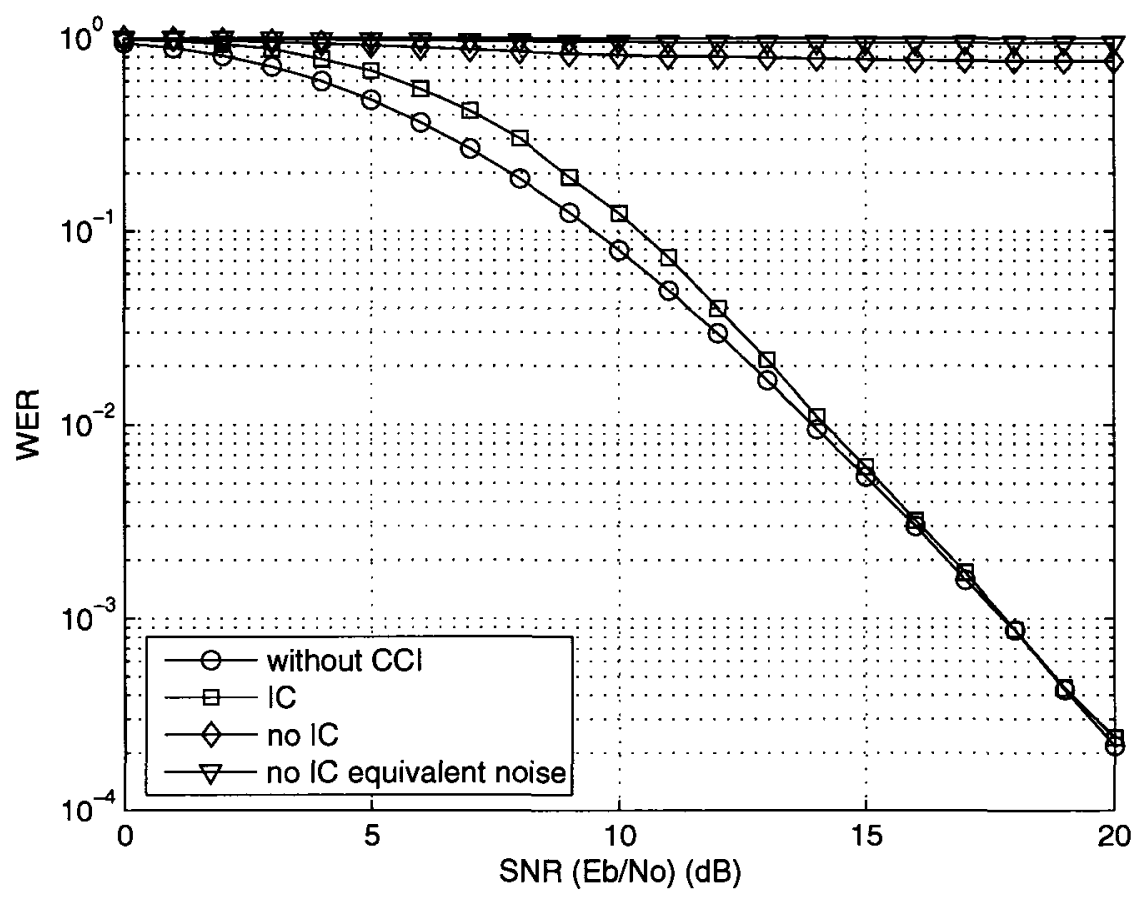

(b) WER performance

Figure 3.4: Performance on SUI-5 channel with $N_{S}=2, N_{A}=1, \Delta=0 \mathrm{~dB}$. 
with a very high error floor. This is because when $\Delta=0 \mathrm{~dB}$ the interferer is as strong as the desired signal. Since the signal to interferer-plus-noise ratio (SINR) is always less than $0 \mathrm{~dB}$ (regardless of how high the SNR is), it is impossible to reliably detect the desired signal. This is true regardless of whether the interferer is an actual data-bearing signal ("no IC") or is modeled as AWGN with the same power ("no IC equivalent noise"). Although the performance with the data-bearing interferer is slightly better, the equivalent noise model is sufficiently accurate.

Even though the system performance is very poor when the CCI is ignored, the proposed IC works very well, as indicated by the "IC" curve. We see that the error floor does not occur, so reliable communication becomes possible even in the presence of such a strong interferer. The effectiveness of the proposed receiver is truly evident when we compare the performance of the IC with the "without CCI" case. At high SNR the two curves converge, indicating that the IC is able to completely eliminate the CCI. This is quite remarkable for a receiver with only one antenna $\left(N_{A}=1\right)$.

We also note that at low SNR there is a slight gap between the performance of the IC and the "without CCI" case. This is due to residual interference that cannot be canceled. More precisely, at low SNR there is a higher probability that the decoder will be unable to correctly decode the CCI code word. When this happens, the CCI cannot be fully removed, so the decoder for the desired signal is less likely to be able to decode its code word, leading to a higher probability of error than if the CCI was not present. 


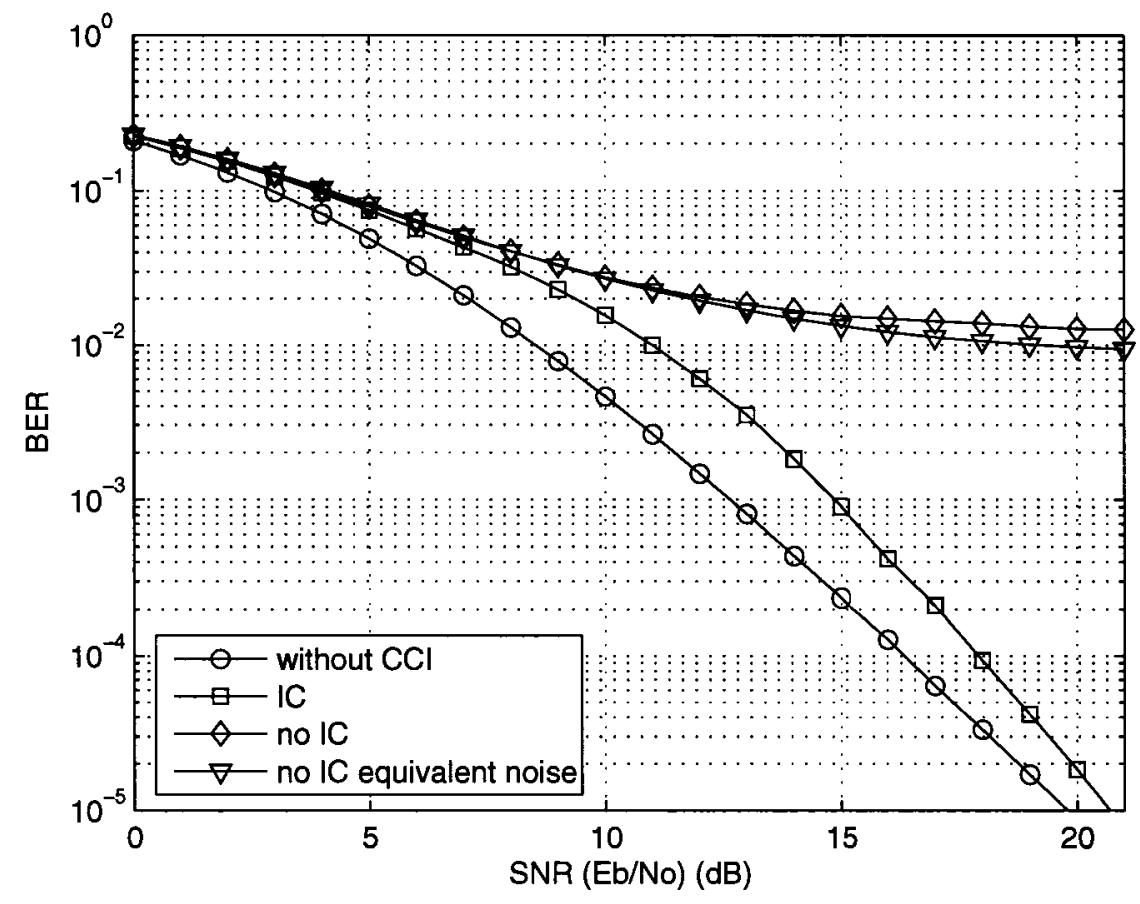

(a) BER performance

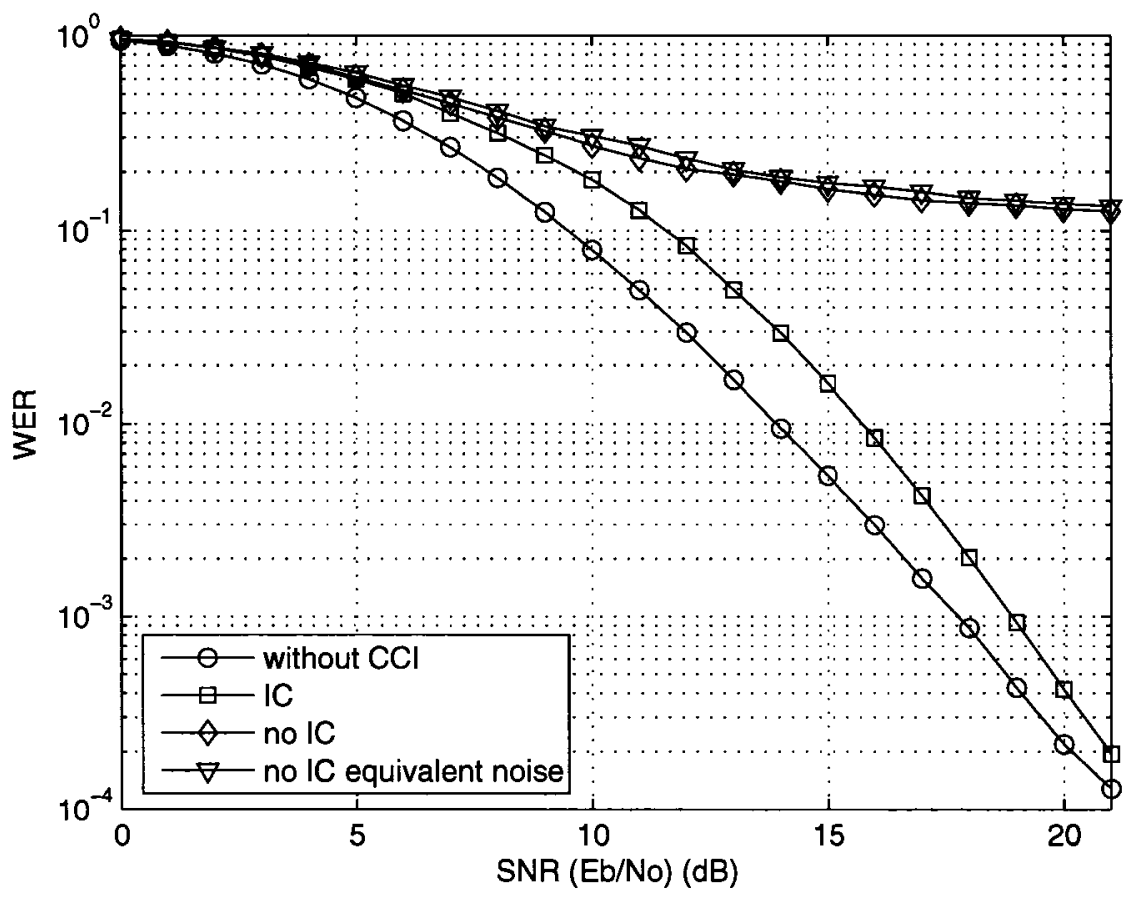

(b) WER performance

Figure 3.5: Performance on SUI-5 channel with $N_{S}=2, N_{A}=1, \Delta=9 \mathrm{~dB}$. 
Performance for $\Delta=9 \mathrm{~dB}$

Figure 3.5 shows the BER and WER performance for $\Delta=9 \mathrm{~dB}$. We again observe an error floor in the "no IC" cases, although at a lower level than for $\Delta=0 \mathrm{~dB}$. This is because the SINR approaches $9 \mathrm{~dB}$ as the SNR increases, and the weaker interferer is less of an impediment to reliable communication.

We also note that the IC is able to significantly improve the performance, although the CCI is not eliminated as thoroughly as in the $\Delta=0 \mathrm{~dB}$ case. In fact, we observe a slight bump in the "IC" curve compared to the "without CCI" case. At low SNR, and at high SNR, the "IC" curve is close to the "without CCI" curve, but at moderate SNR the discrepancy is more significant, with a maximum difference at about $12 \mathrm{~dB}$. This bump is due to the fact that, at low SNR, although we are unable to cancel the $\mathrm{CCI}$, the BER (and WER) is governed by the noise, so the relatively weak CCI does not degrade the performance much. As the SNR increases, the noise becomes less of a problem, so the BER decreases, but the CCI, which is now about as strong as the noise, still can not be effectively canceled, limiting any improvement in the BER. This is because at SNR $=12 \mathrm{~dB}$ and $\Delta=9 \mathrm{~dB}$, for example, the interference-to-noise ratio is only $3 \mathrm{~dB}$, so the $\mathrm{IC}$ is unable to detect (and therefore cancel) the CCI. As the SNR increases further, the interference-to-noise ratio also increases, and the IC becomes much more effective at detecting and canceling the CCI. 


\section{Performance for other $\Delta$}

In order to further verify the conclusion of the bump, we present the BER for other values of $\Delta$, as shown in Figures 3.6, 3.7, 3.8, and 3.9. From these figures, we can clearly see that the locations of bumps are around $\mathrm{SNR}=\Delta+3 \mathrm{~dB}$. We also observe that at low SNR, a strong interferer causes more degradation to system performance than a weak one, whereas at high SNR, moderately weak interferers are more problematic.

\subsubsection{Performance for $N_{S}=2, N_{A}=2$}

In this subsection, we increase the number of antennas from 1 to $2\left(N_{S}=2, N_{A}=2\right)$. Since the extra antenna increases the diversity, we may expect better performance than the $N_{S}=2, N_{A}=1$ case.

As we expected, when $\Delta=0 \mathrm{~dB}$, Figure 3.10 shows us the significant performance improvement compared to the $N_{S}=2, N_{A}=1$ counterpart. To achieve a BER of $10^{-5}$, the $N_{S}=2, N_{A}=1$ case needs the SNR as high as $20 \mathrm{~dB}$, while the $N_{S}=2, N_{A}=2$ case only needs the SNR to be $10 \mathrm{~dB}$.

Figure 3.11 shows the corresponding results for $\Delta=3 \mathrm{~dB}$. We notice that the extra antenna reduces the bump, which means that the receiver is even more effective at canceling the interference. This is another benefit of antenna diversity. 


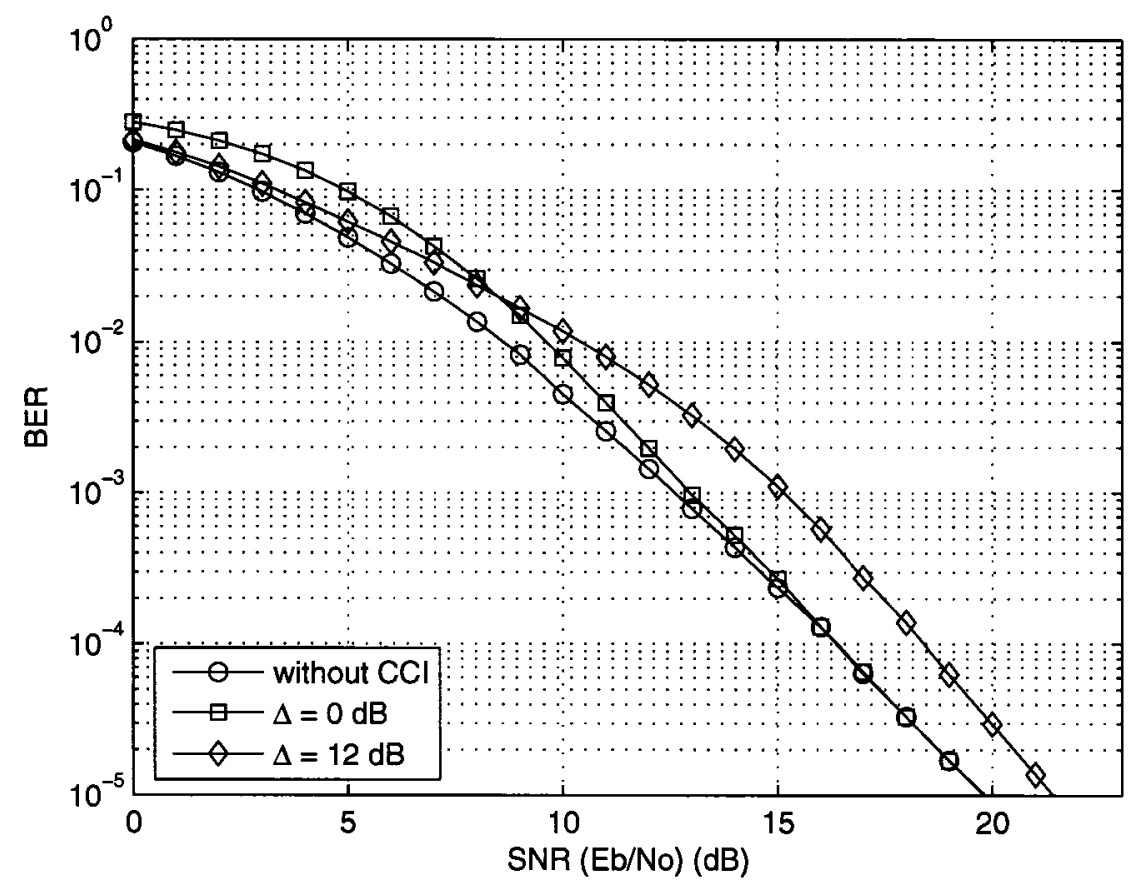

Figure 3.6: BER performance on SUI-5 channel with $N_{S}=2, N_{A}=1$.

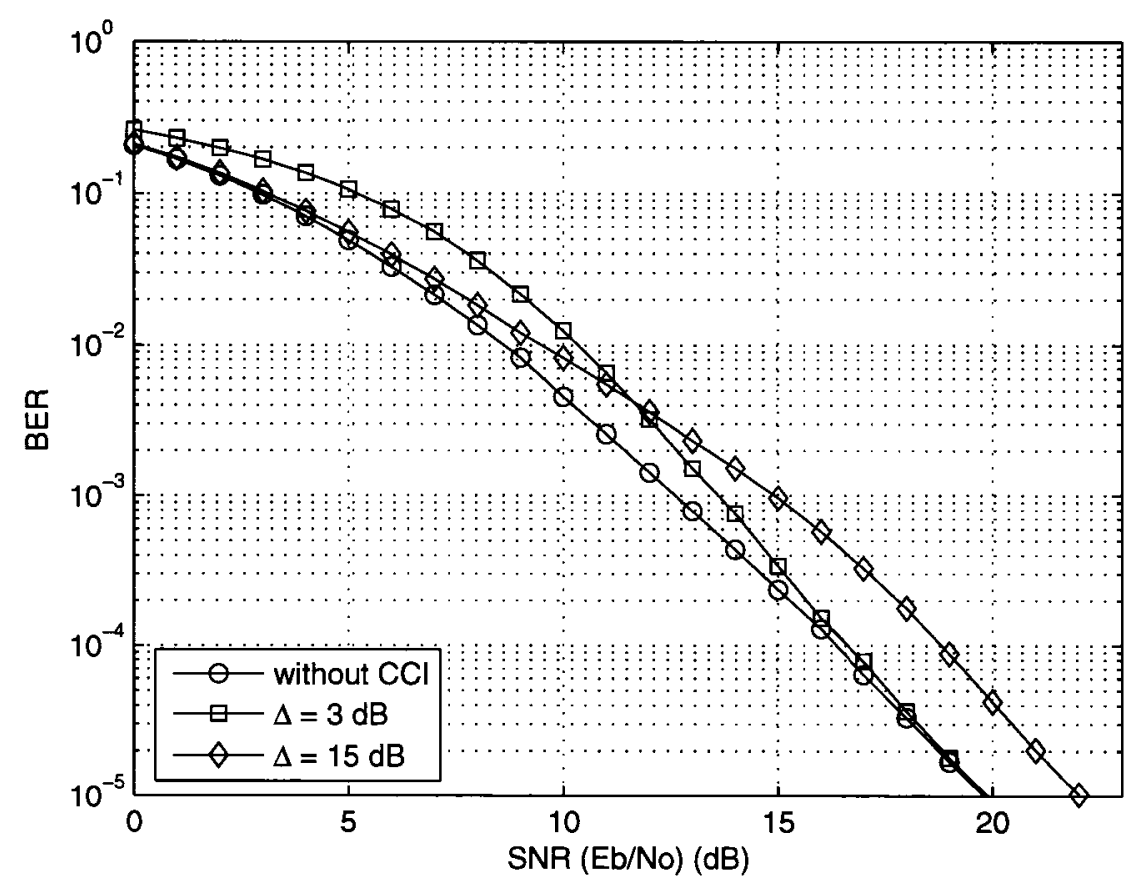

Figure 3.7: BER performance on SUI-5 channel with $N_{S}=2, N_{A}=1$. 


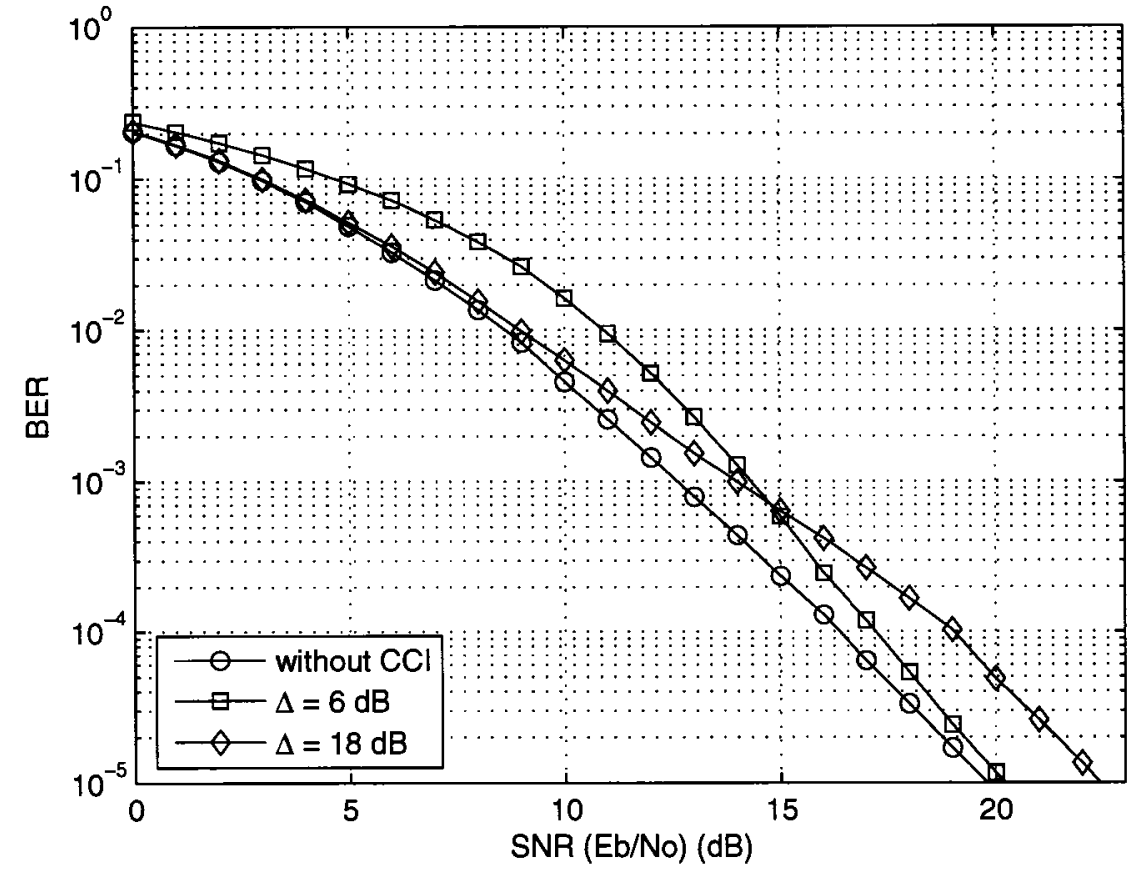

Figure 3.8: BER performance on SUI-5 channel with $N_{S}=2, N_{A}=1$.

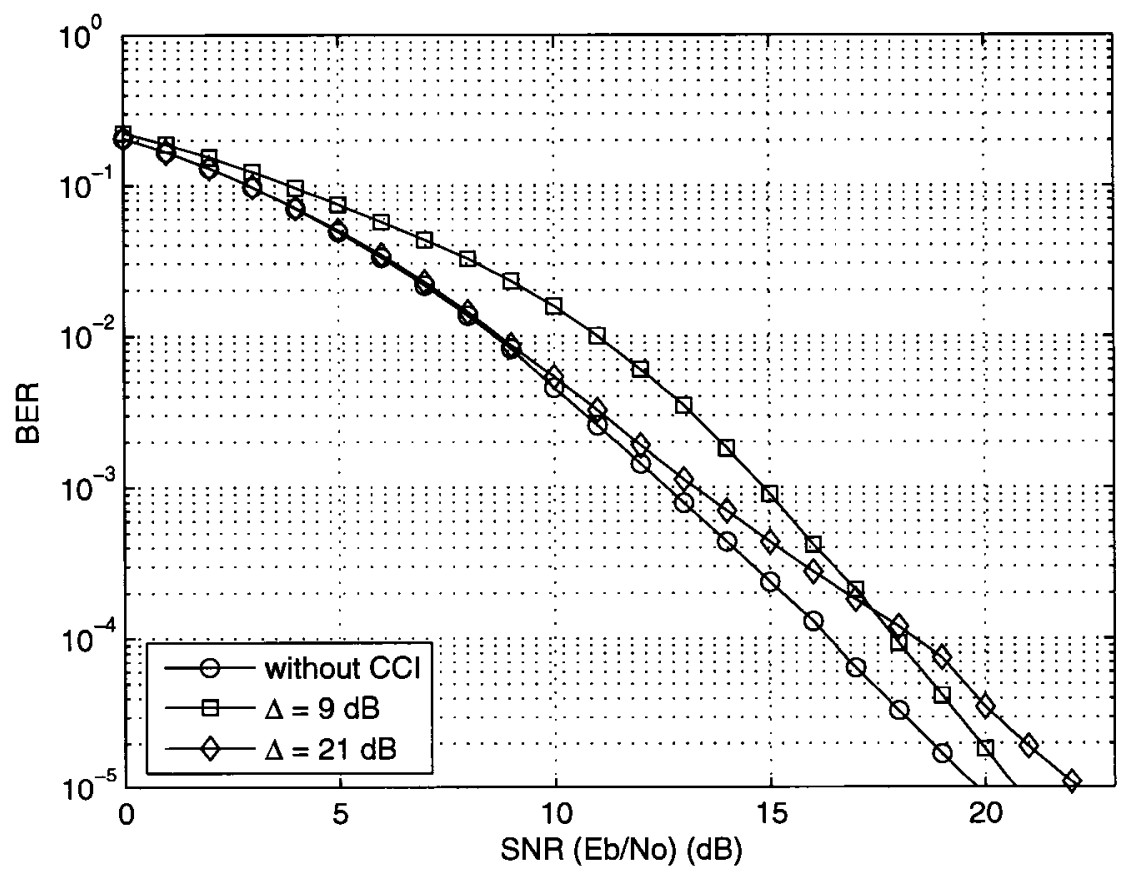

Figure 3.9: BER performance on SUI-5 channel with $N_{S}=2, N_{A}=1$. 


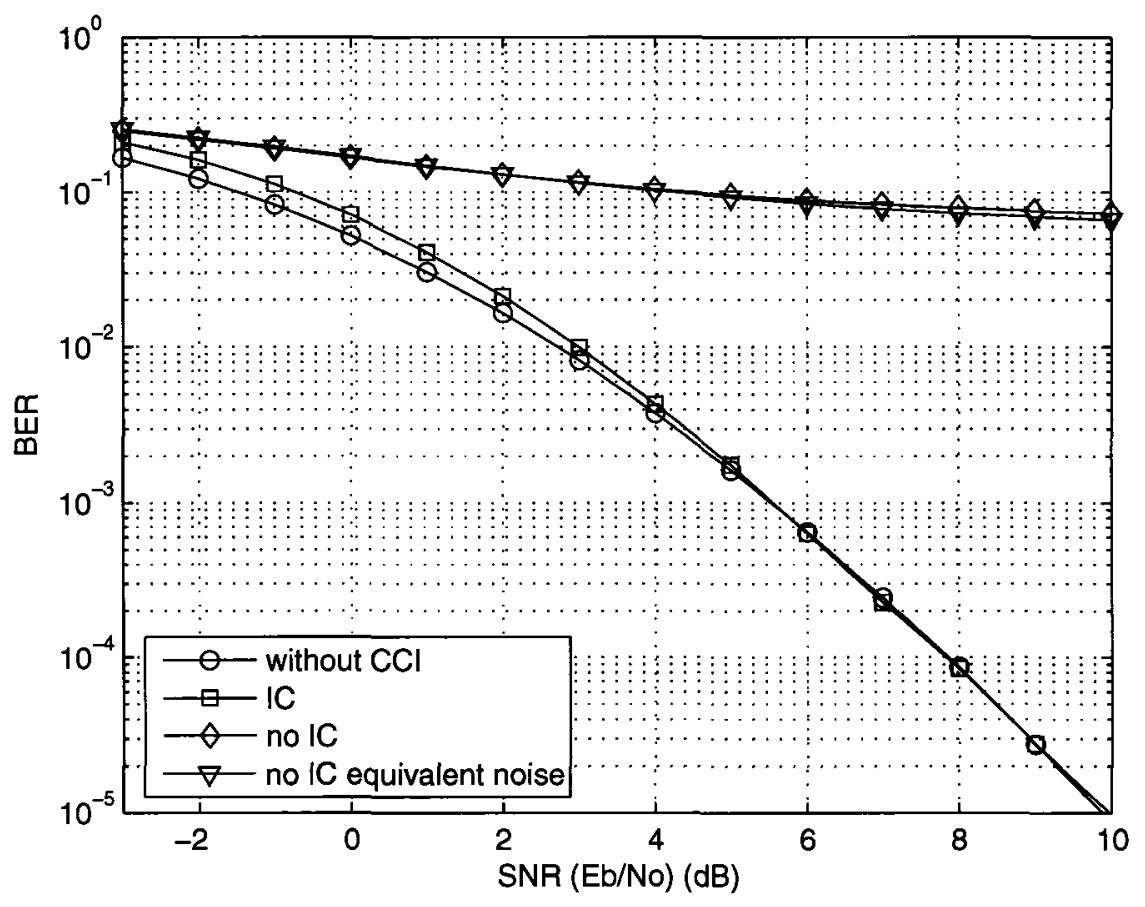

(a) BER performance

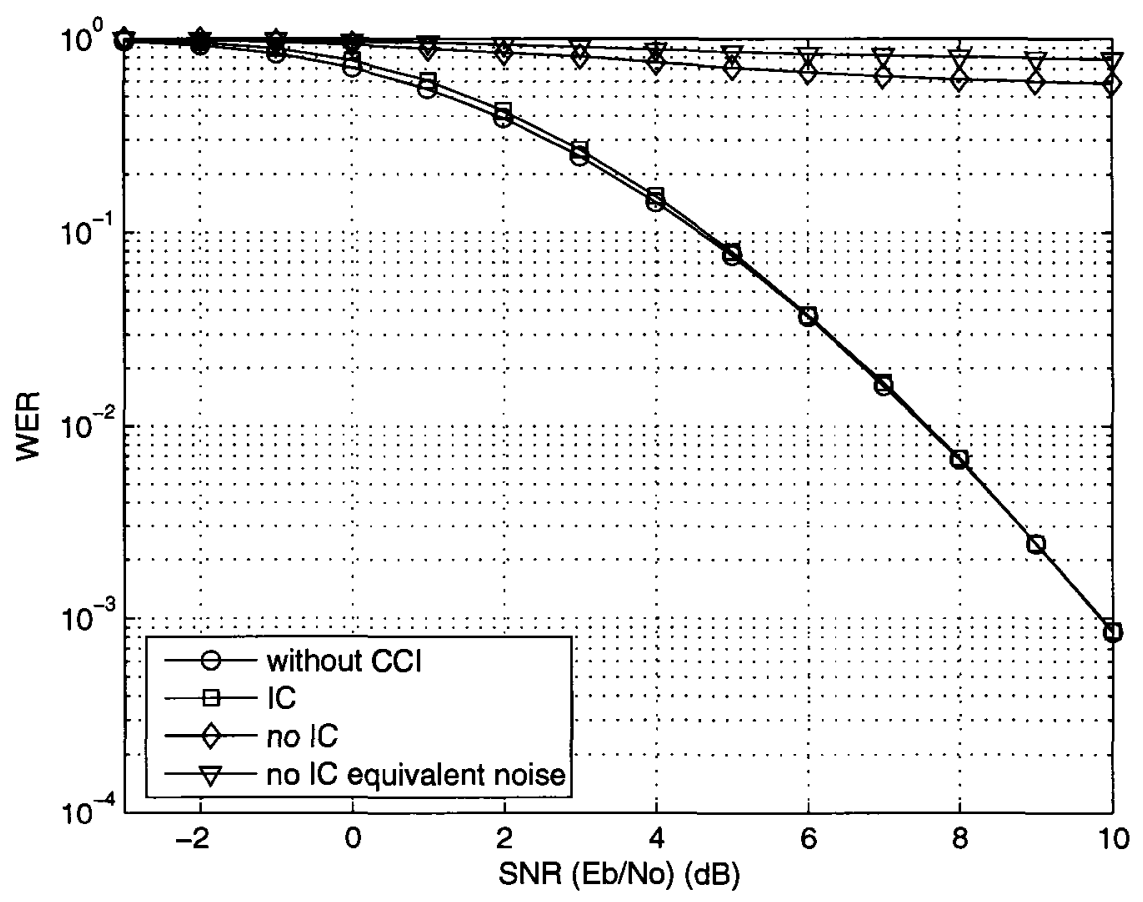

(b) WER performance

Figure 3.10: Performance on SUI-5 channel with $N_{S}=2, N_{A}=2, \Delta=0 \mathrm{~dB}$. 


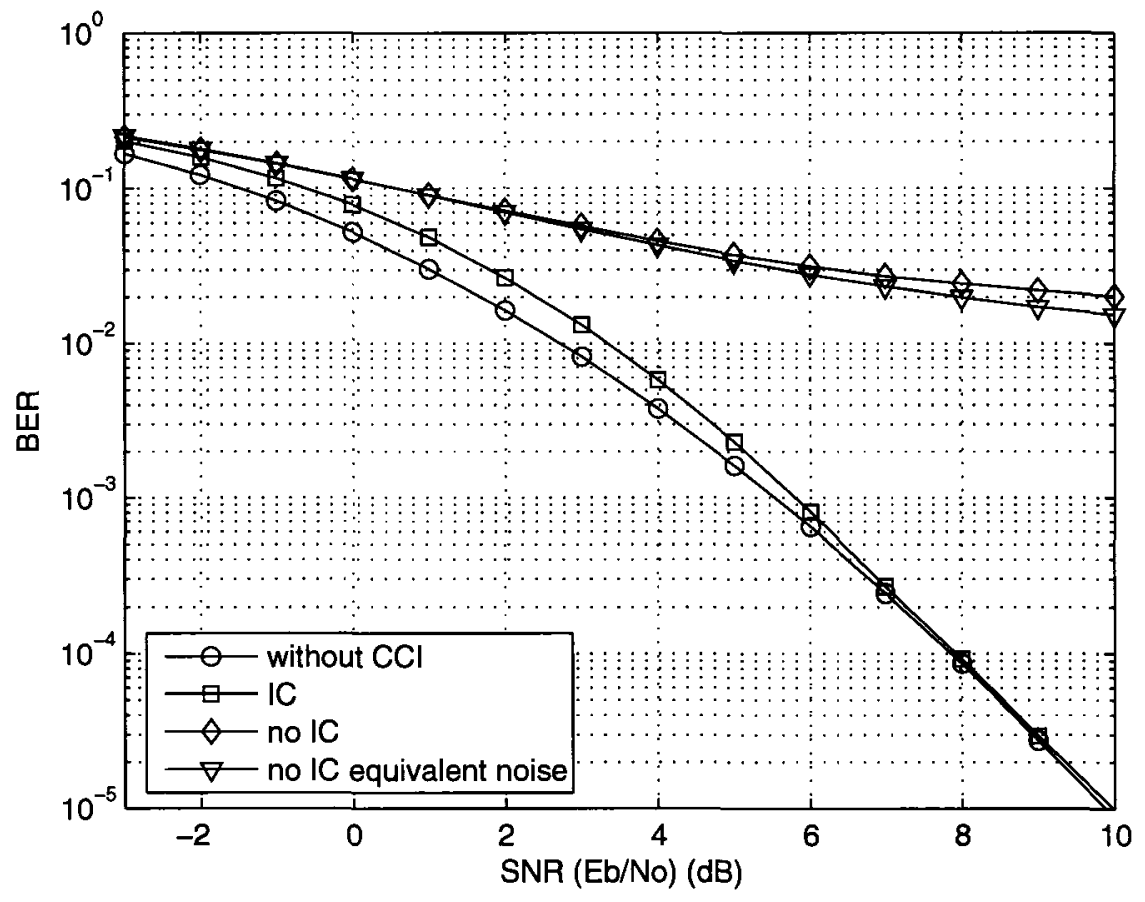

(a) BER performance

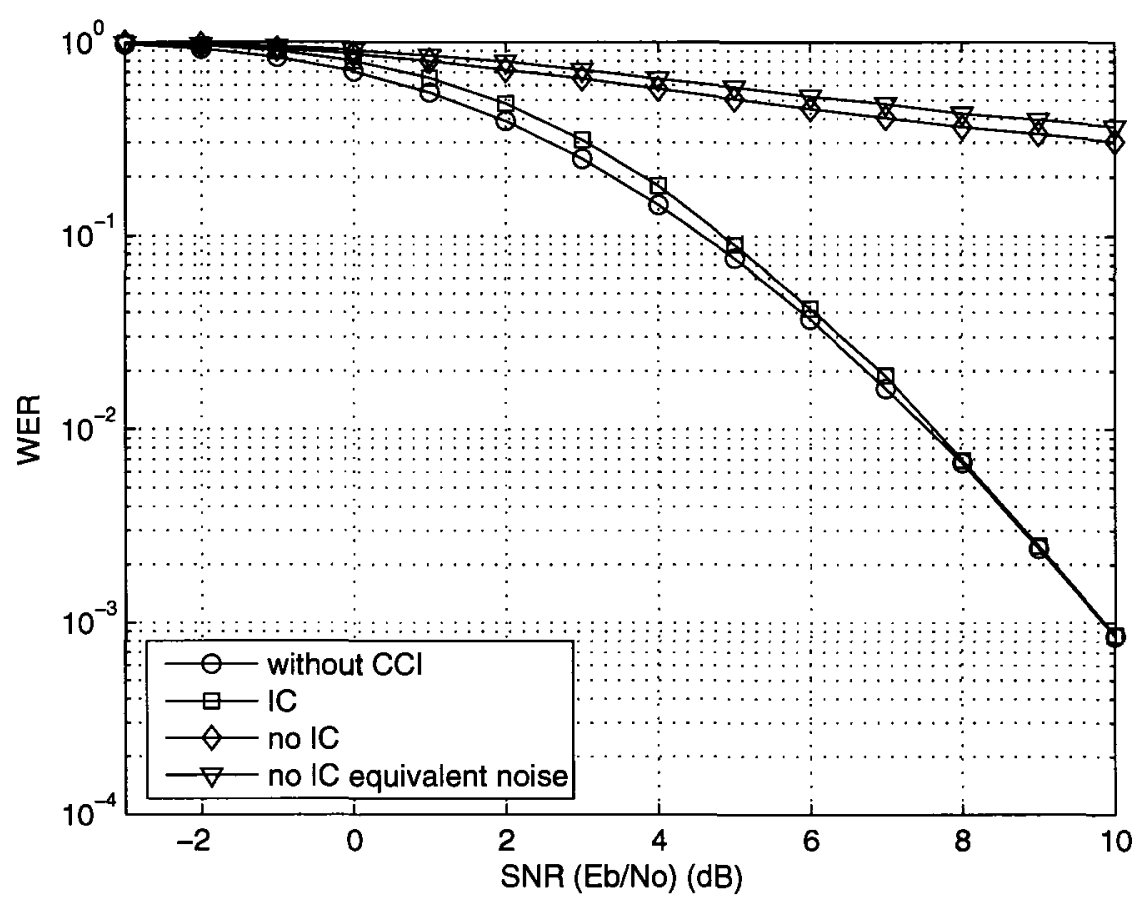

(b) WER performance

Figure 3.11: Performance on SUI-5 channel with $N_{S}=2, N_{A}=2, \Delta=3 \mathrm{~dB}$. 


\subsubsection{Performance for $N_{S}=3, N_{A}=2$}

Since a two-antenna receiver can better cancel the interference, we now increase the number of transmitters from 2 to $3\left(N_{S}=3, N_{A}=2\right)$, so there are now two interferers.

Figure 3.12 shows the BER and WER performance of this case when $\Delta=0$ dB. If we compare Figure 3.12 with Figure 3.10 , we may find that two figures are similar. However, the bump of the IC curve in Figure 3.12 is noticeably bigger. The reason is obvious: the additional interferer increases the overall probability of incorrect decoding.

Figure 3.13 shows the performance for $N_{S}=3, N_{A}=2$ for $\Delta=3 \mathrm{~dB}$. We can still clearly see the bump, but it occurs at a lower SNR, at SNR $=\Delta$ instead of $\mathrm{SNR}=\Delta+3 \mathrm{~dB}$ as in the $N_{S}=2, N_{A}=1$ case. 


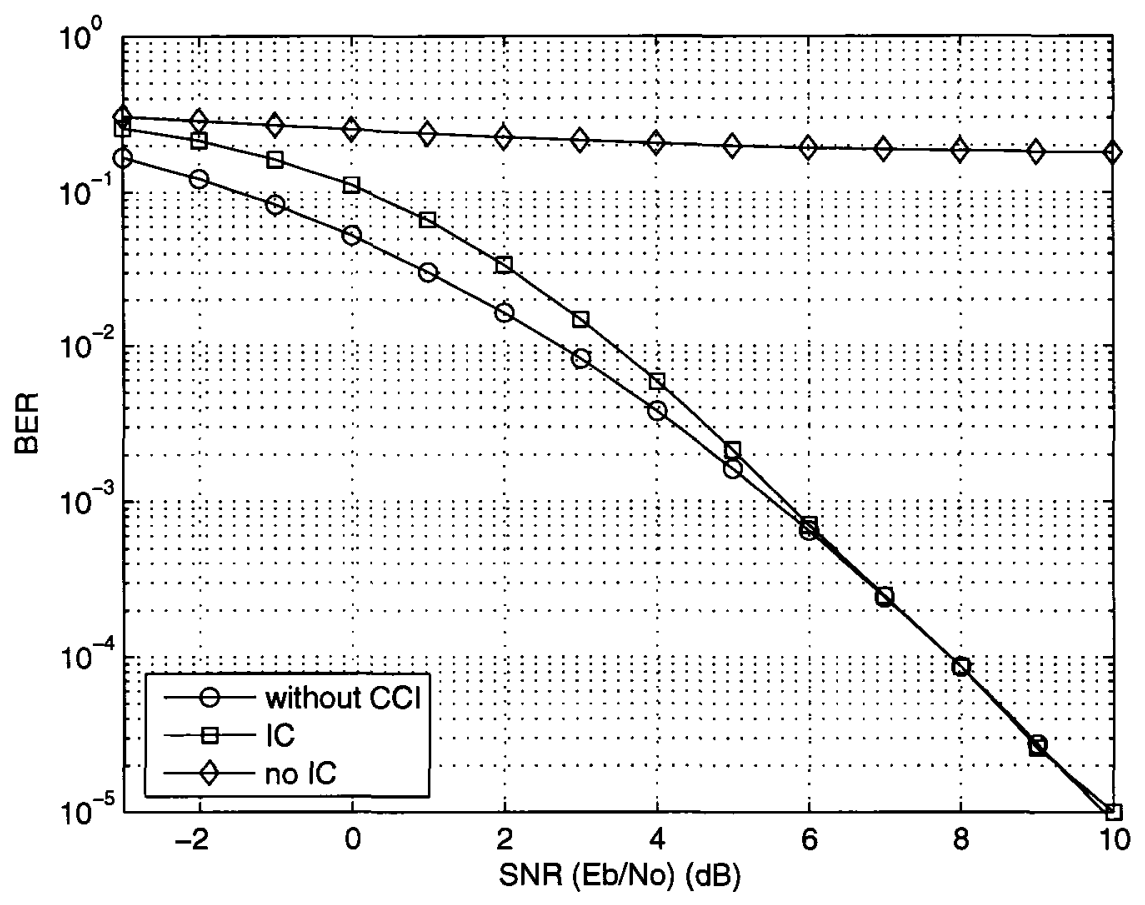

(a) BER performance

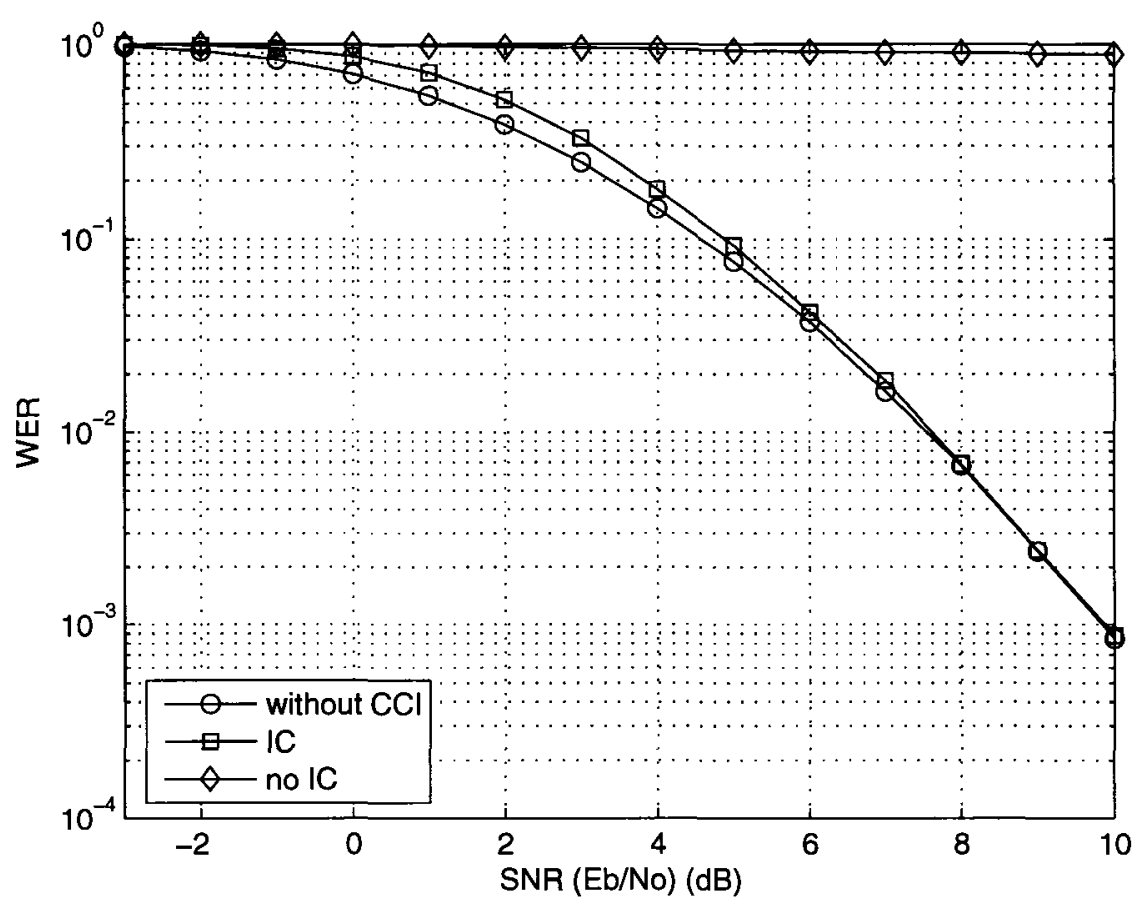

(b) WER performance

Figure 3.12: Performance on SUI-5 channel with $N_{S}=3, N_{A}=2, \Delta=0 \mathrm{~dB}$. 


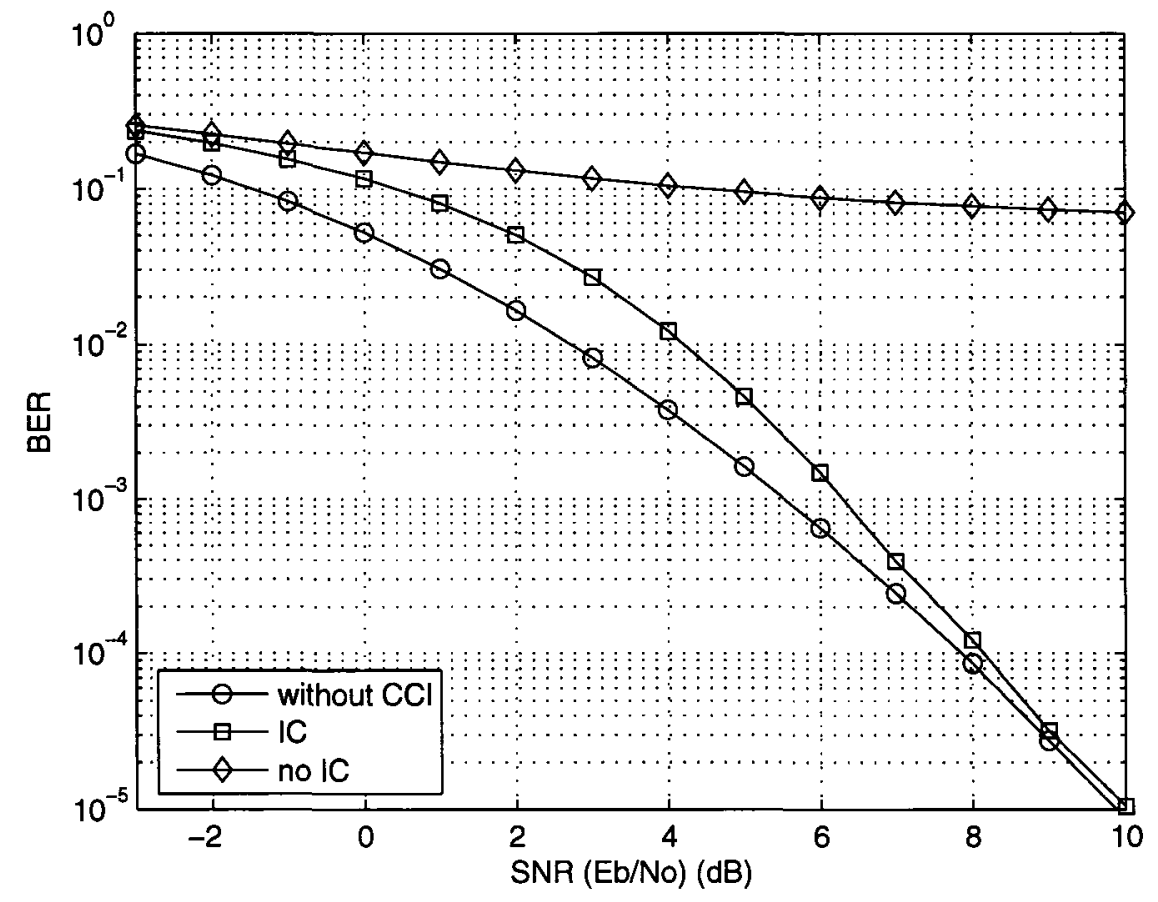

(a) BER performance

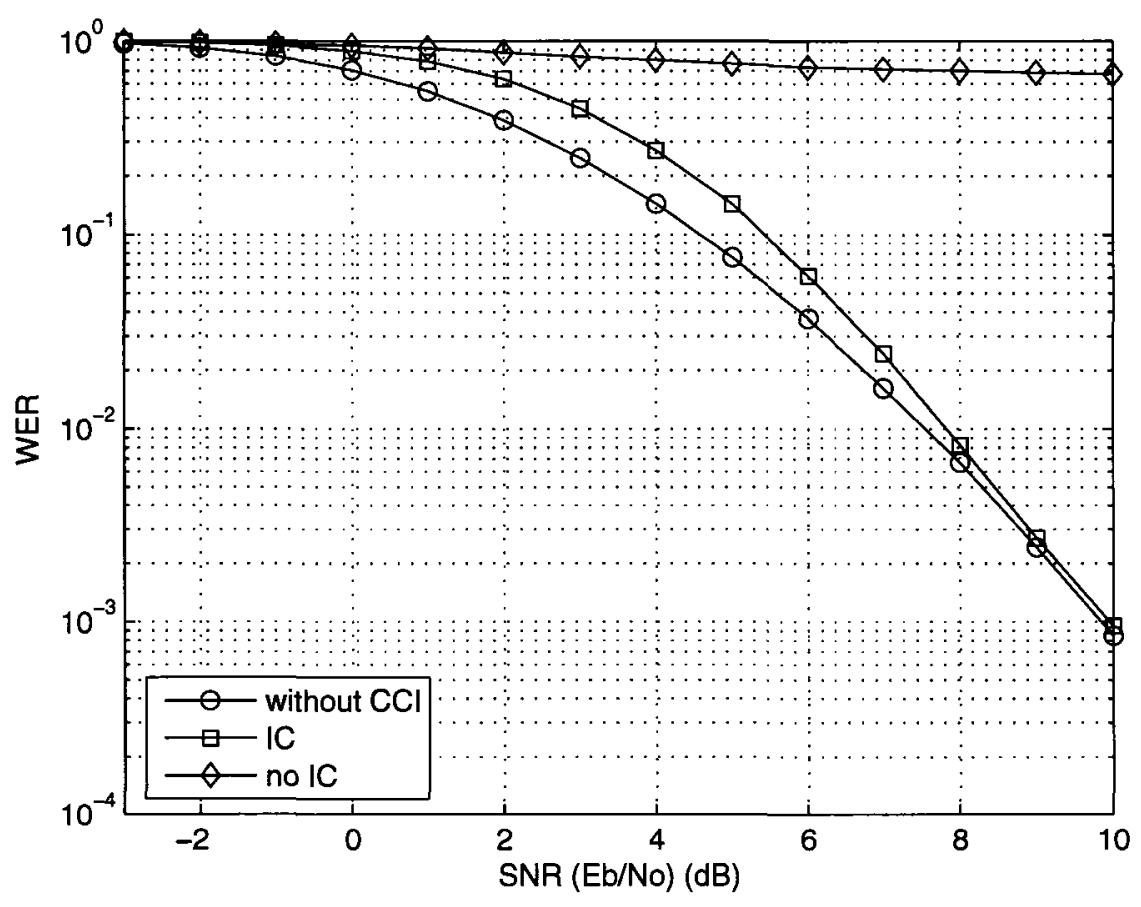

(b) WER performance

Figure 3.13: Performance on SUI-5 channel with $N_{S}=3, N_{A}=2, \Delta=3 \mathrm{~dB}$. 


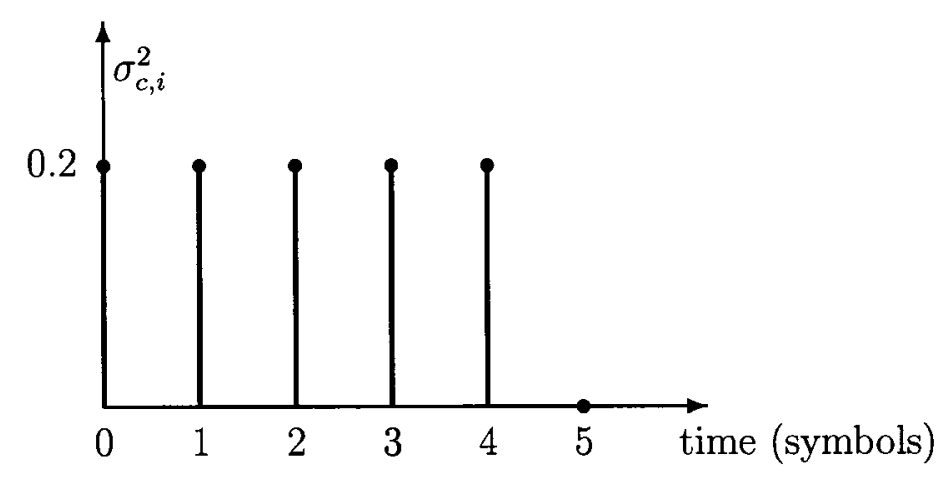

Figure 3.14: 5-tap power delay profile.

\subsection{System Performance for 5-tap Channel}

Figure 3.14 shows the ISI power delay profile of the 5-tap channel. The channel has 5 path components which have equal average power attenuation. Each of the 5 path components experiences independent fading.

\subsubsection{Performance for $N_{S}=2, N_{A}=1$}

Figure 3.15 shows the performance for the $\Delta=0 \mathrm{~dB}$ situation. Since the 5 -tap channel has more ISI components than the SUI-5 channel, the probability that all five ISI components simultaneously experience severe fading is much smaller than the 3-tap SUI-5 channel. As a result, the system performs much better over the 5-tap channel than over the SUI-5 counterpart. From Figure 3.15 and Figure 3.4, we can see that the difference is about $5.5 \mathrm{~dB}$ at a BER of $10^{-5}$.

However, we also notice that the bump in Figure 3.15 is larger than that in Figure 3.4. This is due to the increased error propagation caused by the additional taps. Imagine that all the symbols in a CCI block are decoded correctly except for one. 


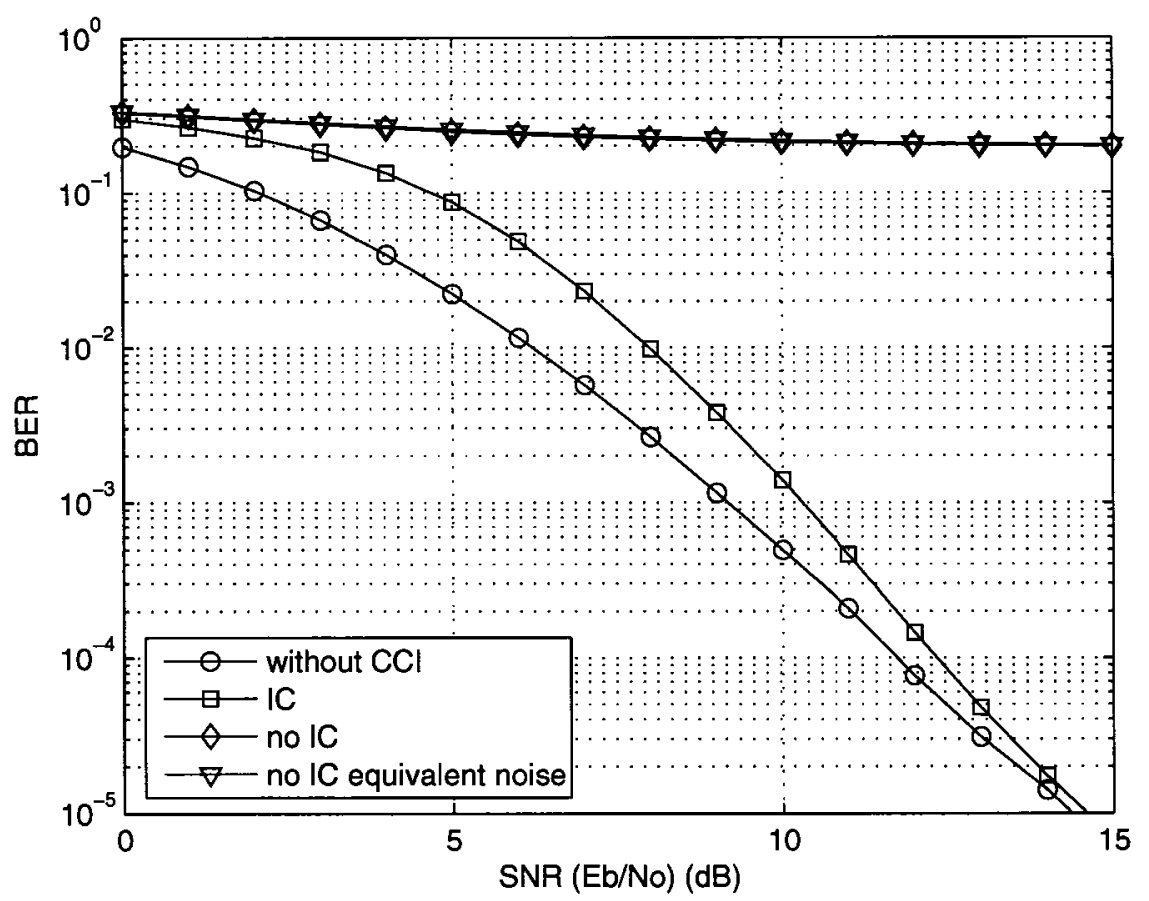

(a) BER performance

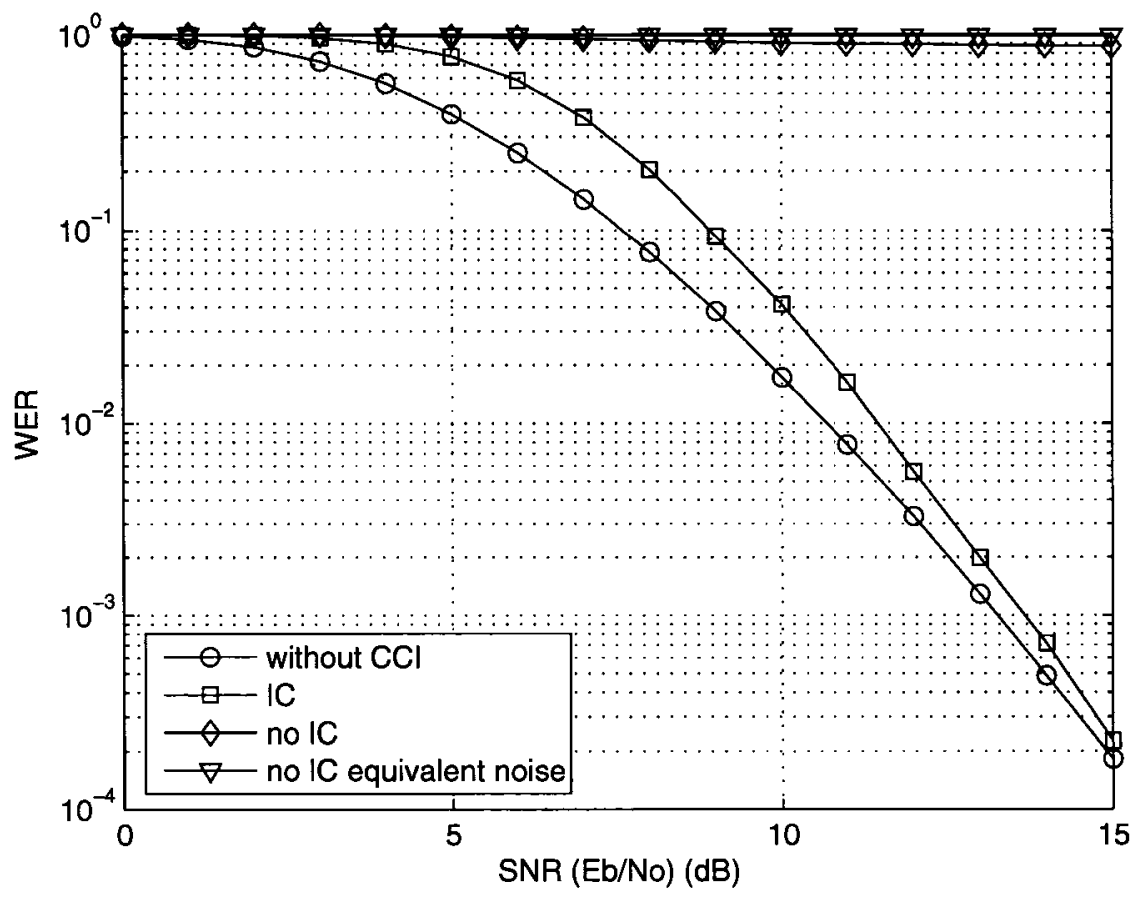

(b) WER performance

Figure 3.15: Performance on 5-tap channel with $N_{S}=2, N_{A}=1, \Delta=0 \mathrm{~dB}$. 
The IC will not be able to cancel the CCI in the five samples that depend on that symbol. Not only that, though, the IC will attempt to subtract the incorrect symbol from those samples, compounding the problem. As a result, five samples in the desired block will remain inflicted with residual CCI, increasing the likelihood that it will be decoded incorrectly, compared to the SUI-5 channel, where only three samples will be inflicted. Nonetheless, the IC is still able to provide a substantial improvement in performance than when the CCI is ignored.

Figure 3.16 shows the performance for the $\Delta=6 \mathrm{~dB}$ situation. We can see the right-shift of the bump if we compare Figure 3.15 and Figure 3.16. We can also see the performance difference between "no IC" and "no IC equivalent noise" in Figure 3.16, which we have seen in Figure 3.5.

\subsubsection{Performance for $N_{S}=2, N_{A}=2$ and $N_{S}=3, N_{A}=2$}

Figure 3.17 shows the performance for the $N_{S}=2, N_{A}=2, \Delta=0 \mathrm{~dB}$ situation, and Figure 3.18 shows the performance for the $N_{S}=3, N_{A}=2, \Delta=0 \mathrm{~dB}$ situation. Similar trends as for the SUI-5 channel are apparent. 


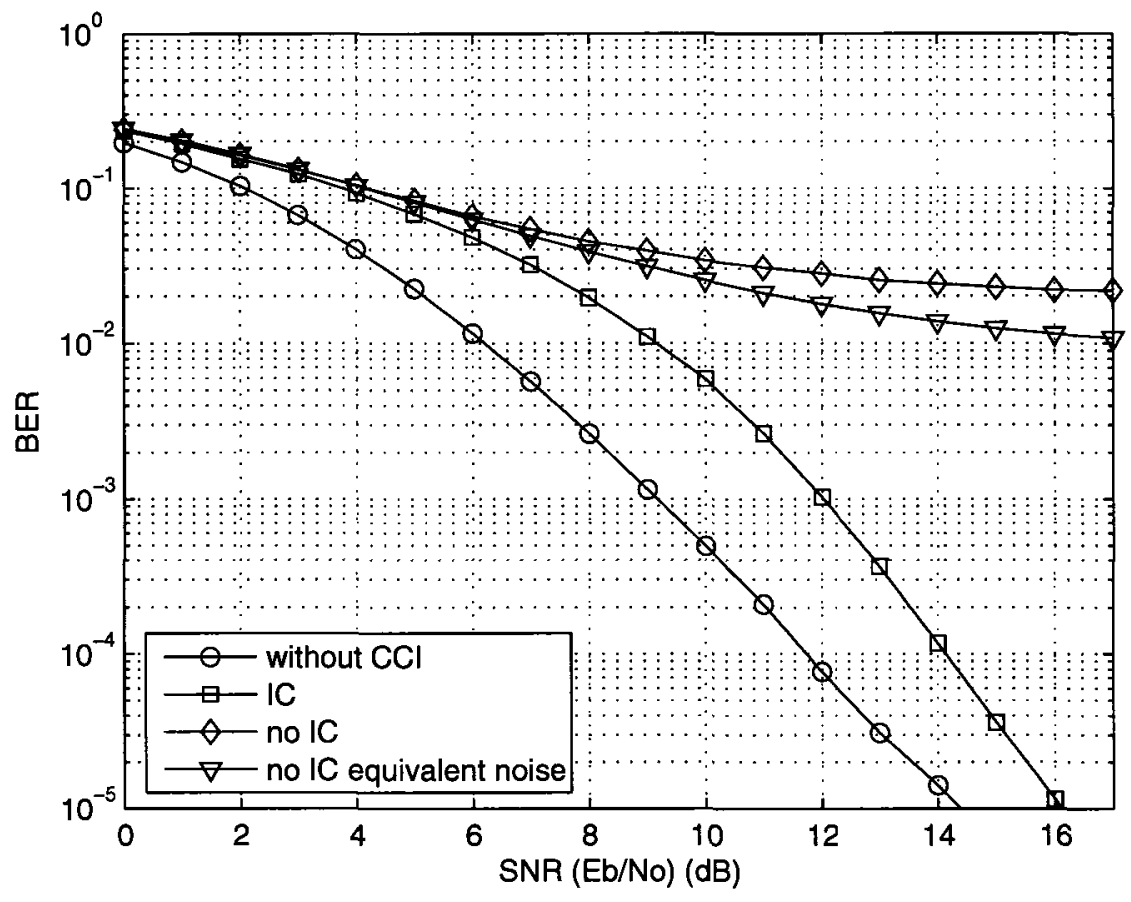

(a) BER performance

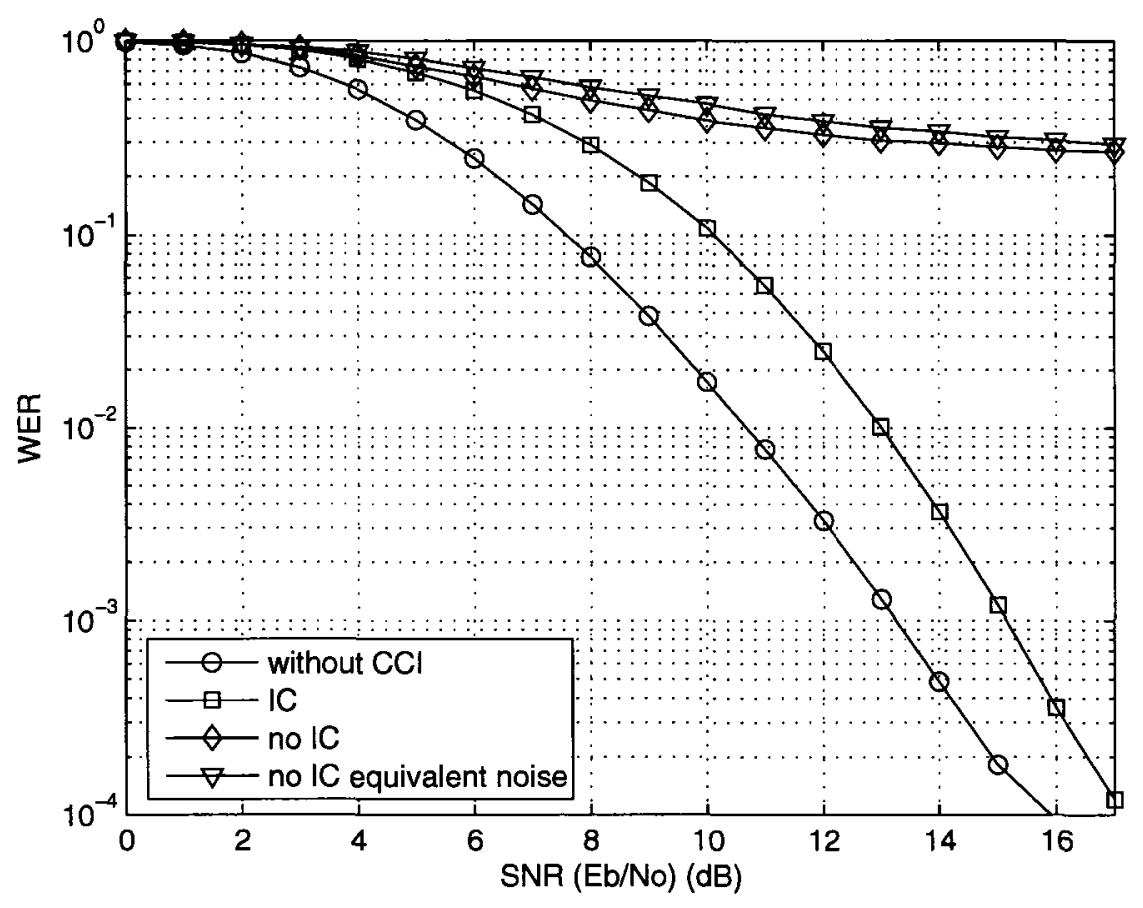

(b) WER performance

Figure 3.16: Performance on 5-tap channel with $N_{S}=2, N_{A}=1, \Delta=6 \mathrm{~dB}$. 


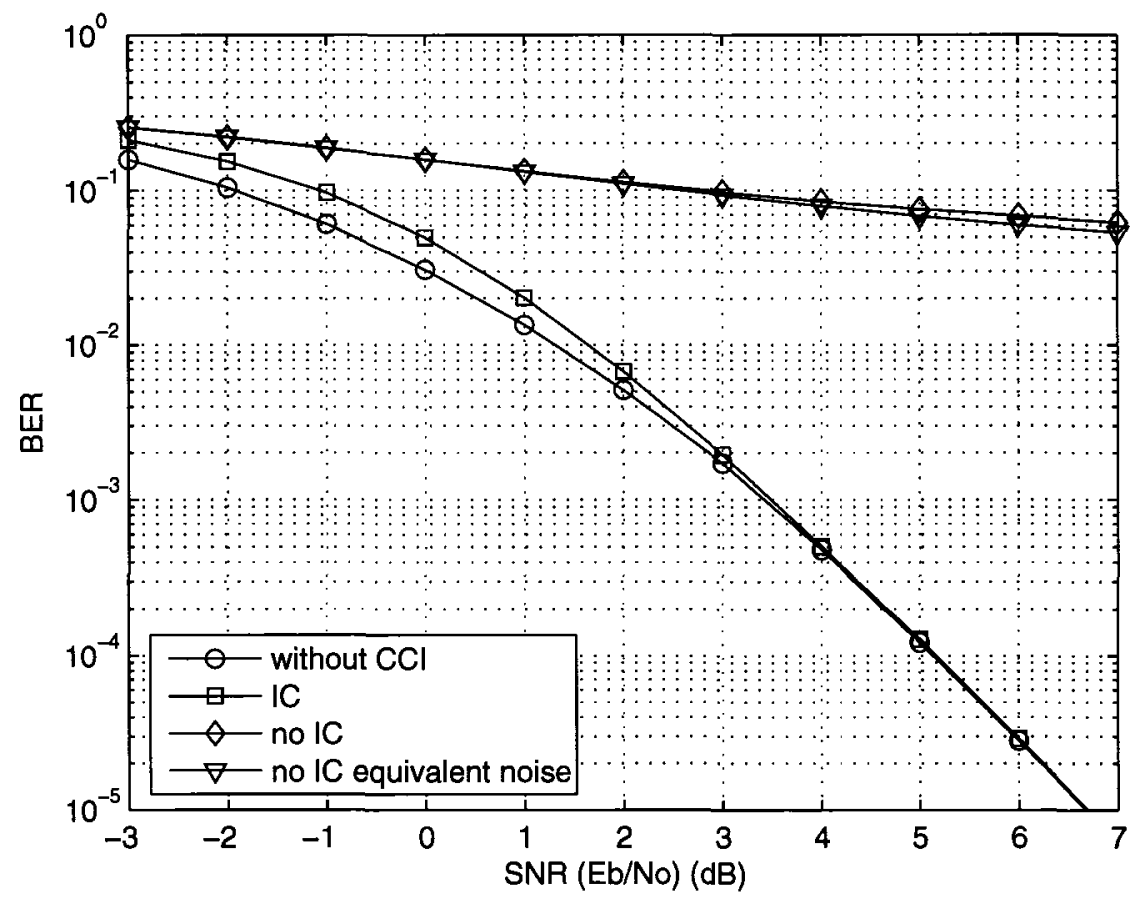

(a) BER performance

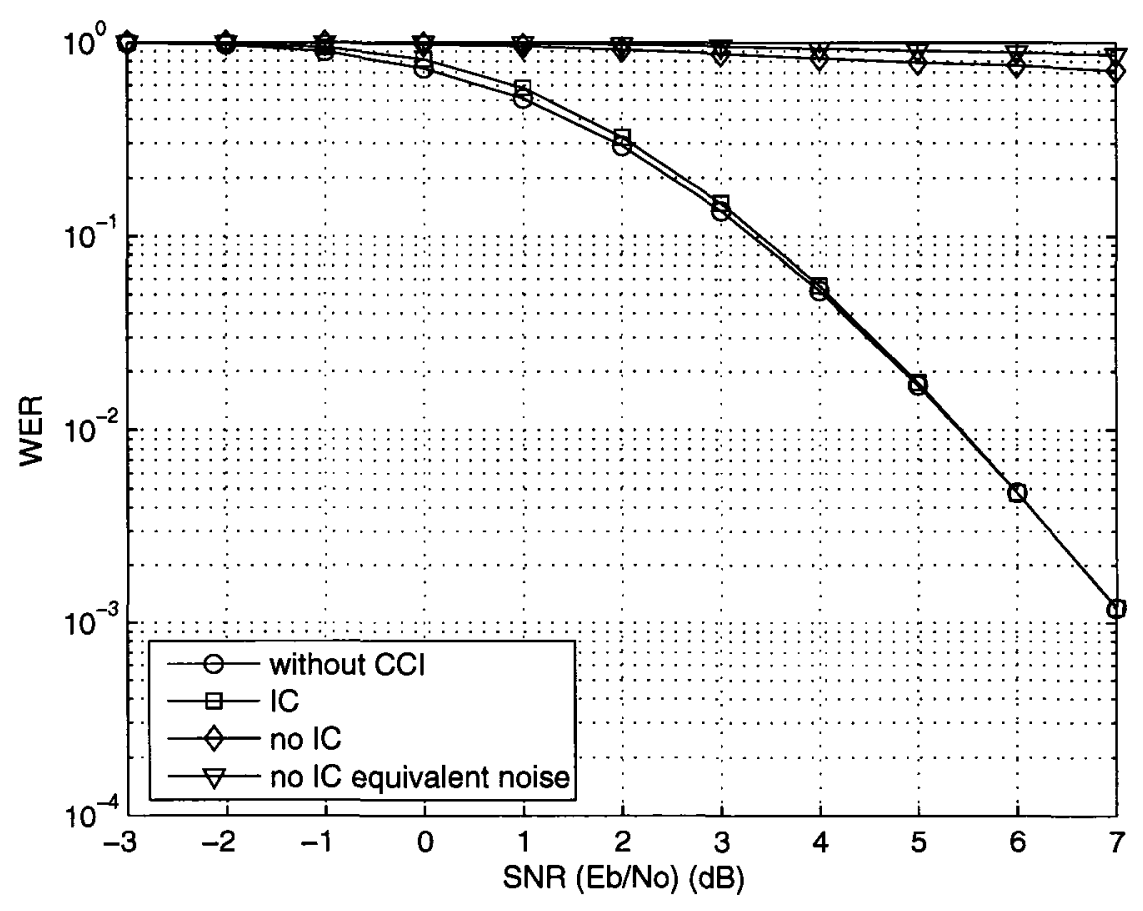

(b) WER performance

Figure 3.17: Performance on 5-tap channel with $N_{S}=2, N_{A}=2, \Delta=0 \mathrm{~dB}$. 


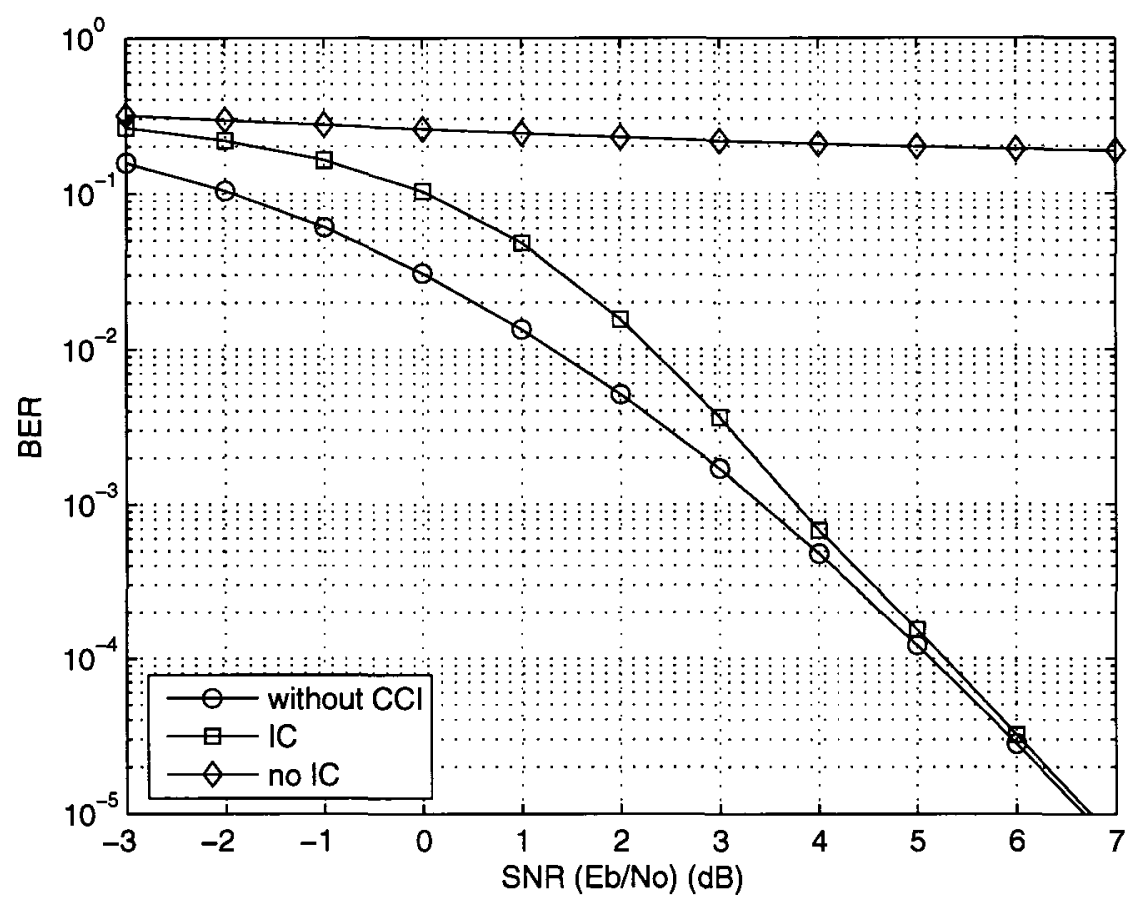

(a) BER performance

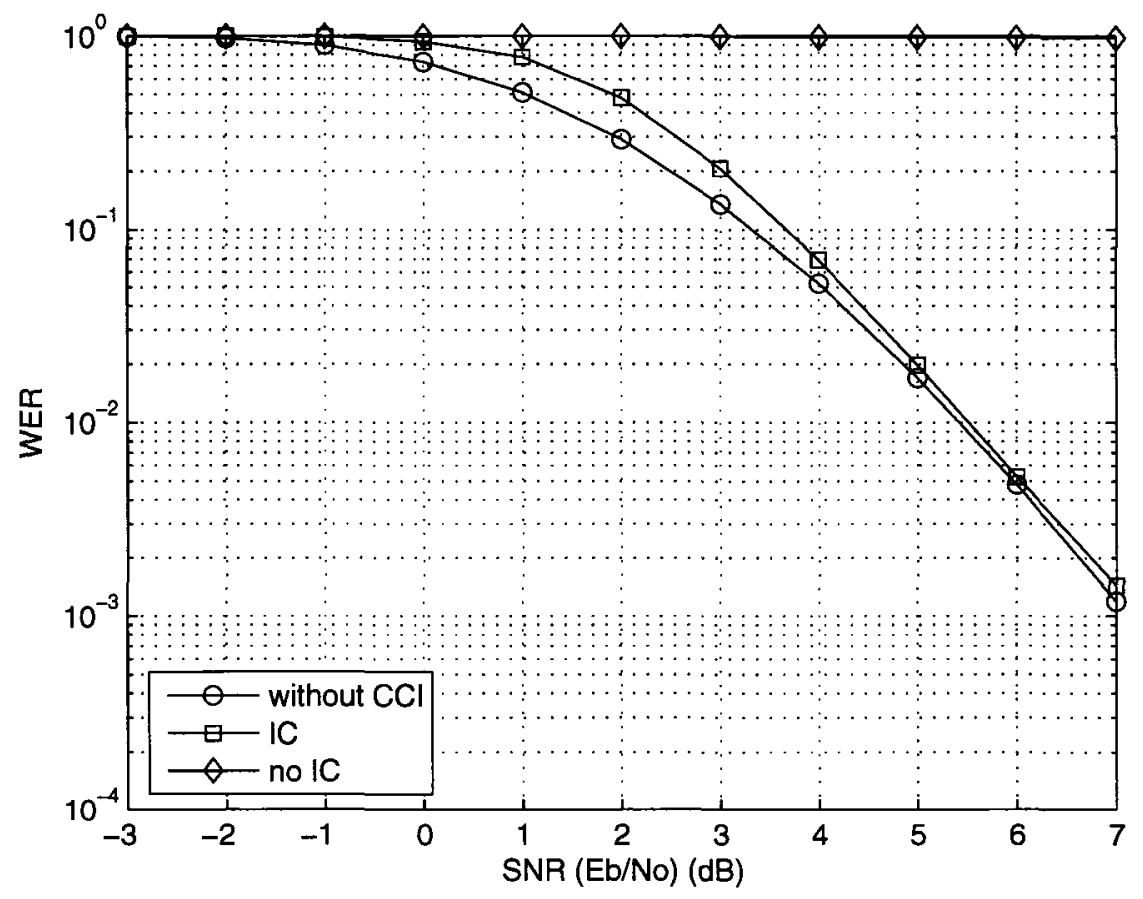

(b) WER performance

Figure 3.18: Performance on 5-tap channel with $N_{S}=3, N_{A}=2, \Delta=0 \mathrm{~dB}$. 


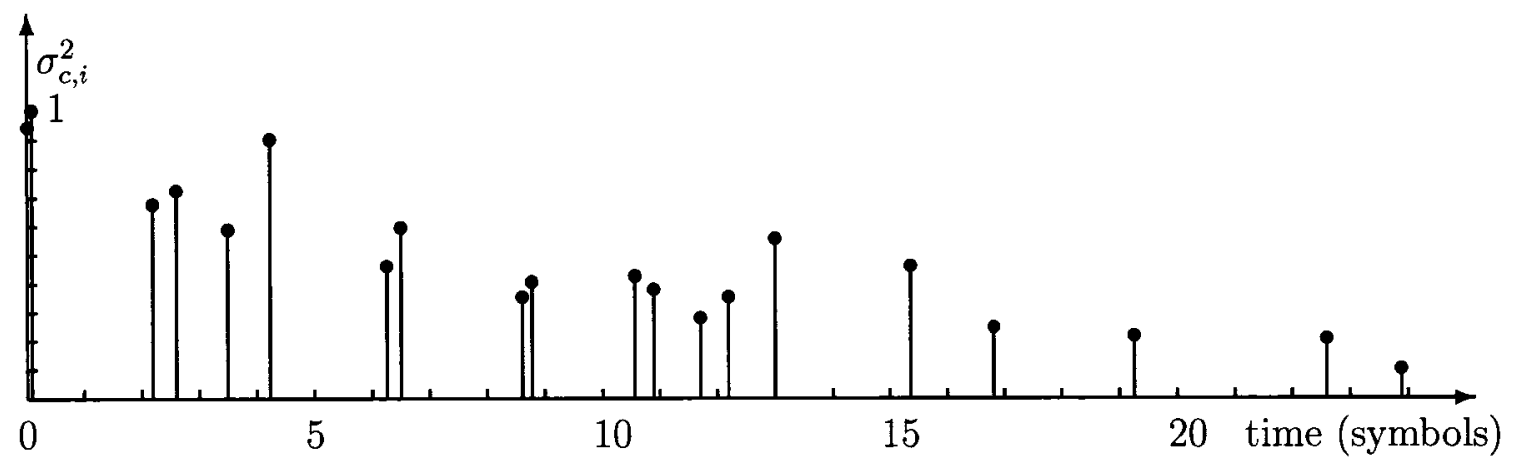

Figure 3.19: Rich Scattering channel power delay profile.

\subsection{System Performance for Rich Scattering Chan- nel}

In this section we explore the system performance in a very rich scattering environment, with a large number of multi-path components. This channel model has 20 components, with path delays (in nano-seconds) of: $0,5,135,160,215,260,385,400$, $530,540,650,670,720,750,800,945,1035,1185,1390$, and 1470 , and the symbol rate is 16.25 MBaud. The path powers in $\mathrm{dB}$ (before normalization) are: $-0.5,0,-3.4$, $-2.8,-4.6,-0.9,-6.7,-4.5,-9.0,-7.8,-7.4,-8.4,-11,-9,-5.1,-6.7,-12.1,-13.2,-13.7$, and -19.8 respectively. Figure 3.19 shows the power delay profile of this channel, which we call the Rich Scattering channel.

Since the intervals between neighboring taps in this model are not integer multiples of the symbol period, we omit the tap random delay to avoid a severe violation of the ISI structure. That is, we use $\tau_{c, i}^{(s)}=\bar{\tau}_{c, i}$. 


\subsubsection{Performance for $N_{S}=2, N_{A}=1$}

Performance for $\Delta=0 \mathrm{~dB}$

Figure 3.20 shows the performance for the $N_{S}=2, N_{A}=1, \Delta=0 \mathrm{~dB}$ case. Compared to the BER performance over the SUI-5 channel as shown in Figure 3.4, the performance over the Rich Scattering channel is much worse at low SNR. However, as the SNR increases, the performance improves rapidly, and is better than for the SUI-5 channel for SNRs greater than about $17 \mathrm{~dB}$. This is because, on one hand, the Rich Scattering channel provides much better frequency diversity than the SUI- 5 channel, so better performance is possible, but, on the other hand, the additional ISI caused by the large number of paths is much harder to equalize, degrading performance, particularly at low SNRs, even in the absence of CCI.

We also notice that the discrepancy between the "without CCI" curve and the "IC" curve is larger than that of the SUI-5 channel and the 5-tap channel. As was noticed with the 5-tap channel, but is more pronounced here, the additional ISI inhibits the ability of the receiver to suppress the CCI.

Performance for $\Delta=12 \mathrm{~dB}$

Figure 3.21 shows the performance for the $N_{S}=2, N_{A}=1, \Delta=12$ case. Obviously, the "no IC" curve is close to the "IC" curve, but far away from "no IC equivalent noise" curve. To better understand the reason of this discrepancy, we add the "no IC equivalent color noise" scheme in this case. The results is shown in Figure 3.22, where "no IC equivalent color noise" means that the white noise whose power is 


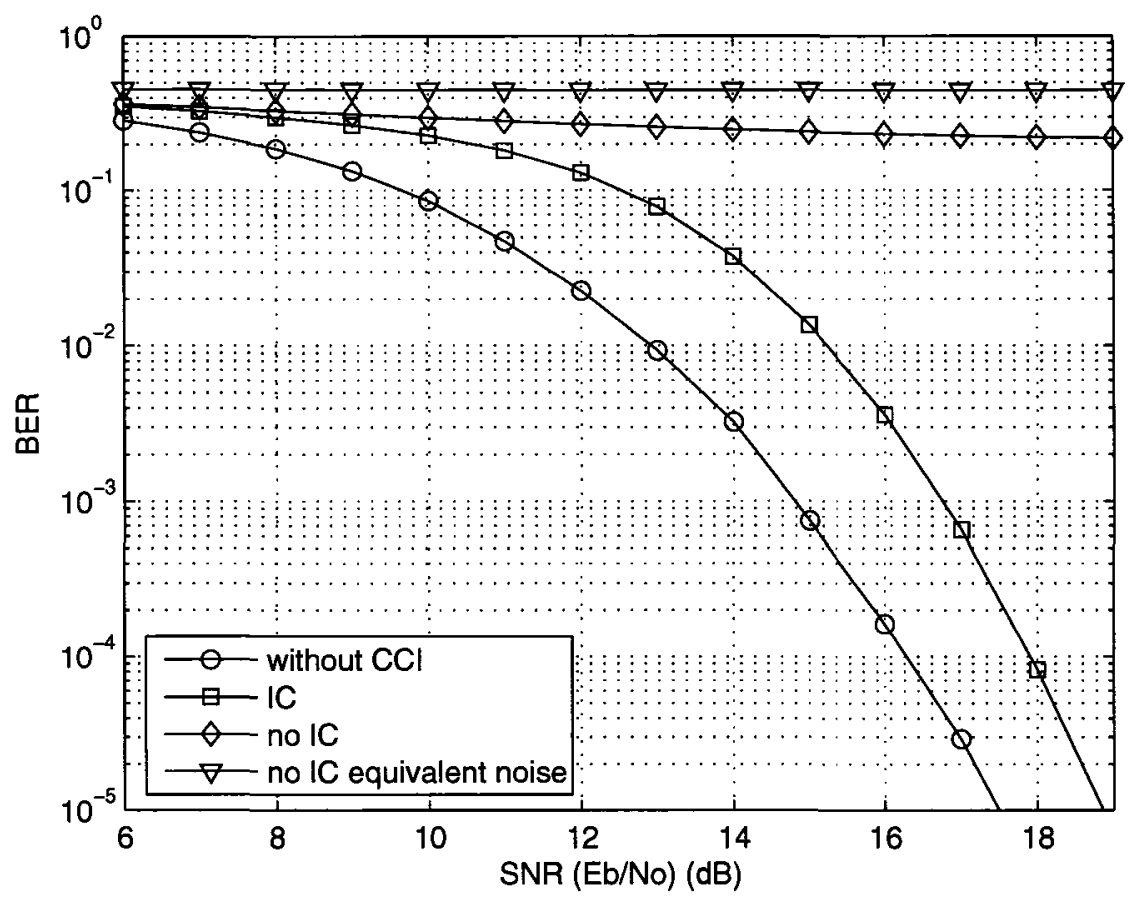

(a) BER performance

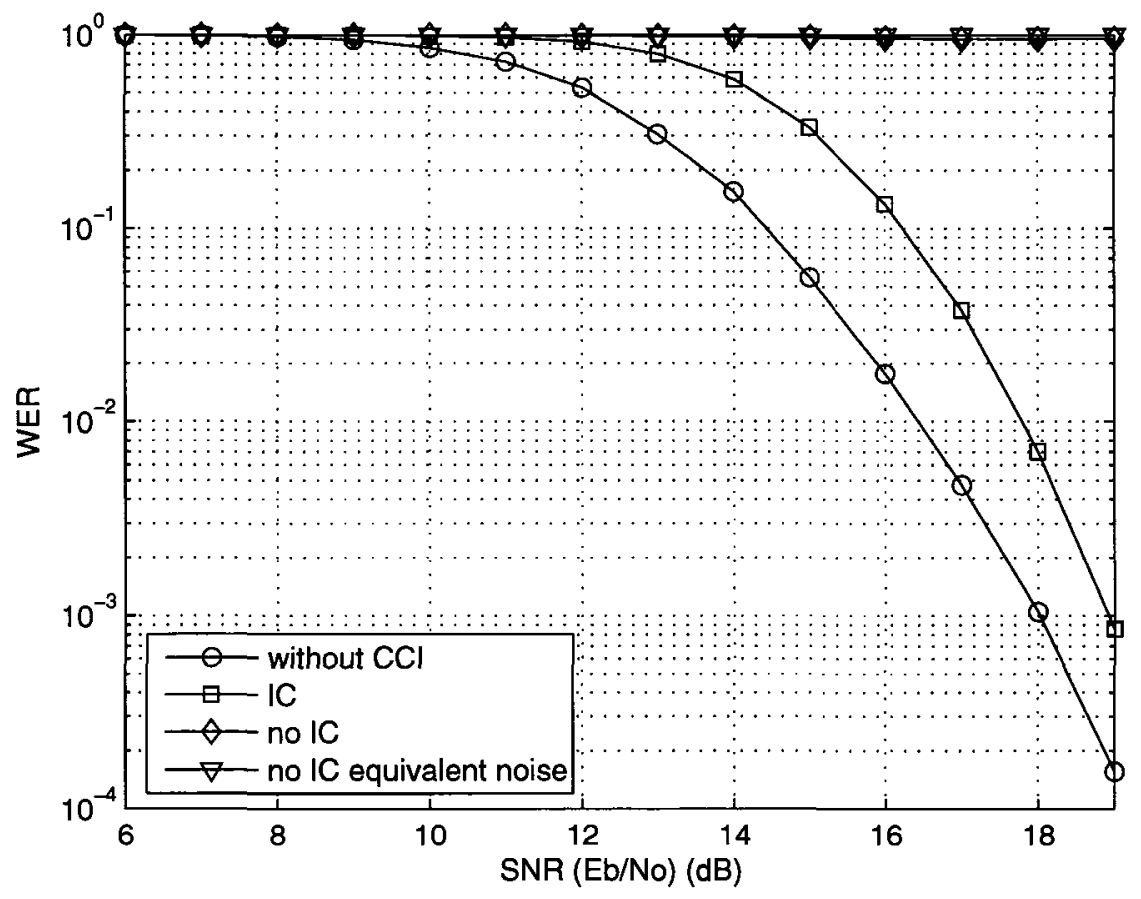

(b) WER performance

Figure 3.20: Performance on Rich Scattering channel with $N_{S}=2, N_{A}=1, \Delta=0$ dB. 


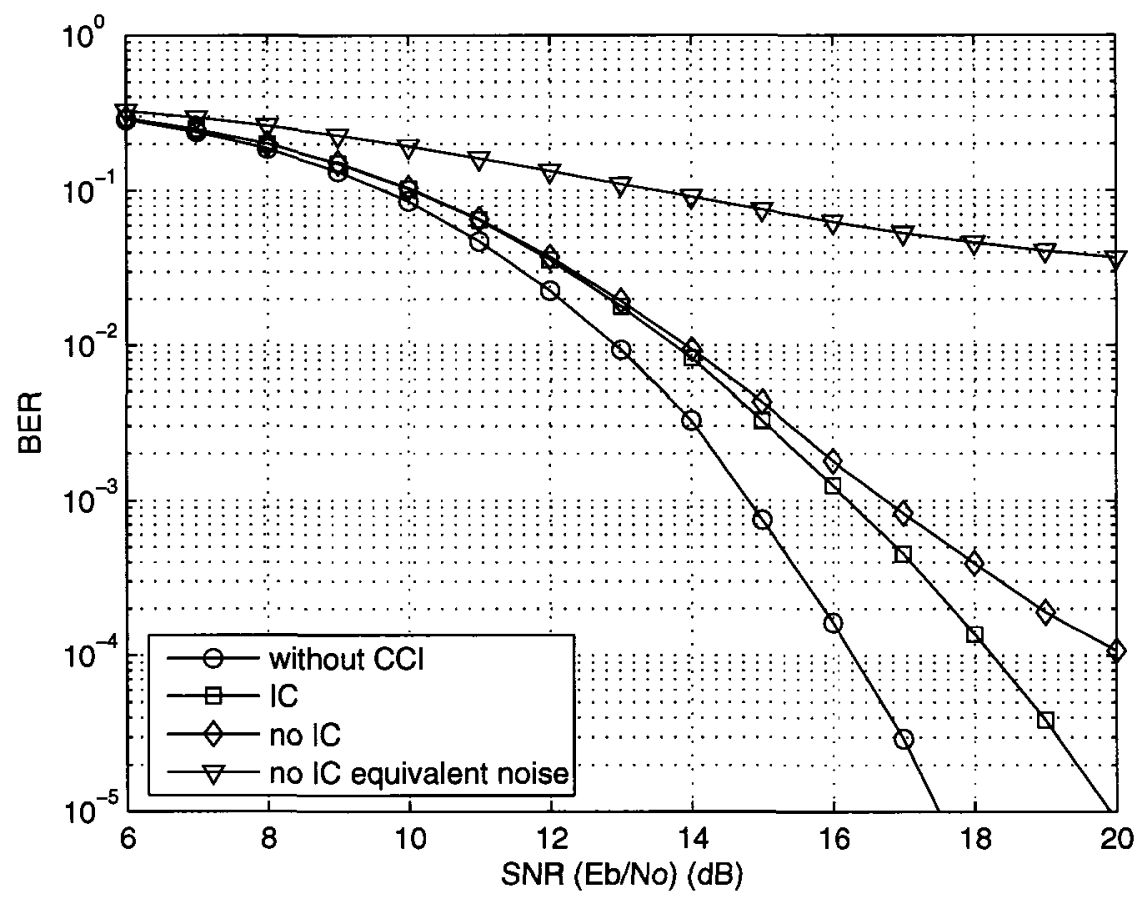

(a) BER performance

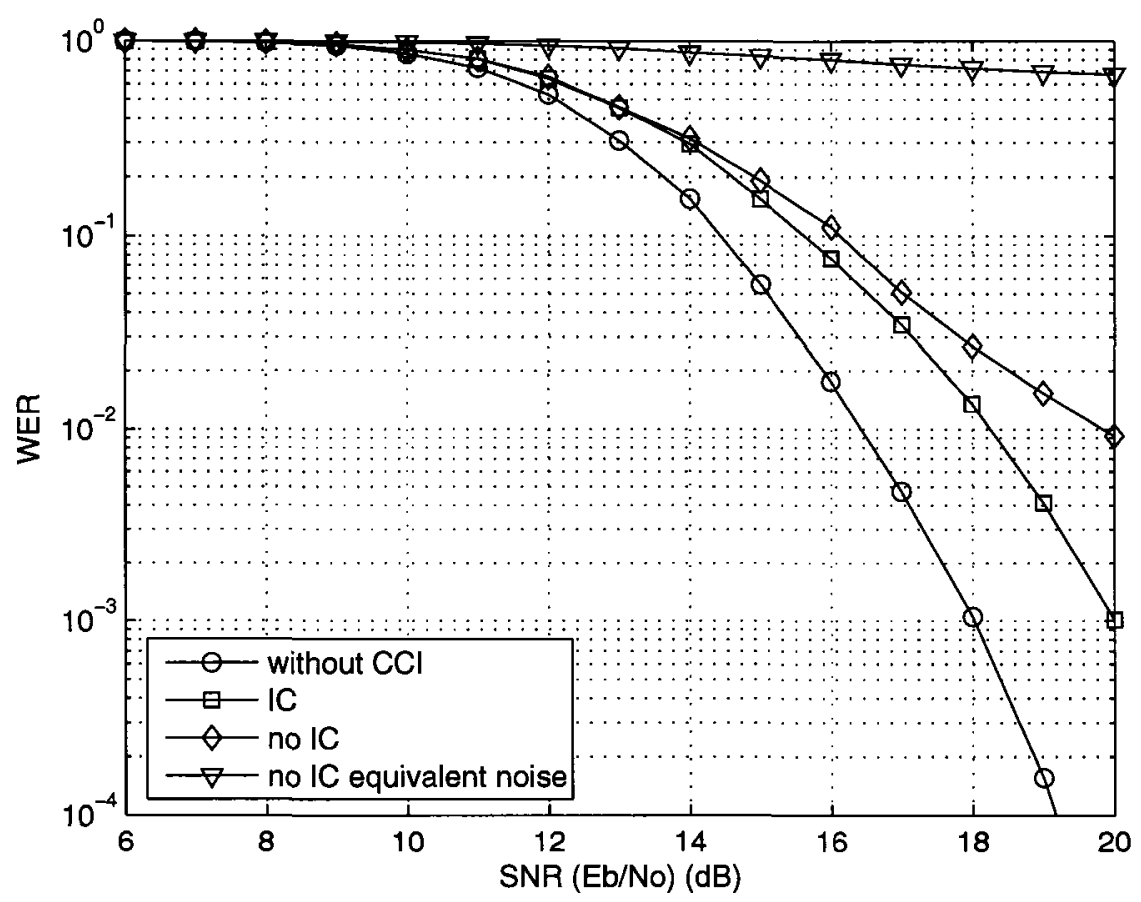

(b) WER performance

Figure 3.21: Performance on Rich Scattering channel with $N_{S}=2, N_{A}=1, \Delta=12$ $\mathrm{dB}$. 


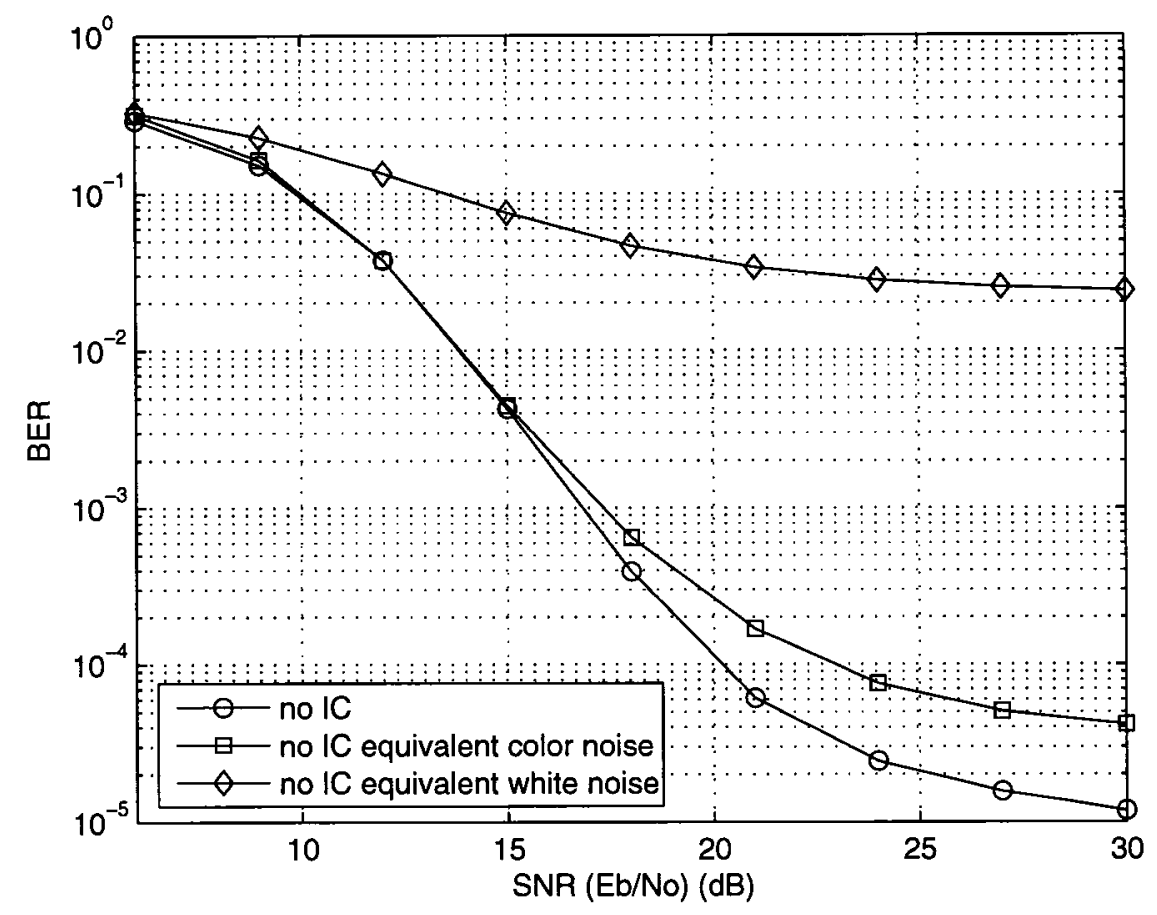

Figure 3.22: BER performance of "no IC" schemes on Rich Scattering channel with $N_{S}=2, N_{A}=1, \Delta=12 \mathrm{~dB}$.

equivalent to the interferer is sent through the channel (including pulse shaping and received filters), and then the consequent narrow-band noise is added to the signal. We can clearly see that the Rich Scattering channel significantly reduces the effect of interference or noise. The reason probably comes from the correlation of multiple ISI taps. As we mentioned before, the application of pulse shape may partially cancel some ISI taps in Figure 3.19 and generate some new ISI taps with smaller amplitude at sampling moments, producing the "picket fence" effect. If $\Delta$ is large, the tap amplitude of the interference is so small that these taps affect decoding much less than the purely white equivalent noise. 


\subsubsection{Performance for $N_{S}=2, N_{A}=2$ and $N_{S}=3, N_{A}=2$}

Figure 3.23 and Figure 3.24 show the performance for $N_{S}=2, N_{A}=2, \Delta=0 \mathrm{~dB}$ and $N_{S}=3, N_{A}=2, \Delta=0 \mathrm{~dB}$ respectively. Similar trends as for the SUI- 5 channel are apparent. In particular, the spatial diversity from the additional receive antenna leads to better overall performance, and better CCI suppression. 


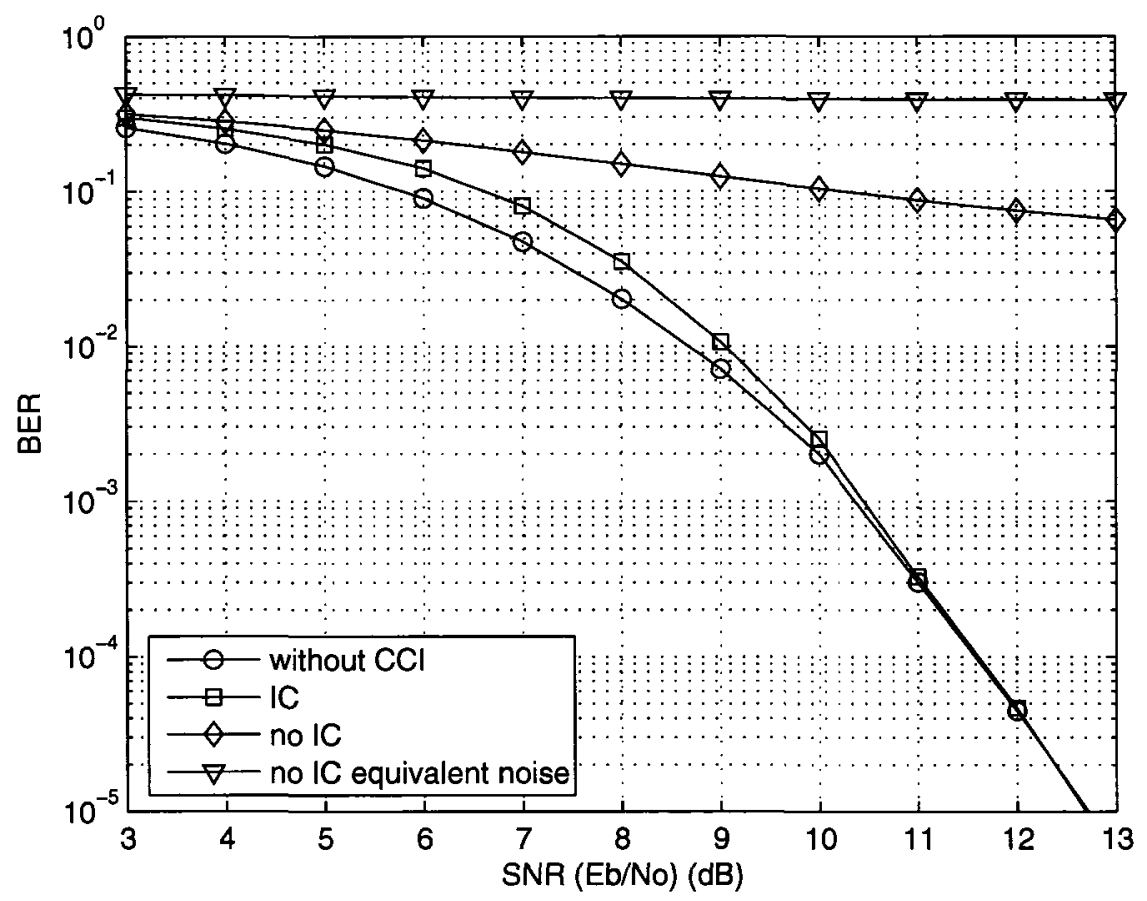

(a) BER performance

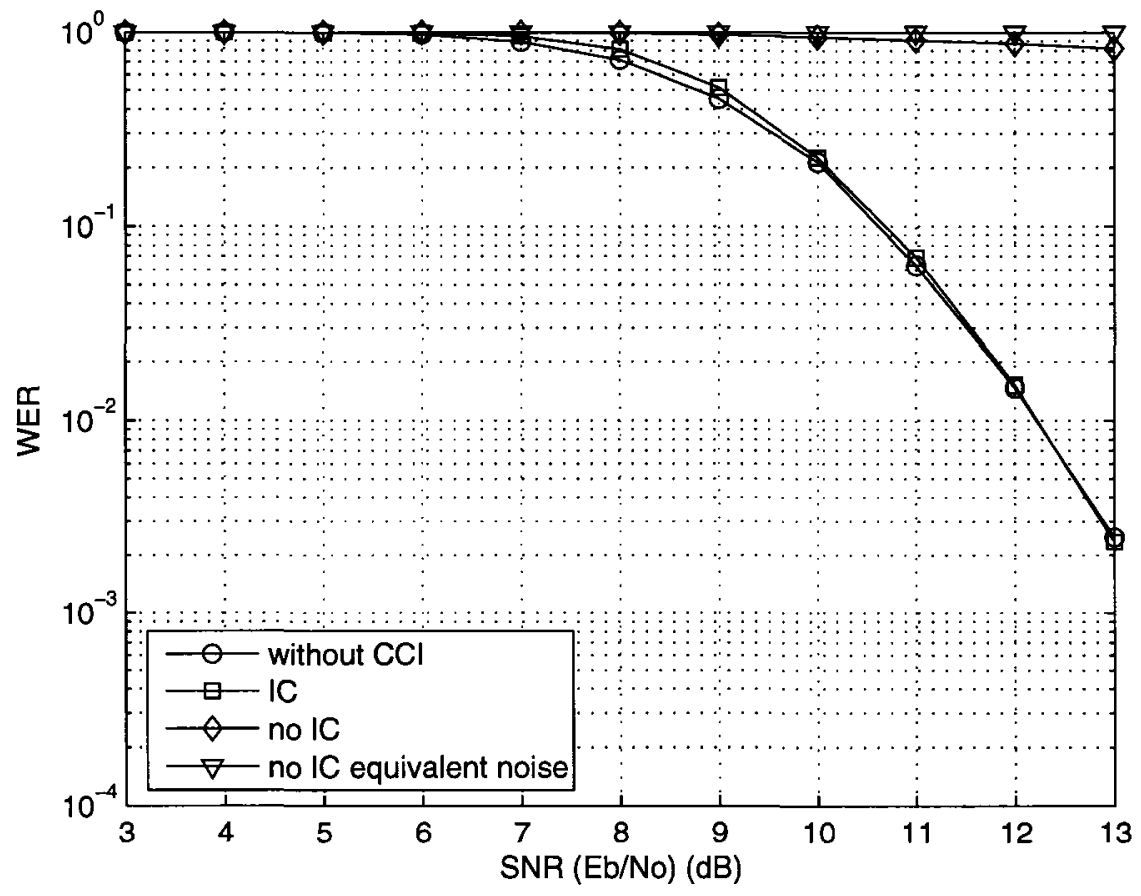

(b) WER performance

Figure 3.23: Performance on Rich Scattering channel with $N_{S}=2, N_{A}=2, \Delta=0$ dB. 


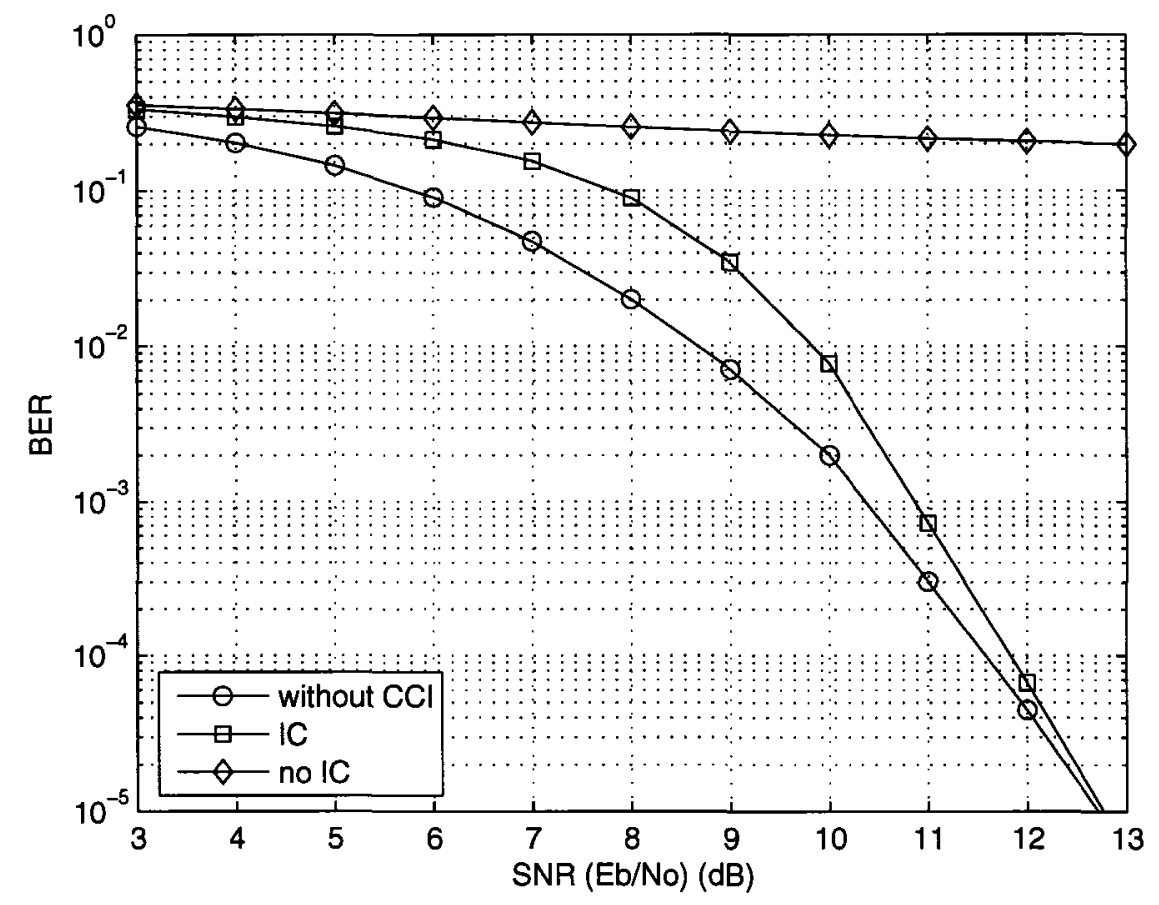

(a) BER performance

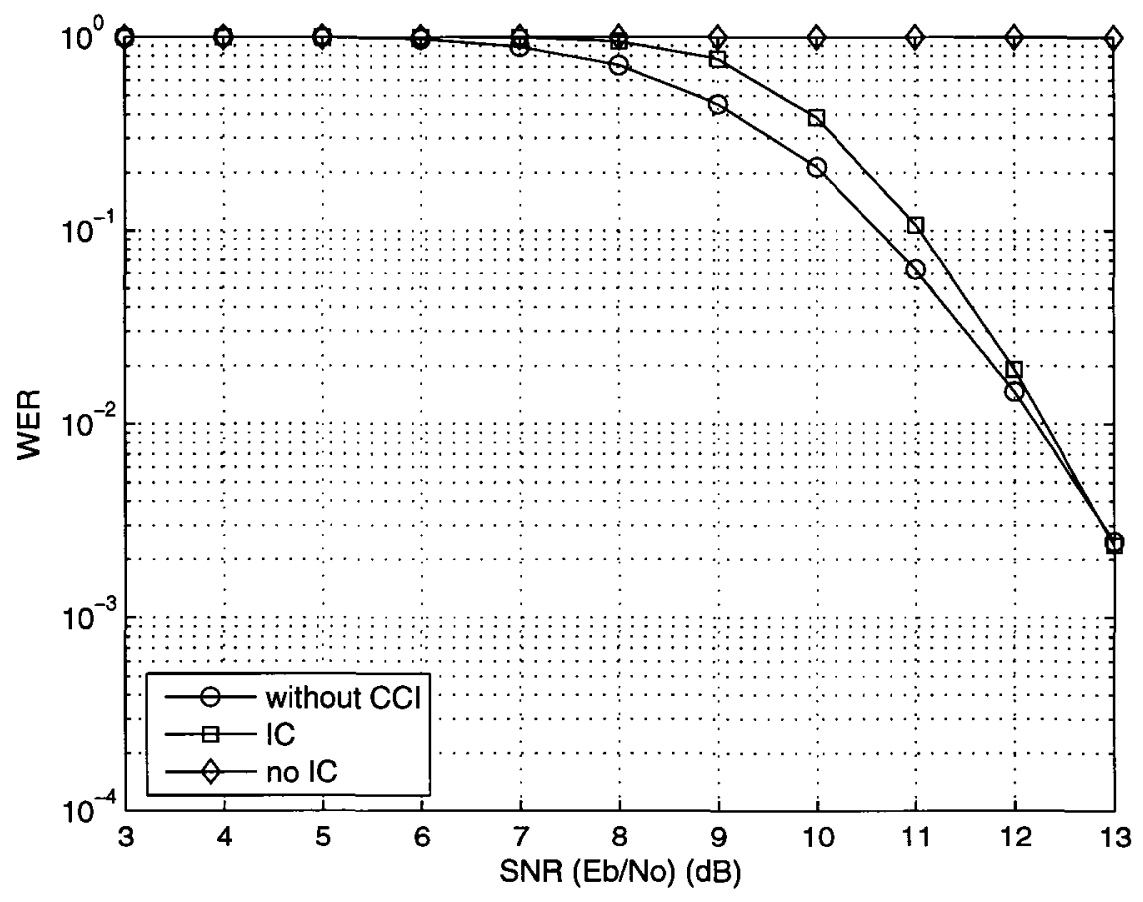

(b) WER performance

Figure 3.24: Performance on Rich Scattering channel with $N_{S}=3, N_{A}=2, \Delta=0$ $\mathrm{dB}$. 


\section{Chapter 4}

\section{Cellular Simulation Results}

The previous chapter has shown us the BER and WER improvement of the asynchronous interference canceler (IC) over the no-IC case. In some cases it is possible to cancel the CCI to a negligible level, while in all cases there is a significant improvement in performance by using the IC. However, we still do not know how much benefit we can obtain from the IC in a practical cellular system. In this chapter, we will use a cellular system model to show the improvement in terms of connection rate, outage rate, outage probability, and system throughput. We compare systems where no IC is used ("no IC"), where the asynchronous IC described in Chapter 2 is used ("IC"), and a hypothetical system that is capable of perfectly canceling the strongest (primary) interferer ("perfect IC"). 


\subsection{Cellular Model Description}

In our cellular model, 37 cells form a 3-tier cellular system. Several different frequency reuse patterns and cell sectoring schemes are considered and compared, and each sector has one transmit antenna and each mobile station (MS) has only one receive antenna. We consider the moderate SUI-5 channel as the radio propagation model. Most system parameters can be found in Table 4.1.

In our model, the transmit power of any BS antenna $\left(P_{t}\right)$ is $43 \mathrm{dBm}$. The antenna gain of the MS receive antenna $\left(G_{r}\right)$ is $-1 \mathrm{dBi}$, and, when sectoring is used, the antenna gain of the transmit antenna (in $\mathrm{dB}$ ) is

$$
G_{t}(\theta)=15-\min \left[12\left(\frac{\theta}{\theta_{3 \mathrm{~dB}}}\right)^{2}, A_{\max }\right],
$$

where $\theta$ is the angle of departure (in degrees) to the MS relative to the antenna boresight, $\theta_{3 \mathrm{~dB}}$ is the $3 \mathrm{~dB}$ beamwidth (in degrees) at which the signal power is half of its maximal power, and $A_{\max }=20 \mathrm{~dB}$ is the maximum attenuation of the antenna. If there are 3 sectors in a cell, $\theta_{3 \mathrm{~dB}}=70^{\circ}$; if there are 6 sectors in a cell, $\theta_{3 \mathrm{~dB}}=35^{\circ}$. Figure 4.1 shows the antenna pattern of these two cases. When sectoring is not used, the antenna gain is fixed at $G_{t}(\theta)=15 \mathrm{~dB}$, which does not depend on the angle of departure.

The path loss between a BS antenna and a MS antenna $\left(P_{l}\right.$ in $\left.\mathrm{dB}\right)$ is a function of their separation distance $d$ :

$$
P_{l}(d)=\left\{\begin{array}{cl}
10 \rho \log (35) & \text { if } d \leq 35 \text { meters } \\
10 \rho \log (d) & \text { if } d>35 \text { meters }
\end{array},\right.
$$




\begin{tabular}{|c|c|c|}
\hline Parameter & Value & Remarks \\
\hline Number of cells & 37 & 3-tier cellular layout \\
\hline Sectors per cell & 1,3 , or 6 & \\
\hline $\begin{array}{l}\text { Propagation model (BS } \\
\text { Ant } \mathrm{Ht}=32 \mathrm{~m} \text {, MS Ant } \\
\mathrm{Ht}=1.5 \mathrm{~m} \text { ) }\end{array}$ & $\begin{array}{l}31.5+35 \log (d) \mathrm{dB}, d \text { in } \\
\text { meters }\end{array}$ & $\begin{array}{l}\text { the modified Hata urban } \\
\text { (COST 231) model at } 2 \\
\text { GHz, minimum } 35 \text { meter } \\
\text { separation between BS } \\
\text { and MS }\end{array}$ \\
\hline BS site-to-site distance & $2500 \mathrm{~m}$ & $\begin{array}{l}\text { distance between two ad- } \\
\text { jacent base stations }\end{array}$ \\
\hline Log-normal shadowing & $\begin{array}{l}\text { standard deviation }=6 \\
\mathrm{~dB}\end{array}$ & $\begin{array}{l}\text { different antennas of the } \\
\text { same BS have identical } \\
\text { shadowing }\end{array}$ \\
\hline MS noise figure & $10 \mathrm{~dB}$ & \\
\hline Noise power $\left(P_{N}\right)$ & $5 \times 10^{-11} \mathrm{~mW}$ & \\
\hline Carrier frequency & $2 \mathrm{GHz}$ & \\
\hline$-3 \mathrm{~dB}$ beamwidth & $\begin{array}{l}\text { 1-sector: Omni } \\
\text { 3-sector: } 70 \text { degrees } \\
\text { 6-sector: } 35 \text { degrees }\end{array}$ & \\
\hline $\begin{array}{l}\text { Antenna pattern } \\
\text { maximum attenuation }\end{array}$ & $\begin{array}{l}\text { 3-sector: } 20 \mathrm{~dB} \\
\text { 6-sector: } 20 \mathrm{~dB}\end{array}$ & \\
\hline $\begin{array}{l}\text { Antenna orientation } \\
\text { (main lobe bearing) }\end{array}$ & $\begin{array}{l}\text { 3-sector: } 0 \text { degree } \\
\text { 6-sector: } 30 \text { degrees }\end{array}$ & $\begin{array}{l}\text { 3-sector: to the side } \\
6 \text {-sector: to the corner }\end{array}$ \\
\hline $\begin{array}{l}\text { BS antenna gain with ca- } \\
\text { ble loss }\end{array}$ & $15 \mathrm{~dB}$ & \\
\hline MS antenna gain & $-1 \mathrm{dBi}$ & \\
\hline BS transmit power & $43 \mathrm{dBm}$ & $20 \mathrm{~W}$ \\
\hline
\end{tabular}

Table 4.1: Cellular system parameters. 


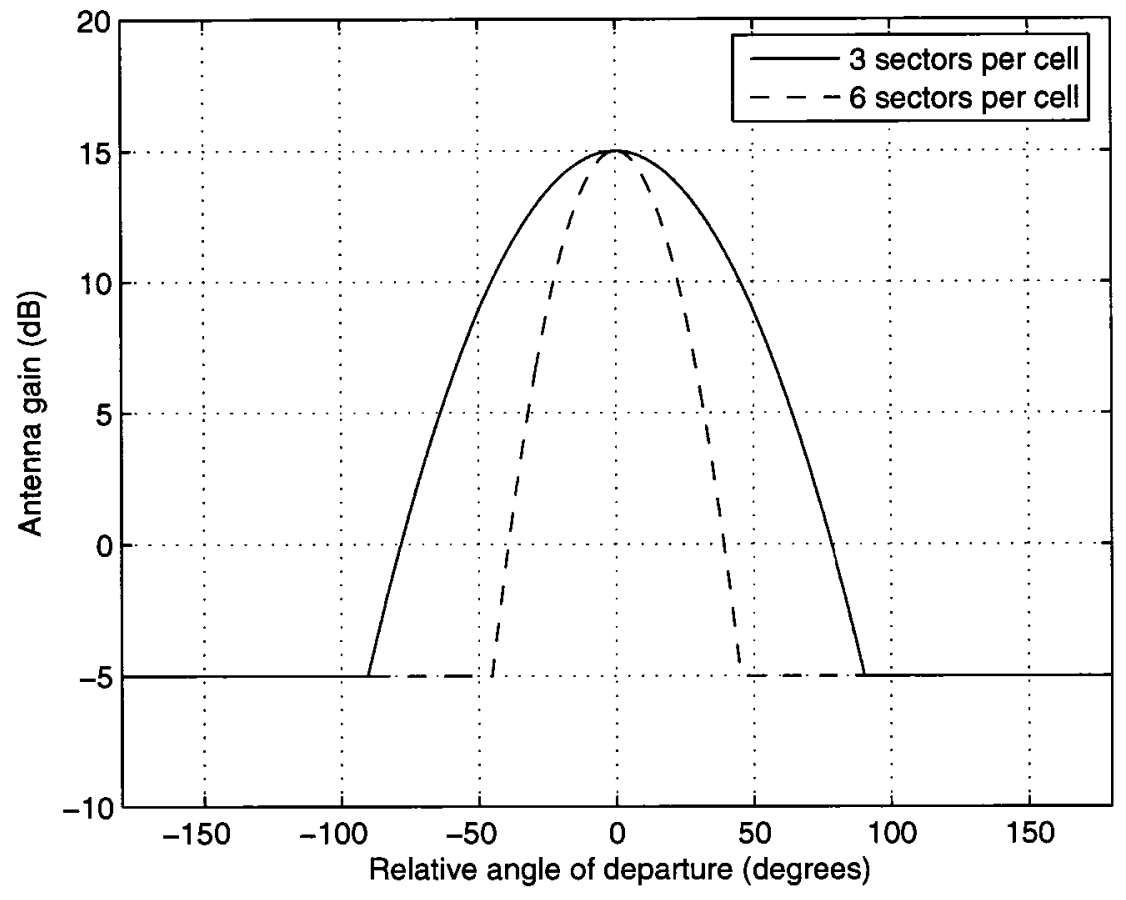

Figure 4.1: Transmit antenna gain for sectoring.

where $\rho$ is the path loss exponent. For the following simulations, we use $\rho=3.5$. To model the random shadowing effects which occur over a large number of measurement locations with the same transmitter-receiver distance, we use log-normal shadowing, so the shadowing $\left(S_{h}\right.$ in $\left.\mathrm{dB}\right)$ is modeled as a zero-mean Gaussian random variable with standard deviation $\sigma_{s}=6 \mathrm{~dB}$. Since the frequency-selective fading effect has been incorporated in the iterative receiver channel model, we omit it here in the cellular model.

The received signal power (in $\mathrm{dBm}$ ) can be expressed as

$$
P_{r}=P_{t}+G_{t}(\theta)+G_{r}-P_{l}(d)+S_{h}
$$

Note that each signal is transmitted with the same transmit power $\left(P_{t}\right)$ and, when 
sectoring is used, all signals from the different sectors of the same cell will have the same path loss $\left(P_{l}\right)$ and shadowing $\left(S_{h}\right)$. Each signal will be subjected to a different transmit antenna gain (due to the different angle of departure), but the same receive antenna gain (since the receive antenna is isotropic).

A MS is potentially able to receive its signal from any sector of any BS, and choose the "best" server. This is done by first calculating the received signal power from each of the sectors, according to Equation 4.3. For all the signals using the same frequency, the base station providing the strongest signal would be used as the server, and all other signals would be interferers. From Chapter 3 we know that with a single receive antenna we are able to cancel a single interferer. We refer to the strongest interferer (the one with the highest power) as the primary interferer. The MS is able to process the desired signal, and the primary interferer that is to be canceled. Other interferers are treated as AWGN with equivalent power and no attempt is made to cancel them. If we let $P_{S}$ denote the power of the desired signal, $P_{N}$ denote the power of the AWGN, $P_{I}$ denote the power of the primary interferer, and $P_{O}$ be the total combined power of all the other interferers, then, in the following, we define the signal-to-noise ratio as

$$
\mathrm{SNR}=\frac{P_{S}}{P_{O}+P_{N}}
$$

the signal-to-interference-plus-noise ratio as

$$
\mathrm{SNR}=\frac{P_{S}}{P_{I}+P_{O}+P_{N}}
$$

and the signal-to-interference ratio as

$$
\Delta=\operatorname{SIR}=\frac{P_{S}}{P_{I}}
$$




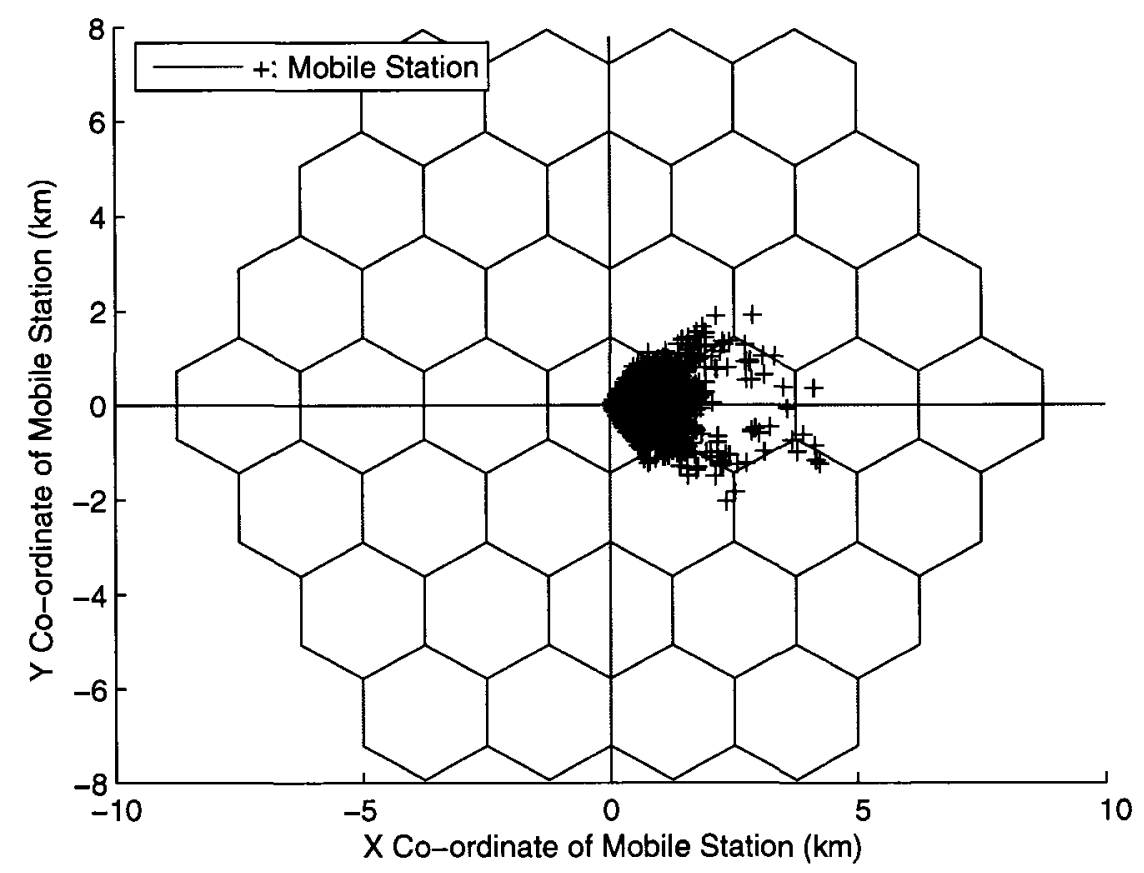

Figure 4.2: Cellular layout.

Once the SNR and SINR has been calculated for each frequency group, the serving sector (or cell) can be selected. For the "no IC" case, the frequency group with the highest SINR is chosen, and the serving sector is the one transmitting the strongest signal in that group. For the "IC" and "perfect IC" cases, where we assume the primary interferer can be canceled, we choose the frequency group with the highest SNR. Thus, for the same user, different serving sectors may be chosen for the "no IC" case and the "IC" cases.

Figure 4.2 shows an example of the cellular system layout. In this example, there are 3 sectors in a cell. The central cell is denoted as cell 0 , and the east-bearing (antenna orientation $=0$ degree) cell is called sector 0 . Each MS is served by the sector with the highest SINR (or SNR). The figure shows with a "+" every MS whose 
serving sector is sector 0 of cell 0 .

\subsection{Simulation Results of Connection Rates}

To measure the system performance, we consider the connection rate, which is the average realized data rate for a user, assuming that incorrectly received codewords are detected (using a embedded cyclic redundancy check (CRC) code) and retransmitted. The connection rate is

$$
C_{R}=\eta_{\mathrm{nom}}(1-\mathrm{WER})
$$

where $\eta_{\text {nom }}$ is the nominal throughput (in message bits per channel use) and WER is the word error rate for the SNR and SIR $(\Delta)$ experienced by the user, as presented in Chapter 3. For QPSK with a rate $1 / 2$ convolutional code, the nominal throughput is 1 bit per channel use (where the slight overhead due to trellis termination and the CRC code has been ignored).

Table 4.2 shows the connection rate of various SNR and $\Delta$, and Figure 4.3 shows a 3-D graph of this connection rate. Several connection rate curves of different $\Delta$ are also shown in a 2-D form in Figure 4.4. Recall that $\Delta$ is the difference in $\mathrm{dB}$ between the signal and the primary interferer. In this chapter, the "perfect IC" results correspond to a hypothetical ideal system that can perfectly eliminate the primary interferer. The "perfect IC" can also be considered as the situation that $\Delta=\infty$

In Table $4.2, \Delta$ ranges from 0 to $30 \mathrm{~dB}$. Since the MS always selects the sector with the highest received power as the serving sector, $\Delta$ will not be less than $0 \mathrm{~dB}$. 


\begin{tabular}{|c|c|c|c|c|c|c|c|c|c|}
\hline$\Delta(\mathrm{dB}) \backslash \mathrm{SNR}(\mathrm{dB})$ & -5 & -4 & -3 & -2 & -1 & 0 & 1 & 2 & 3 \\
\hline 0 & 0.000 & 0.000 & 0.001 & 0.002 & 0.006 & 0.015 & 0.033 & 0.066 & 0.122 \\
\hline 3 & 0.000 & 0.000 & 0.001 & 0.004 & 0.010 & 0.022 & 0.045 & 0.082 & 0.134 \\
\hline 6 & 0.000 & 0.000 & 0.002 & 0.005 & 0.014 & 0.033 & 0.063 & 0.109 & 0.170 \\
\hline 9 & 0.000 & 0.001 & 0.002 & 0.007 & 0.019 & 0.042 & 0.081 & 0.137 & 0.210 \\
\hline 12 & 0.000 & 0.001 & 0.002 & 0.008 & 0.022 & 0.049 & 0.094 & 0.159 & 0.243 \\
\hline 15 & 0.000 & 0.001 & 0.003 & 0.009 & 0.024 & 0.053 & 0.102 & 0.172 & 0.264 \\
\hline 18 & 0.000 & 0.001 & 0.003 & 0.009 & 0.025 & 0.055 & 0.107 & 0.181 & 0.276 \\
\hline 21 & 0.000 & 0.001 & 0.003 & 0.010 & 0.025 & 0.057 & 0.110 & 0.185 & 0.282 \\
\hline 24 & 0.000 & 0.001 & 0.003 & 0.010 & 0.026 & 0.057 & 0.111 & 0.187 & 0.286 \\
\hline 27 & 0.000 & 0.001 & 0.003 & 0.010 & 0.026 & 0.058 & 0.111 & 0.188 & 0.288 \\
\hline 30 & 0.000 & 0.001 & 0.003 & 0.010 & 0.026 & 0.058 & 0.112 & 0.189 & 0.289 \\
\hline perfect IC & 0.000 & 0.001 & 0.003 & 0.010 & 0.026 & 0.058 & 0.113 & 0.190 & 0.290 \\
\hline$\Delta(\mathrm{dB}) \backslash \mathrm{SNR}(\mathrm{dB})$ & 4 & 5 & 6 & 7 & 8 & 9 & 10 & 11 & 12 \\
\hline 0 & 0.205 & 0.316 & 0.444 & 0.576 & 0.701 & 0.803 & 0.879 & 0.929 & 0.961 \\
\hline 3 & 0.206 & 0.298 & 0.405 & 0.524 & 0.642 & 0.750 & 0.838 & 0.902 & 0.944 \\
\hline 6 & 0.246 & 0.334 & 0.431 & 0.532 & 0.635 & 0.728 & 0.810 & 0.876 & 0.924 \\
\hline 9 & 0.296 & 0.390 & 0.487 & 0.583 & 0.671 & 0.750 & 0.818 & 0.873 & 0.917 \\
\hline 12 & 0.339 & 0.441 & 0.543 & 0.638 & 0.720 & 0.789 & 0.847 & 0.891 & 0.926 \\
\hline 15 & 0.368 & 0.477 & 0.584 & 0.680 & 0.760 & 0.825 & 0.876 & 0.914 & 0.941 \\
\hline 18 & 0.384 & 0.498 & 0.608 & 0.705 & 0.785 & 0.849 & 0.896 & 0.931 & 0.954 \\
\hline 21 & 0.393 & 0.509 & 0.621 & 0.718 & 0.799 & 0.863 & 0.908 & 0.941 & 0.962 \\
\hline 24 & 0.398 & 0.515 & 0.628 & 0.726 & 0.806 & 0.869 & 0.915 & 0.946 & 0.967 \\
\hline 27 & 0.401 & 0.518 & 0.631 & 0.730 & 0.811 & 0.873 & 0.918 & 0.949 & 0.969 \\
\hline 30 & 0.402 & 0.520 & 0.633 & 0.732 & 0.812 & 0.874 & 0.919 & 0.950 & 0.970 \\
\hline perfect IC & 0.402 & 0.521 & 0.634 & 0.732 & 0.814 & 0.876 & 0.921 & 0.951 & 0.970 \\
\hline$\Delta(\mathrm{dB}) \backslash \mathrm{SNR}(\mathrm{dB})$ & 13 & 14 & 15 & 16 & 17 & 18 & 19 & 20 & 21 \\
\hline 0 & 0.978 & 0.989 & 0.994 & 0.997 & 0.998 & 0.999 & 1.000 & 1.000 & 1.000 \\
\hline 3 & 0.970 & 0.984 & 0.992 & 0.996 & 0.998 & 0.999 & 1.000 & 1.000 & 1.000 \\
\hline 6 & 0.956 & 0.976 & 0.988 & 0.994 & 0.997 & 0.999 & 0.999 & 1.000 & 1.000 \\
\hline 9 & 0.948 & 0.970 & 0.984 & 0.991 & 0.996 & 0.998 & 0.999 & 1.000 & 1.000 \\
\hline 12 & 0.952 & 0.969 & 0.982 & 0.990 & 0.995 & 0.997 & 0.999 & 0.999 & 1.000 \\
\hline 15 & 0.961 & 0.975 & 0.984 & 0.991 & 0.995 & 0.997 & 0.998 & 0.999 & 1.000 \\
\hline 18 & 0.970 & 0.981 & 0.988 & 0.993 & 0.995 & 0.997 & 0.998 & 0.999 & 1.000 \\
\hline 21 & 0.976 & 0.985 & 0.991 & 0.995 & 0.997 & 0.998 & 0.999 & 0.999 & 1.000 \\
\hline 24 & 0.980 & 0.988 & 0.993 & 0.996 & 0.998 & 0.999 & 0.999 & 1.000 & 1.000 \\
\hline 27 & 0.981 & 0.989 & 0.994 & 0.997 & 0.998 & 0.999 & 0.999 & 1.000 & 1.000 \\
\hline 30 & 0.982 & 0.990 & 0.994 & 0.997 & 0.998 & 0.999 & 1.000 & 1.000 & 1.000 \\
\hline perfect IC & 0.983 & 0.991 & 0.995 & 0.997 & 0.998 & 0.999 & 1.000 & 1.000 & 1.000 \\
\hline
\end{tabular}

Table 4.2: Connection rate on SUI-5 channel with $N_{S}=2, N_{A}=1$. 


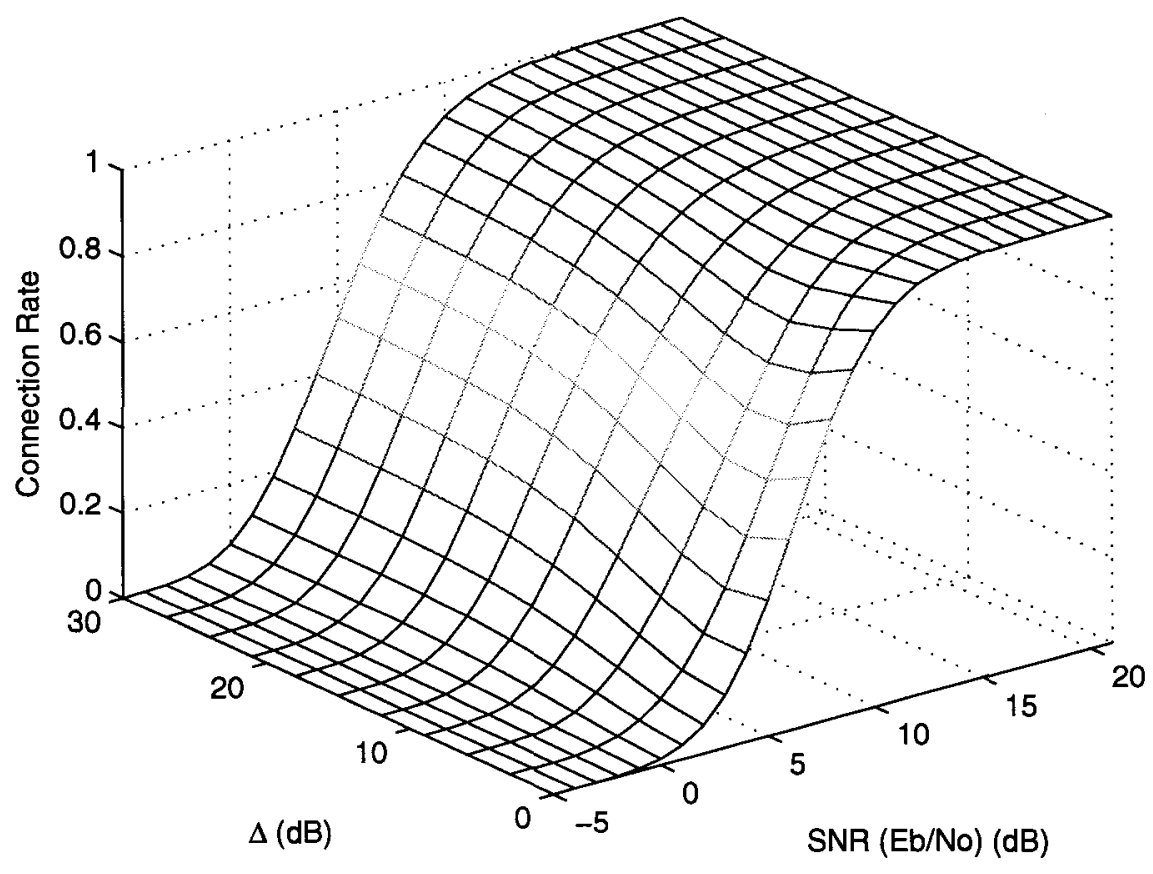

Figure 4.3: 3-D connection rate graph on SUI-5 channel with $N_{S}=2, N_{A}=1$.

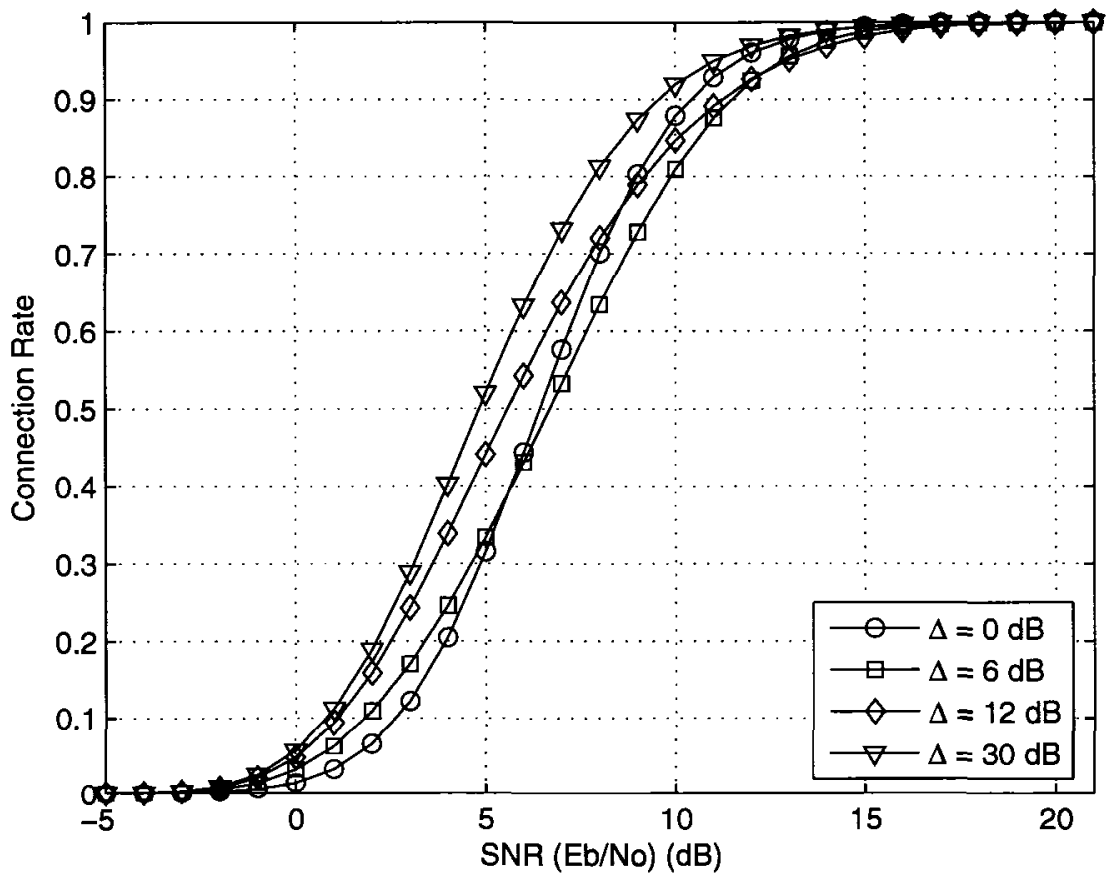

Figure 4.4: Connection rate on SUI-5 channel with $N_{S}=2, N_{A}=1$. $\Delta=0,6,12$ and $30 \mathrm{~dB}$. 
Noticing that the discrepancy between the $30 \mathrm{~dB}$ row and the "perfect IC" row is very small, when $\Delta>30 \mathrm{~dB}$ we treat it as $30 \mathrm{~dB}$. The SNR in this table ranges from $-5 \mathrm{~dB}$ to $21 \mathrm{~dB}$. When $\mathrm{SNR}<-5 \mathrm{~dB}$, we assume the connection rate is always 0 ; when $\mathrm{SNR}>21 \mathrm{~dB}$, we assume the connection rate is 1 . Two-dimension linear interpolation is used to calculate the correction rate for those values of SNR and $\Delta$ that can not be found in this table.

Depending on a user's position in the cell, it will experience different path losses and shadowing to the surrounding transmitters. As such, the SNR and $\Delta$ are random variables, so the connection rate is also a random variable. In this section, we study the average connection rate of a user, and the cumulative density function of the connection rate. These are determined by randomly placing the user uniformly within sector 0 of cell 0 five million times. With each placement the antenna gains and path losses to each transmit antenna are calculated, and a random lognormal shadowing value is selected for the signals from each base station. When cell sectoring is used, the signal for each sector of a cell suffers from the same path loss and shadowing, but different antenna gains.

Figures $4.5-4.16$ show the simulation results. For any figure, Part (a) shows the frequency group layout, Part (b) shows some statistics of the results, and Part (c) shows the cumulative distribution function (CDF) of the connection rate. In Part (b), the average connection rate is the average connection rate of all users, the median connection rate is the connection rate when $\mathrm{CDF}=0.5$, the $10 \%$ outage rate is the maximum connection rate of those $10 \%$ of users with the lowest connection rate, and the 0.2 outage probability is the fraction of users whose connection rate is less than 
0.2 bits per channel use.

Figure 4.5 is for the case when all the cells use the same frequency group (group A), which means that the cell frequency reuse factor is 1 . No sectoring in this case means the sectors / cell ratio is 1 . And naturally, the sector frequency reuse factor is 1 . We denote this case as Scheme 111. From Part (b) we can see that the average connection rate of the "IC" is obviously higher than that of the "no IC", but lower than that of the "perfect IC". This means that the IC can effectively reduce the primary CCI but that residual interference still exists. The median connection rate difference among these three cases is even bigger. If no IC is used, $50 \%$ of the users have a connection rate greater than 0.33 ; if $\mathrm{IC}$ is applied, the median connection rate rises to 0.53 ; if the primary interferer could be thoroughly eliminated, the median connection rate would improve to 0.66 . From the two rates above, we might conclude that the performace of Scheme 111 is good. However, the $10 \%$ outage rate tells us that at least $10 \%$ of the users have practically no service $(0.004,0.015$ and 0.031 for "no IC", "IC" and "perfect IC" respectively), which is unreasonable for a practical network. The 0.2 outage probability statistic also shows that many users suffer from low connection rate, resulting in frequent re-transmission. From all four parameters above, we can see that the IC can effectively improve the performance. The figure of Part (c) also shows this improvement.

Increasing the number of sectors per cell to 3 , while maintaining a cell frequency reuse factor of 1 and a sector reuse factor of 1 give us Scheme 131, which is shown in Figure 4.6. Though each cell is split into 3 sectors, all sectors still use the same frequency group. Thus the number of CCI sources increases by a factor of 3 . For 


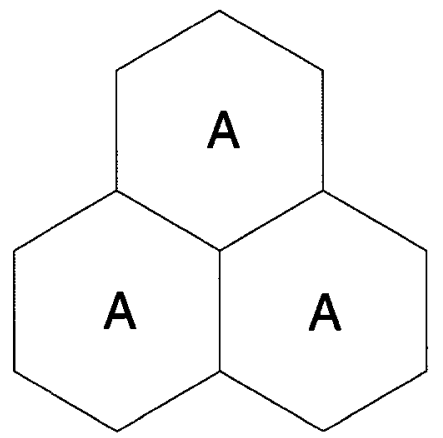

(a) Layout.

\begin{tabular}{|l|c|c|c|}
\hline & no IC & IC & $\begin{array}{c}\text { perfect } \\
\text { IC }\end{array}$ \\
\hline \hline Ave. connection rate & 0.446 & 0.527 & 0.586 \\
\hline Median connection rate & 0.329 & 0.524 & 0.659 \\
\hline $10 \%$ outage rate & 0.004 & 0.015 & 0.031 \\
\hline 0.2 outage probability & 0.423 & 0.315 & 0.250 \\
\hline
\end{tabular}

(b) Statistics.

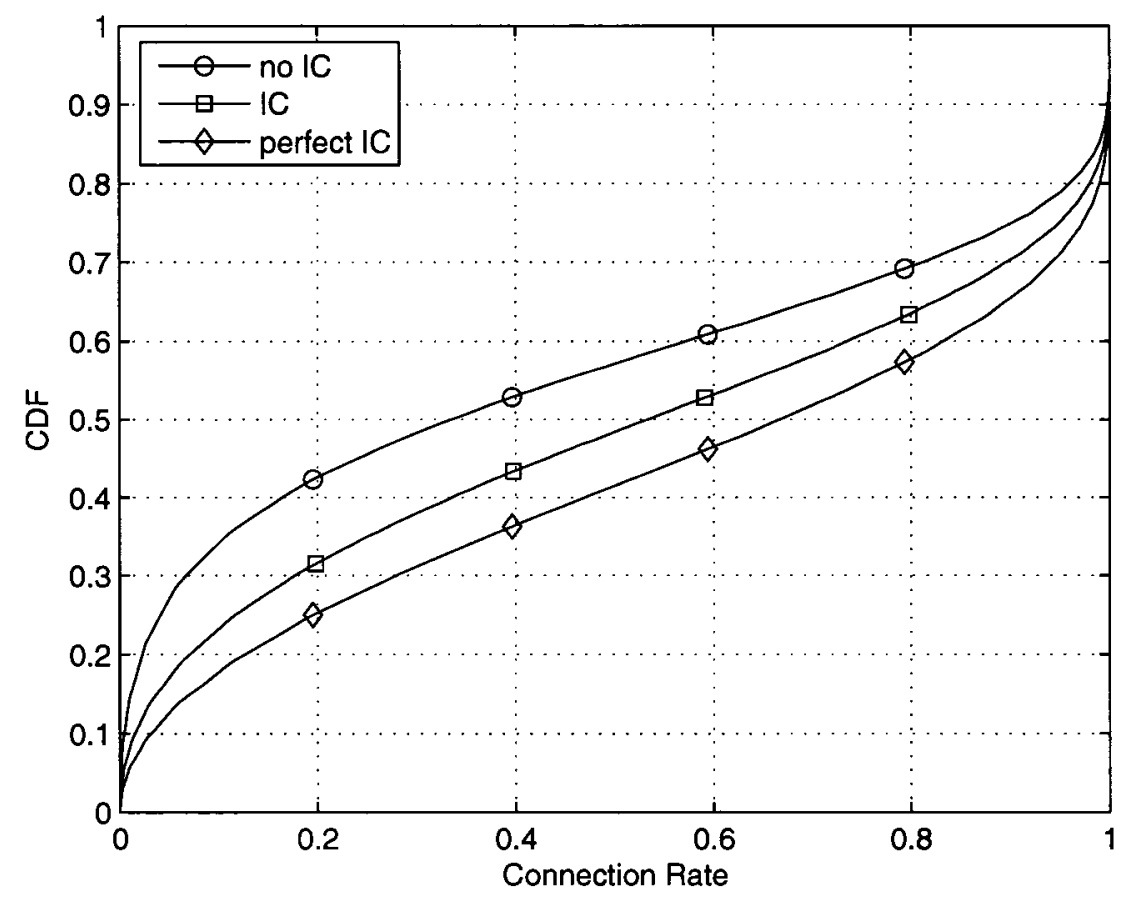

(c) $\mathrm{CDF}$ of connection rate.

Figure 4.5: Scheme 111 (cell reuse factor $=1$, sectors $/$ cell $=1$, sector reuse factor $=1$ ). 


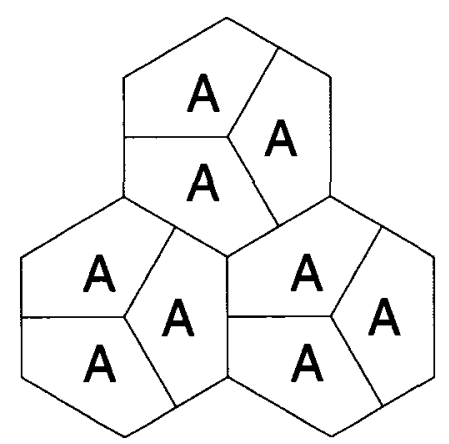

(a) Layout.

\begin{tabular}{|l|c|c|c|}
\hline & no IC & IC & $\begin{array}{c}\text { Perfect } \\
\text { IC }\end{array}$ \\
\hline \hline Ave. connection rate & 0.363 & 0.443 & 0.499 \\
\hline Median connection rate & 0.187 & 0.357 & 0.479 \\
\hline $10 \%$ outage rate & 0.002 & 0.006 & 0.012 \\
\hline 0.2 outage probability & 0.501 & 0.397 & 0.331 \\
\hline
\end{tabular}

(b) Statistics.

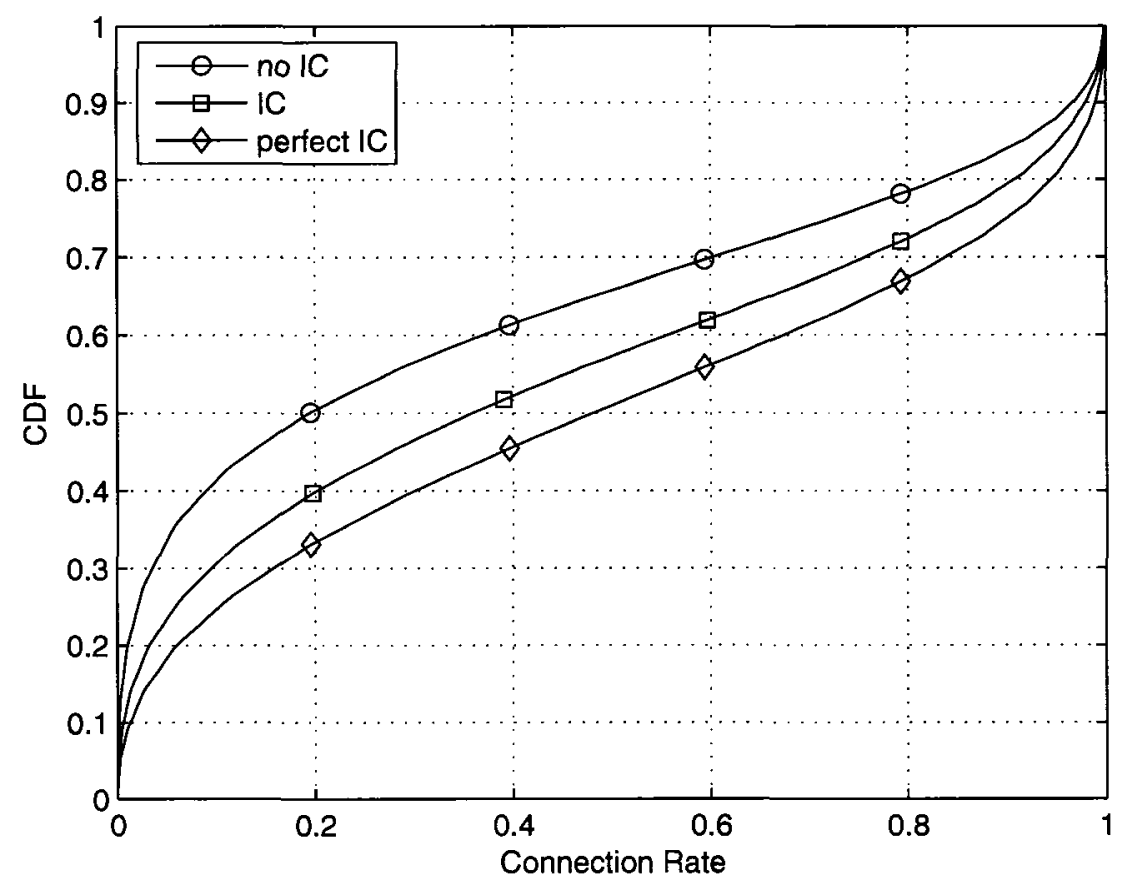

(c) CDF of connection rate.

Figure 4.6: Scheme 131 (cell reuse factor $=1$, sectors $/$ cell $=3$, sector reuse factor $=1$ ). 
the "no IC" case, the median connection rate is smaller than 0.19 ; for the "IC" case, the rate is 0.36 ; for the "perfect IC", the number is 0.48 . Although the IC is able to reduce the CCI and improve the performance, overall this more aggressive frequency reuse scheme, with its associated increase in CCI, leads to worse performance than Scheme 111.

An even more aggressive frequency reuse scheme occurs when the number of sectors per cell increases to 6 (Scheme 161). As shown in Figure 4.7, the additional CCI further degrades performance, and the median connection rate of the "no IC", the "IC" and the "perfect IC" cases are less than $0.13,0.25$ and 0.35 respectively, worse than those of scheme 131 .

To prevent high levels of CCI in the first place, less aggressive frequency reuse schemes can be deployed, such as Scheme 133 shown in Figure 4.8. This is a 3sector application, but different frequency groups (groups A, B, and C) are used for each sector within the cell. As expected, we obtain a significant improvement in the connection rate, and the connection rate for most users is above 0.9 , for all three cases.

Other moderate schemes are applicable for the 6-sector layout, such as Scheme 163 and Scheme 166, as shown in Figures 4.9 and 4.10. Compared to Scheme 161, we can see a great improvement in connection rates.

For the schemes described above, all cells use the same frequency groups. In practice, different frequency groups are allocated to neighboring cells in order to avoid strong CCI along the cell borders. In the following we consider a cell frequency reuse factor of 3 . 


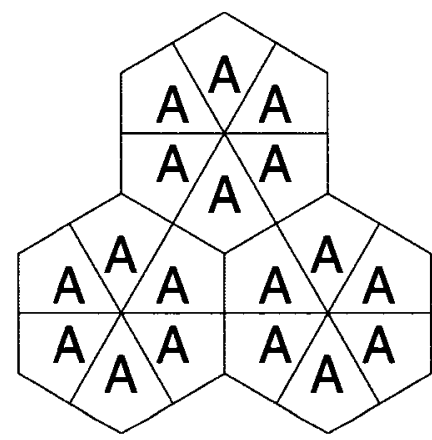

(a) Layout.

\begin{tabular}{|l|c|c|c|}
\hline & no IC & IC & $\begin{array}{c}\text { perfect } \\
\text { IC }\end{array}$ \\
\hline \hline Ave. connection rate & 0.311 & 0.371 & 0.424 \\
\hline Median connection rate & 0.125 & 0.249 & 0.352 \\
\hline $10 \%$ outage rate & 0.001 & 0.002 & 0.005 \\
\hline 0.2 outage probability & 0.555 & 0.456 & 0.393 \\
\hline
\end{tabular}

(b) Statistics.

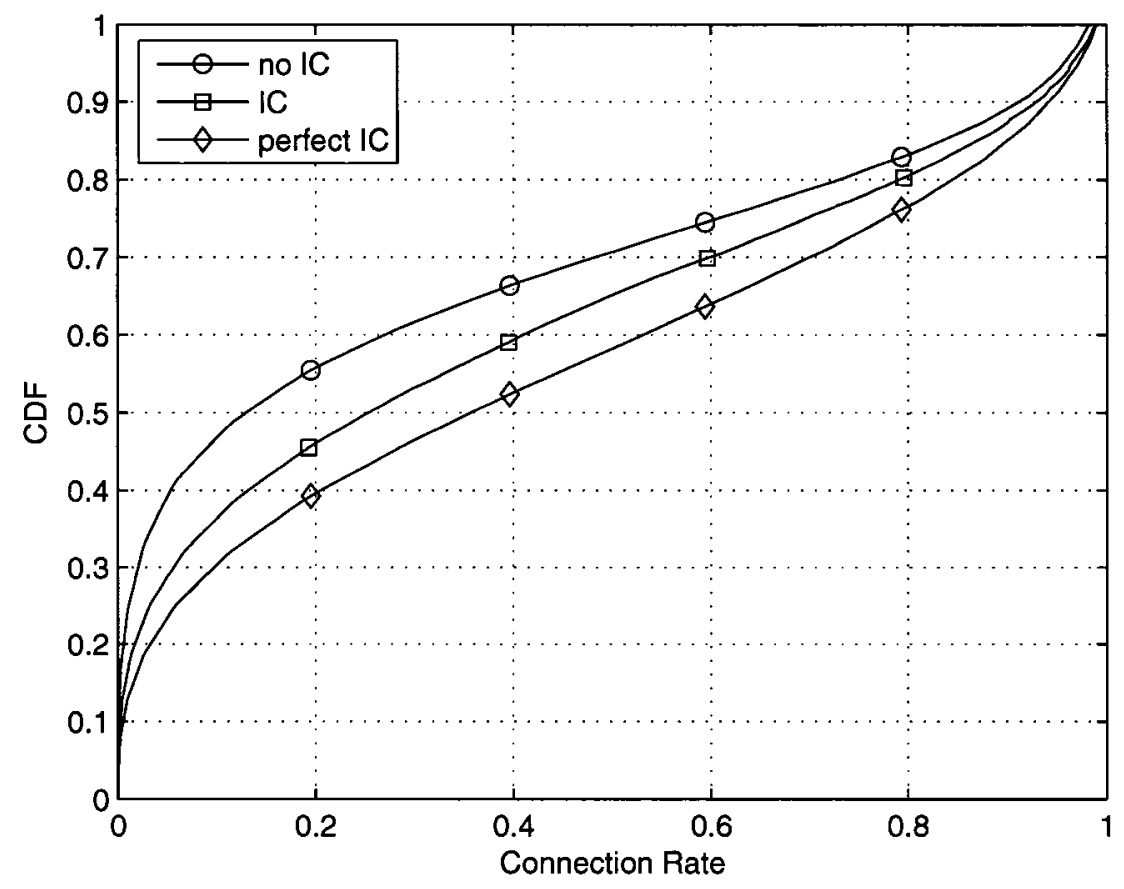

(c) CDF of connection rate.

Figure 4.7: Scheme 161 (cell reuse factor $=1$, sectors $/$ cell=6, sector reuse factor $=1$ ). 


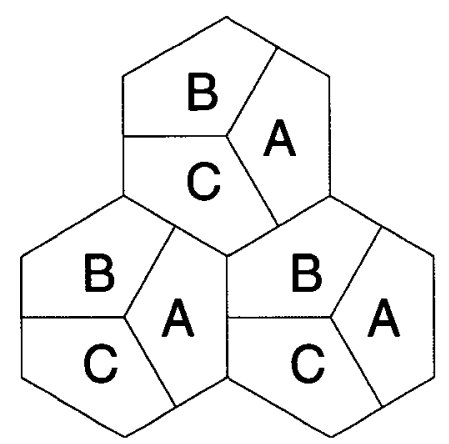

(a) Layout.

\begin{tabular}{|l|c|c|c|}
\hline & no IC & IC & $\begin{array}{c}\text { perfect } \\
\text { IC }\end{array}$ \\
\hline \hline Ave. connection rate & 0.821 & 0.880 & 0.914 \\
\hline Median connection rate & 0.952 & 0.976 & 0.988 \\
\hline $10 \%$ outage rate & 0.374 & 0.585 & 0.708 \\
\hline 0.2 outage probability & 0.045 & 0.015 & 0.007 \\
\hline
\end{tabular}

(b) Statistics.

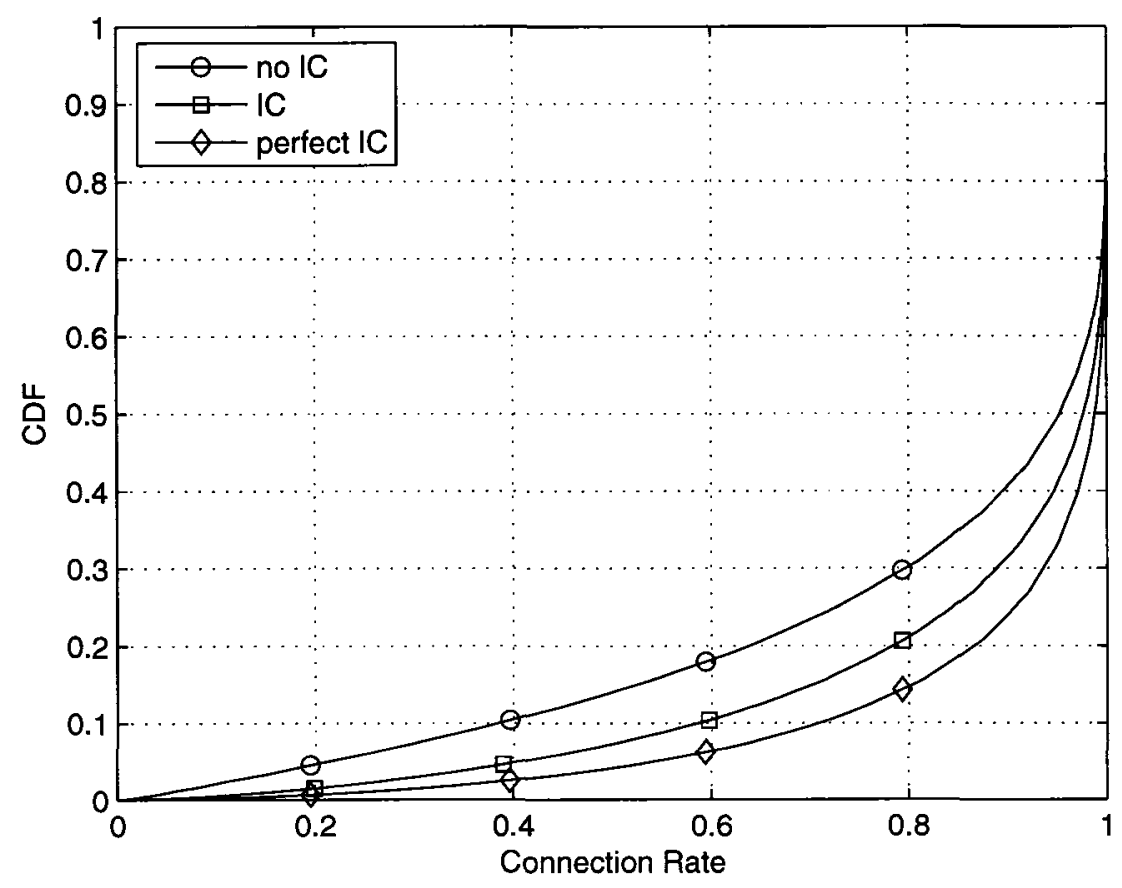

(c) CDF of connection rate.

Figure 4.8: Scheme 133 (cell reuse factor $=1$, sectors $/$ cell=3, sector reuse factor $=3$ ). 


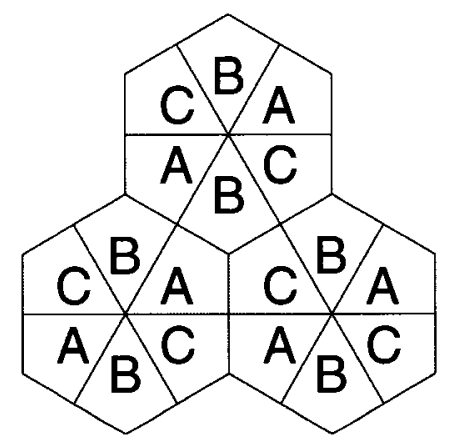

(a) Layout.

\begin{tabular}{|l|c|c|c|}
\hline & no IC & IC & $\begin{array}{c}\text { perfect } \\
\text { IC }\end{array}$ \\
\hline \hline Ave. connection rate & 0.771 & 0.832 & 0.873 \\
\hline Median connection rate & 0.905 & 0.943 & 0.966 \\
\hline $10 \%$ outage rate & 0.275 & 0.447 & 0.572 \\
\hline 0.2 outage probability & 0.071 & 0.029 & 0.015 \\
\hline
\end{tabular}

(b) Statistics.

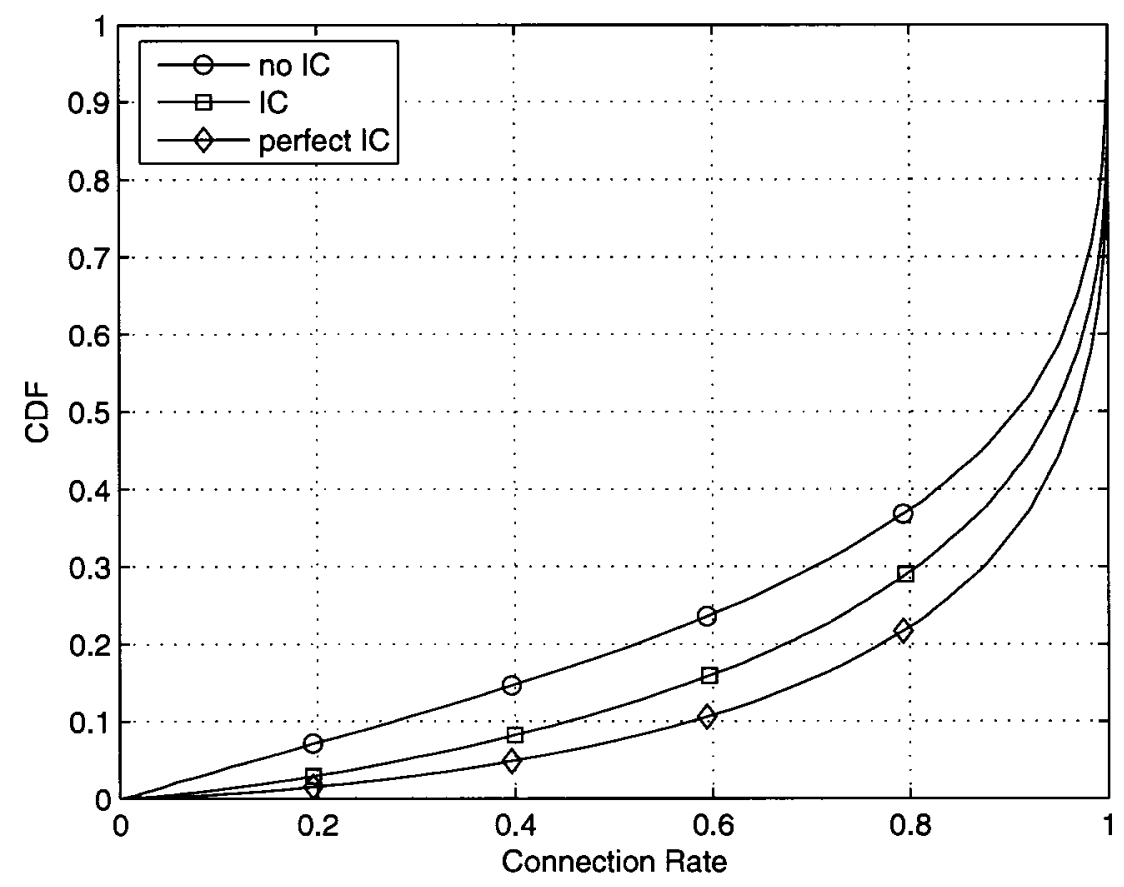

(c) CDF of connection rate.

Figure 4.9: Scheme 163 (cell reuse factor $=1$, sectors / cell=6, sector reuse factor $=3$ ). 


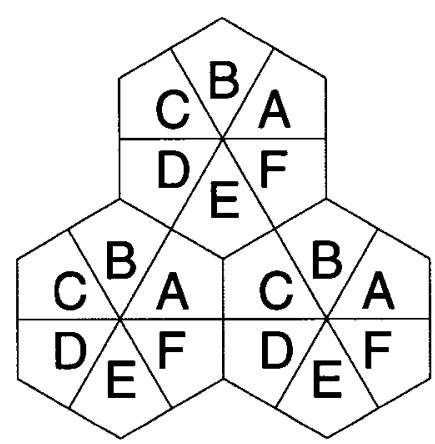

(a) Layout.

\begin{tabular}{|l|c|c|c|}
\hline & no IC & IC & $\begin{array}{c}\text { IC } \\
\text { ICfect }\end{array}$ \\
\hline \hline Ave. connection rate & 0.935 & 0.961 & 0.975 \\
\hline Median connection rate & 0.992 & 0.996 & 0.998 \\
\hline $10 \%$ outage rate & 0.785 & 0.876 & 0.925 \\
\hline 0.2 outage probability & 0.003 & 0.000 & 0.000 \\
\hline
\end{tabular}

(b) Statistics.

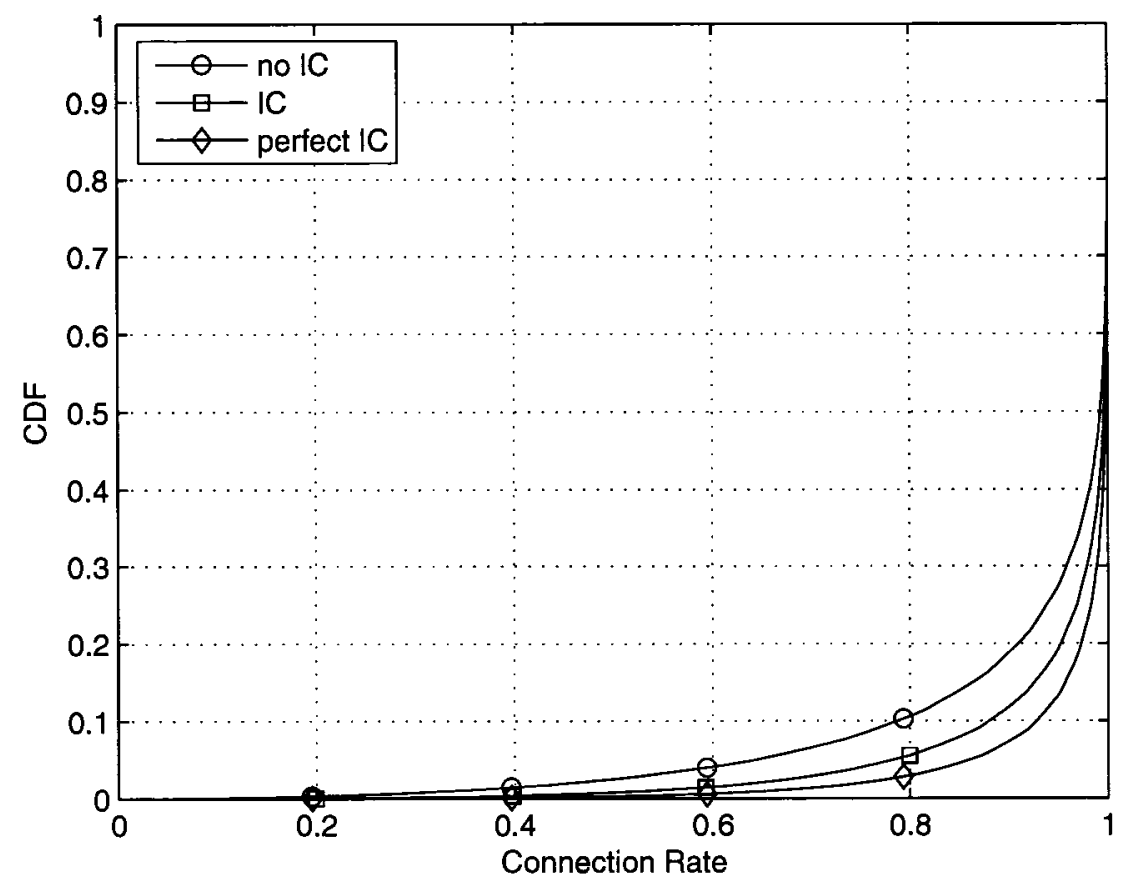

(c) CDF of connection rate.

Figure 4.10: Scheme 166 (cell reuse factor $=1$, sectors $/$ cell $=6$, sector reuse factor $=6$ ). 


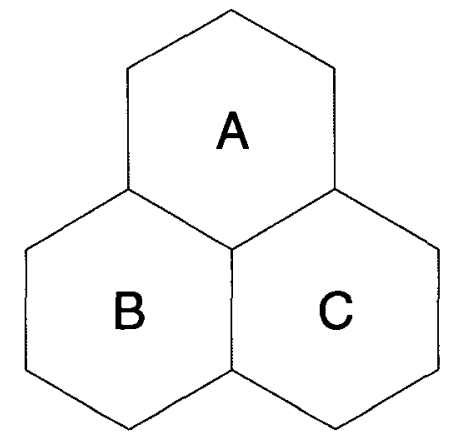

(a) Layout.

\begin{tabular}{|l|c|c|c|}
\hline & no IC & IC & \begin{tabular}{c} 
IC \\
\hline
\end{tabular} \\
\hline \hline Ave. connection rate & 0.881 & 0.929 & 0.953 \\
\hline Median connection rate & 0.985 & 0.994 & 0.997 \\
\hline $10 \%$ outage rate & 0.560 & 0.750 & 0.848 \\
\hline 0.2 outage probability & 0.022 & 0.005 & 0.002 \\
\hline
\end{tabular}

(b) Statistics.

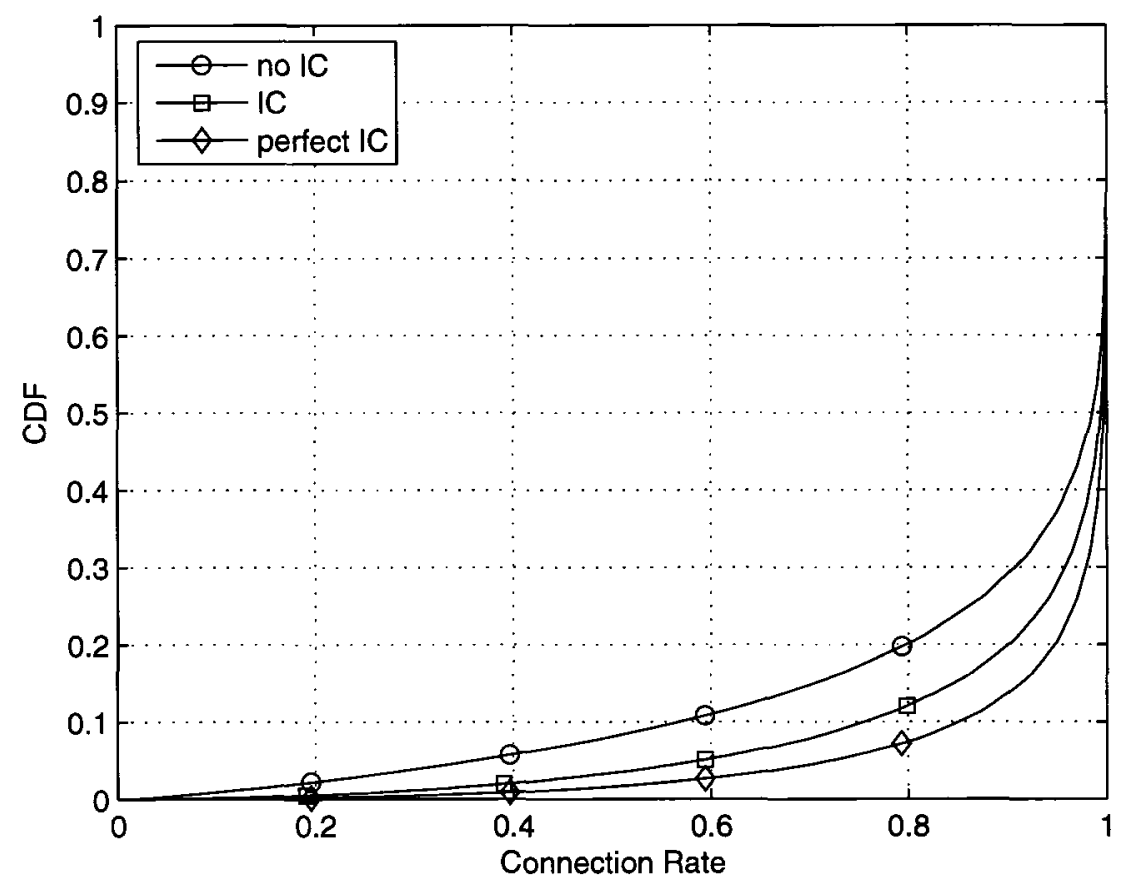

(c) $\mathrm{CDF}$ of connection rate.

Figure 4.11: Scheme 311 (cell reuse factor $=3$, sectors $/$ cell=1, sector reuse factor $=1$ ). 
Scheme 311 (Figure 4.11) has three different frequency groups among three neighboring cells. This is comparable to Scheme 133 (Figure 4.8) in which there are also three different frequency groups. The connection rate curves of both schemes are also similar, but the performance of Scheme 311 is slightly better. This difference is due to the fact that, even though directional antennas are used in the sectorized scheme, the interfering transmitters are closer to the user than in Scheme 311, so the CCI is stronger. We may conclude that, from the point of view of connection rate, the cell frequency reuse is slightly better than sector frequency reuse.

Scheme 331 (Figure 4.12) is also comparable with Scheme 133 (Figure 4.8). Both schemes have the same frequency groups and sector structure, but the frequency group distribution patterns are different. Clearly, the scheme with more reasonable pattern (Scheme 133) give better performance. Compared to Scheme 133, there is strong CCI along the sector borders of Scheme 331, which adversely affects the performance.

Scheme 333 (Figure 4.13) is a very reliable scheme. By using nine different frequency groups spread over three cells, strong CCI rarely occurs, and almost all the users have a connection rate over 0.8 .

The comparison between Scheme 361 (Figure 4.14) and Scheme 163 (Figure 4.9) is similar to that between Scheme 331 (Figure 4.12) and Scheme 133 (Figure 4.8). We may conclude that an evenly distributed frequency distributed pattern is important to cellular network design. We also notice the slight difference of improvement, which the "IC" and the "perfect IC" can provide over the "no IC" case, between these two schemes. Take the average connection rate for example. The statistics for Scheme 163 are $0.77,0.83$, and 0.87 for "no IC", "IC", and "perfect IC" respectively; for Scheme 


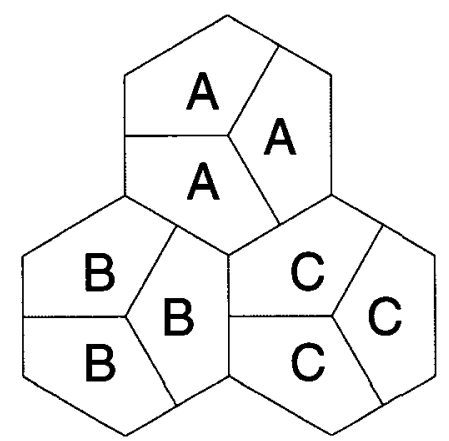

(a) Layout.

\begin{tabular}{|l|c|c|c|}
\hline & no IC & IC & $\begin{array}{c}\text { perfect } \\
\text { IC }\end{array}$ \\
\hline \hline Ave. connection rate & 0.756 & 0.851 & 0.897 \\
\hline Median connection rate & 0.873 & 0.938 & 0.968 \\
\hline $10 \%$ outage rate & 0.275 & 0.532 & 0.668 \\
\hline 0.2 outage probability & 0.065 & 0.016 & 0.007 \\
\hline
\end{tabular}

(b) Statistics.

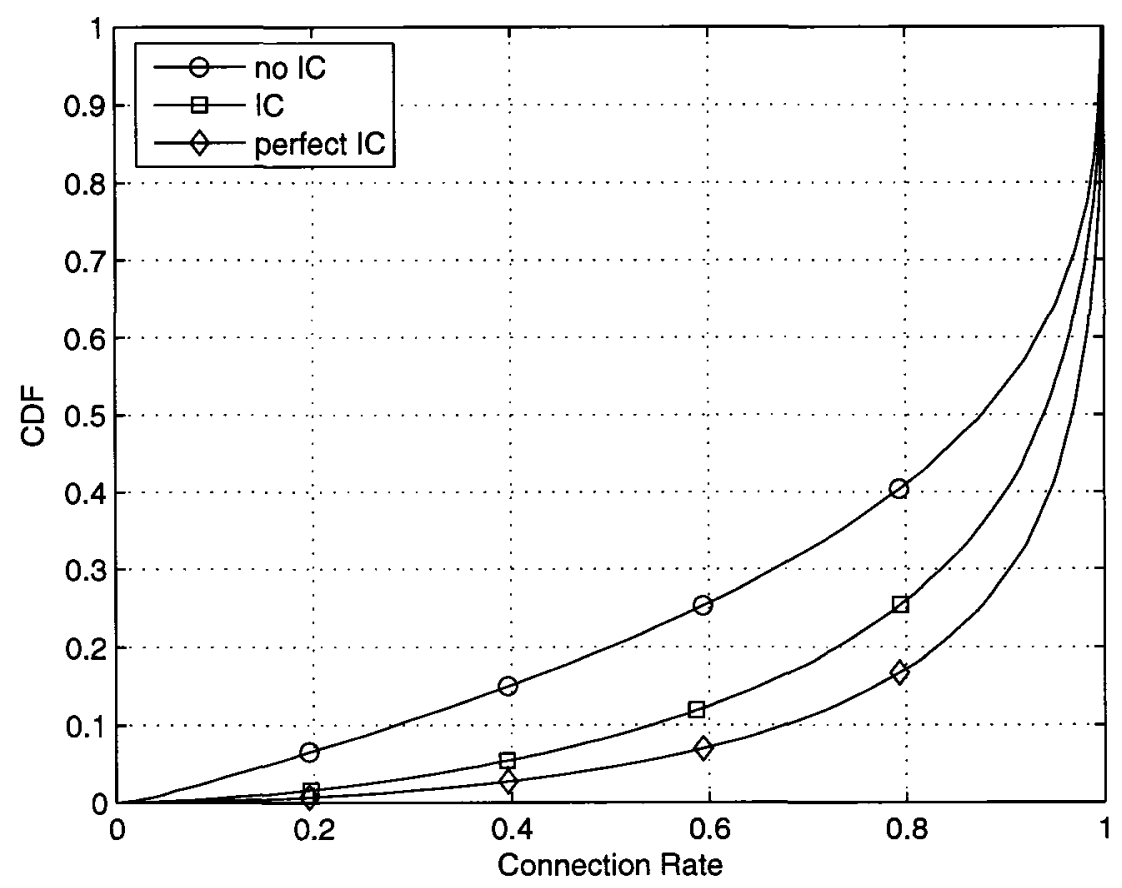

(c) CDF of connection rate.

Figure 4.12: Scheme 331 (cell reuse factor $=3$, sectors $/$ cell=3, sector reuse factor $=1$ ). 


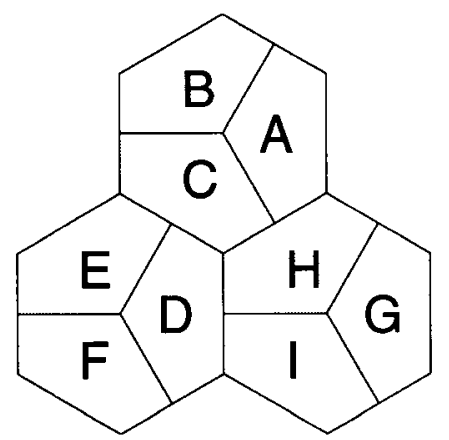

(a) Layout.

\begin{tabular}{|l|c|c|c|}
\hline & no IC & IC & $\begin{array}{c}\text { perfect } \\
\text { IC }\end{array}$ \\
\hline \hline Ave. connection rate & 0.986 & 0.993 & 0.996 \\
\hline Median connection rate & 1.000 & 1.000 & 1.000 \\
\hline $10 \%$ outage rate & 0.964 & 0.983 & 0.991 \\
\hline 0.2 outage probability & 0.000 & 0.000 & 0.000 \\
\hline
\end{tabular}

(b) Statistics.

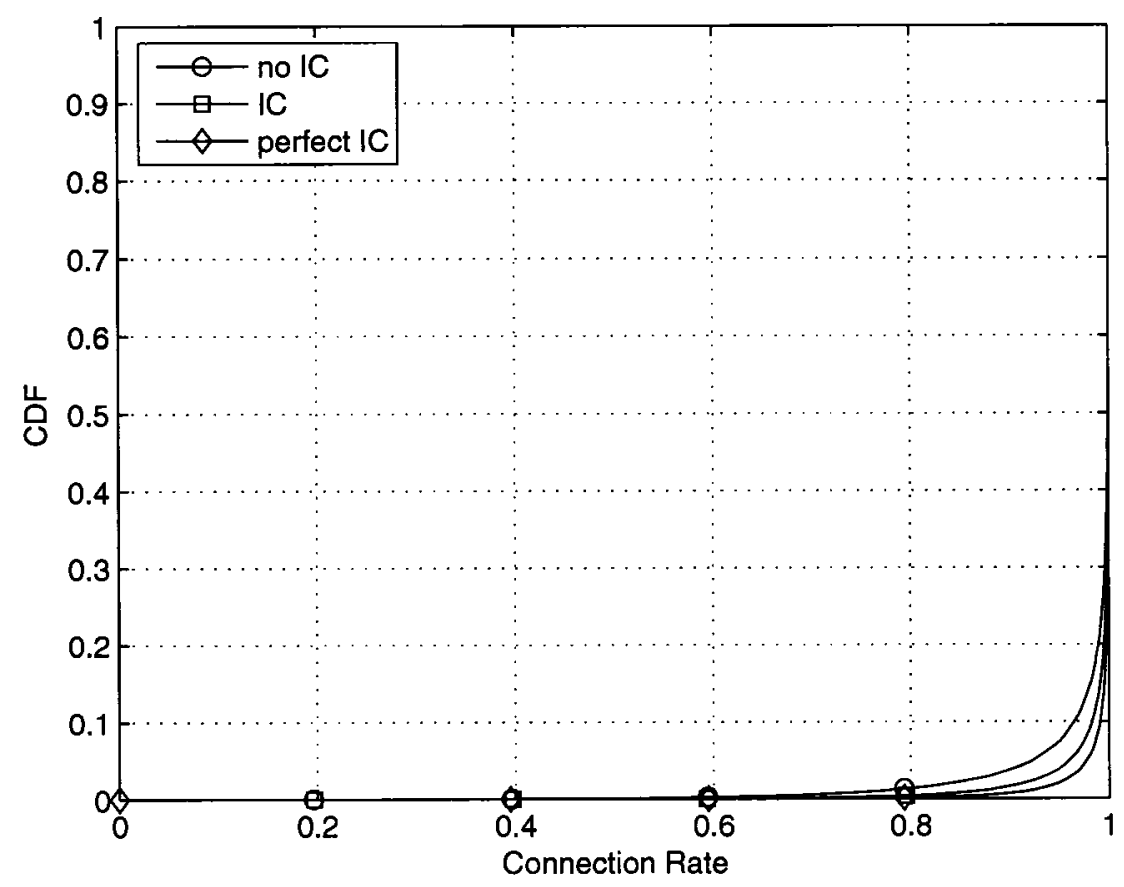

(c) CDF of connection rate.

Figure 4.13: Scheme 333 (cell reuse factor $=3$, sectors $/$ cell $=3$, sector reuse factor $=3$ ). 


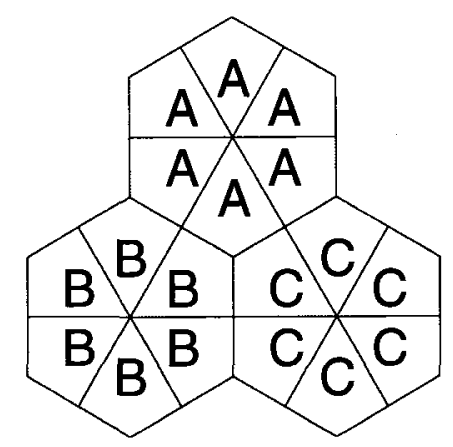

(a) Layout.

\begin{tabular}{|l|c|c|c|}
\hline & no IC & IC & $\begin{array}{c}\text { perfect } \\
\text { IC }\end{array}$ \\
\hline Ave. connection rate & 0.653 & 0.733 & 0.797 \\
\hline Median connection rate & 0.745 & 0.813 & 0.873 \\
\hline $10 \%$ outage rate & 0.148 & 0.339 & 0.479 \\
\hline 0.2 outage probability & 0.126 & 0.043 & 0.021 \\
\hline
\end{tabular}

(b) Statistics.

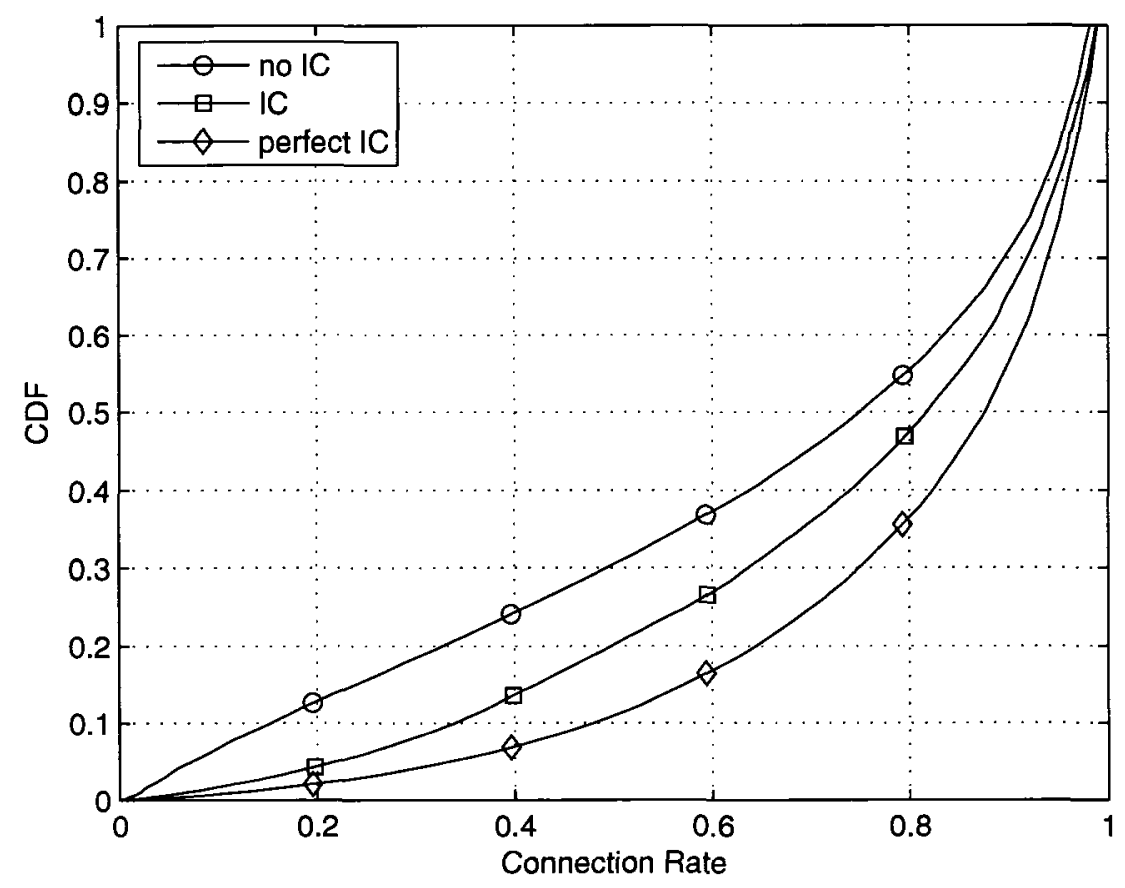

(c) CDF of connection rate.

Figure 4.14: Scheme 361 (cell reuse factor $=3$, sectors $/$ cell $=6$, sector reuse factor $=1$ ). 
361 , these numbers become $0.65,0.73$, and 0.80 . Clearly, the IC (and perfect IC) of Scheme 361 is able to provide a slightly better improvement over "no IC" than that of Scheme 163 can do. This is because the IC is more effective at canceling a strong CCI than at canceling a weak one.

The comparison between Scheme 363 (Figure 4.15) and Scheme 166 (Figure 4.10) is interesting. In Scheme 363, there are nine frequency groups, more than Scheme 166's six groups. Thus we might expect Scheme 363 to give better performance. However, for the no IC case, Scheme 363 actually performs worse. The reason is that the primary interferer in Scheme 363 is so dominant that it significantly degrades the connection rate performance. Once the primary interferer is (partially) eliminated by the IC, the connection rate improves sufficiently. When the IC is not applied, the average connection rates are 0.922 for Scheme 363 and 0.935 for Scheme 166; when the IC is applied, the rates are 0.970 for Scheme 363 and 0.961 for Scheme 166. The similar pattern can also be found with the other statistics.

Scheme 366 (Figure 4.16), with 18 frequency groups, suffers the least CCI among all the schemes we considered. There is much less strong CCI left for interference canceling. Thus the improvement of the IC is very limited.

From all twelve schemes above, we can see that the IC can effectively improve the system performance in various statistic parameters. However, the residual interference prevents the performance from further improvement (to the "perfect IC" level). We can also see that the IC is more effective at canceling a strong primary CCI than a weak one, because a weak primary CCI is possibly unable to be detected by the receiver and is treated as noise. Another important point is that though the IC is 


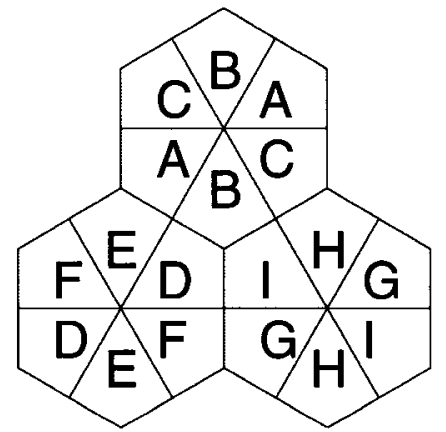

(a) Layout.

\begin{tabular}{|l|c|c|c|}
\hline & no IC & IC & $\begin{array}{c}\text { perfect } \\
\text { IC }\end{array}$ \\
\hline \hline Ave. connection rate & 0.922 & 0.970 & 0.985 \\
\hline Median connection rate & 0.983 & 0.995 & 0.998 \\
\hline $10 \%$ outage rate & 0.745 & 0.909 & 0.958 \\
\hline 0.2 outage probability & 0.003 & 0.000 & 0.000 \\
\hline
\end{tabular}

(b) Statistics.

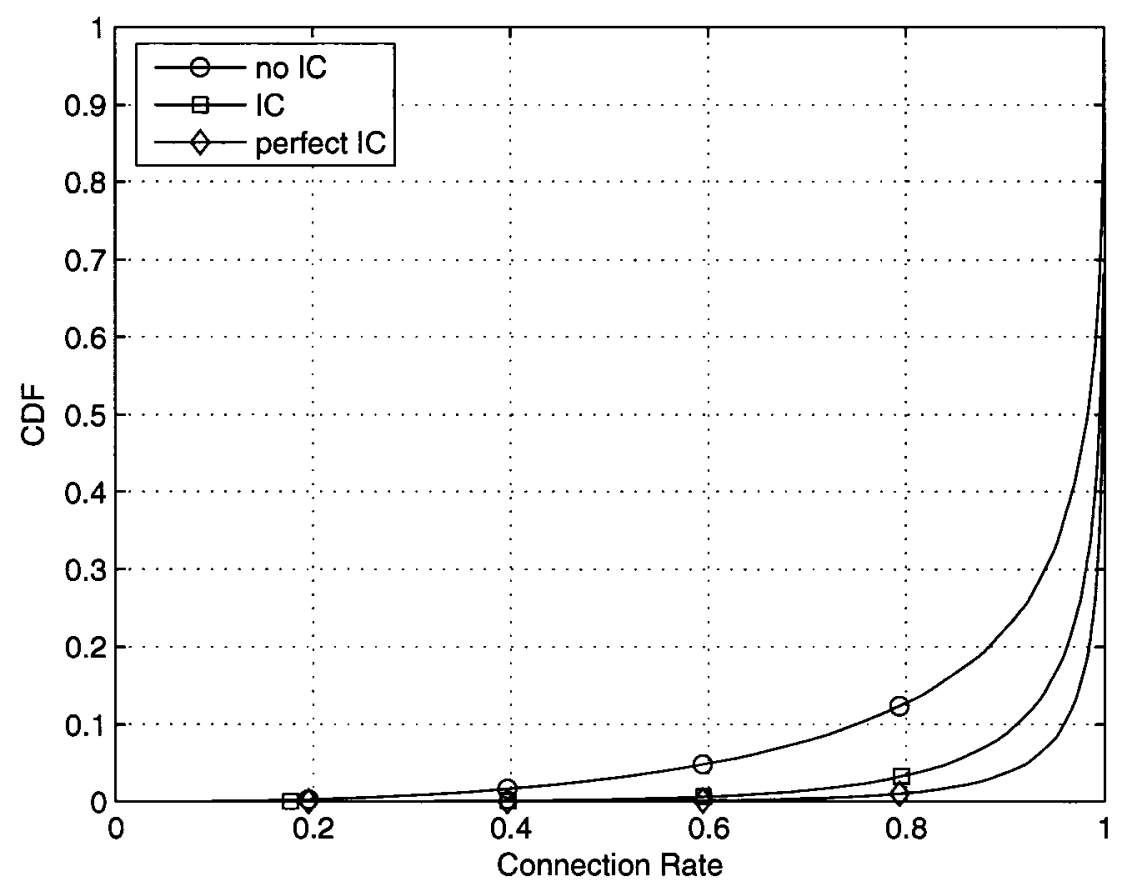

(c) CDF of connection rate.

Figure 4.15: Scheme 363 (cell reuse factor $=3$, sectors $/$ cell $=6$, sector reuse factor $=3$ ). 


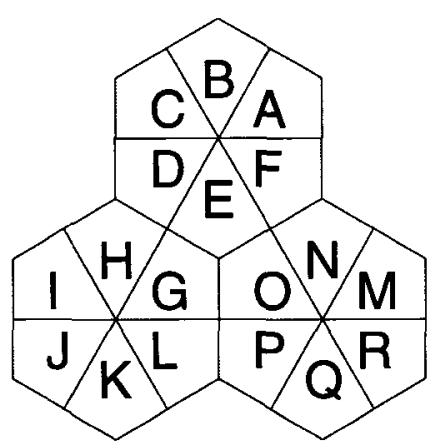

(a) Layout.

\begin{tabular}{|l|c|c|c|}
\hline & no IC & IC & $\begin{array}{c}\text { perfect } \\
\text { IC }\end{array}$ \\
\hline \hline Ave. connection rate & 0.994 & 0.997 & 0.998 \\
\hline Median connection rate & 1.000 & 1.000 & 1.000 \\
\hline $10 \%$ outage rate & 0.987 & 0.994 & 0.996 \\
\hline 0.2 outage probability & 0.000 & 0.000 & 0.000 \\
\hline
\end{tabular}

(b) Statistics.

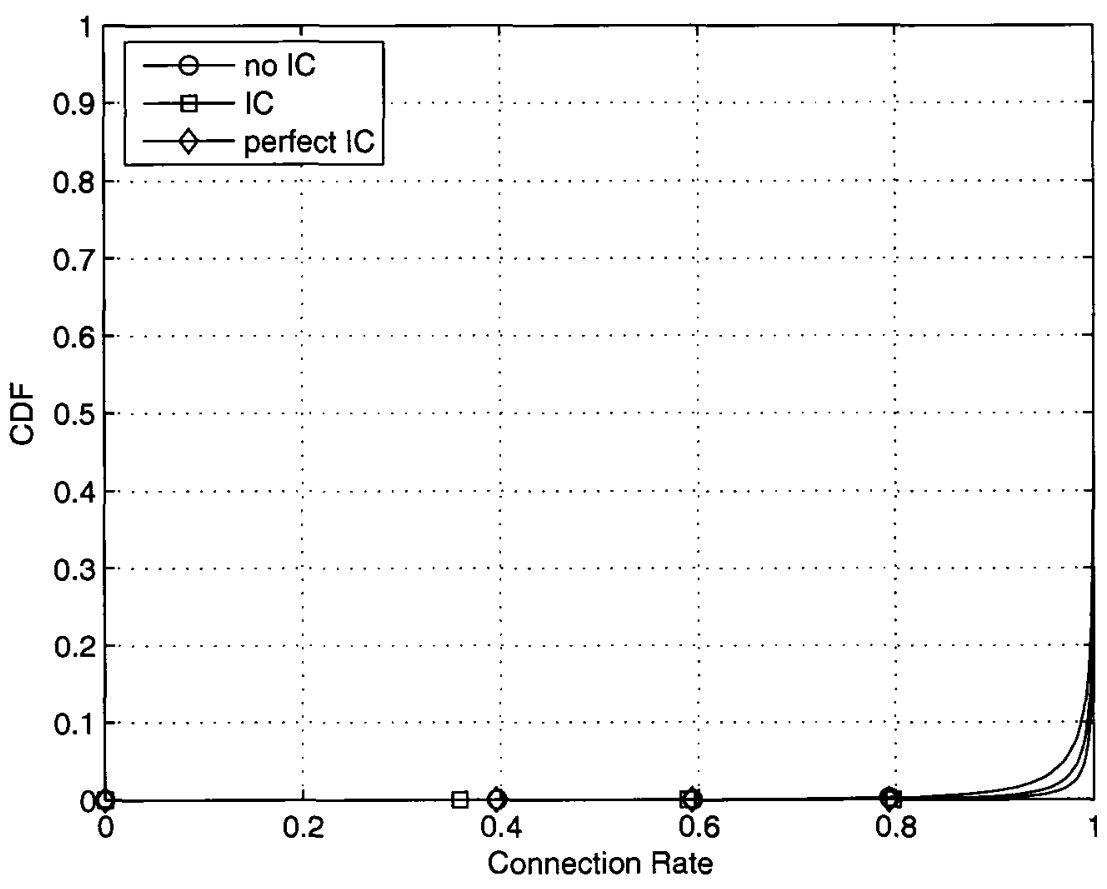

(c) CDF of connection rate.

Figure 4.16: Scheme 366 (cell reuse factor $=3$, sectors $/$ cell $=6$, sector reuse factor $=6$ ). 
able to significantly improve system performance, the design of the frequency reuse layout plays a more decisive role on system performance.

\subsection{Simulation Results of Throughput}

In the previous section we confirmed that increasing the frequency reuse factor was an important way to improve the connection rate (in bits per channel use). For example, Scheme 311 gives much better performance than Scheme 111 because of the reduced CCI in the system. However, in practice, the total system bandwidth is usually fixed, so an increased frequency reuse factor implies that the bandwidth of each frequency group is narrower. As a result, users in Scheme 111 will have three times the bandwidth of users in Scheme 311, and will therefore have higher throughput (in terms of bits per second) than indicated by the connection rate. Furthermore, when sectoring is used, multiple users in a cell can be served simultaneously, as opposed to sequentially (when TDMA-type scheduling is used), resulting in increased throughput to each user. We therefore define the normalized throughput as

$$
\eta=C_{R} \frac{N_{S}}{N_{F}}=C_{R} \frac{N_{S}}{N_{C F} N_{S F}}
$$

where $C_{R}$ is the connection rate, $N_{S}$ is the number of sectors per cell, $N_{F}=N_{C F} N_{S F}$ is the number of frequency groups, $N_{C F}$ is the cell frequency reuse factor, and $N_{S F}$ is the sector frequency reuse factor.

By this way, we produce the CDF of the throughput curves shown in Figure 4.17 and Figure 4.18. The average connection rates and the average normalized throughput of all schemes can be found in Table 4.3 . 


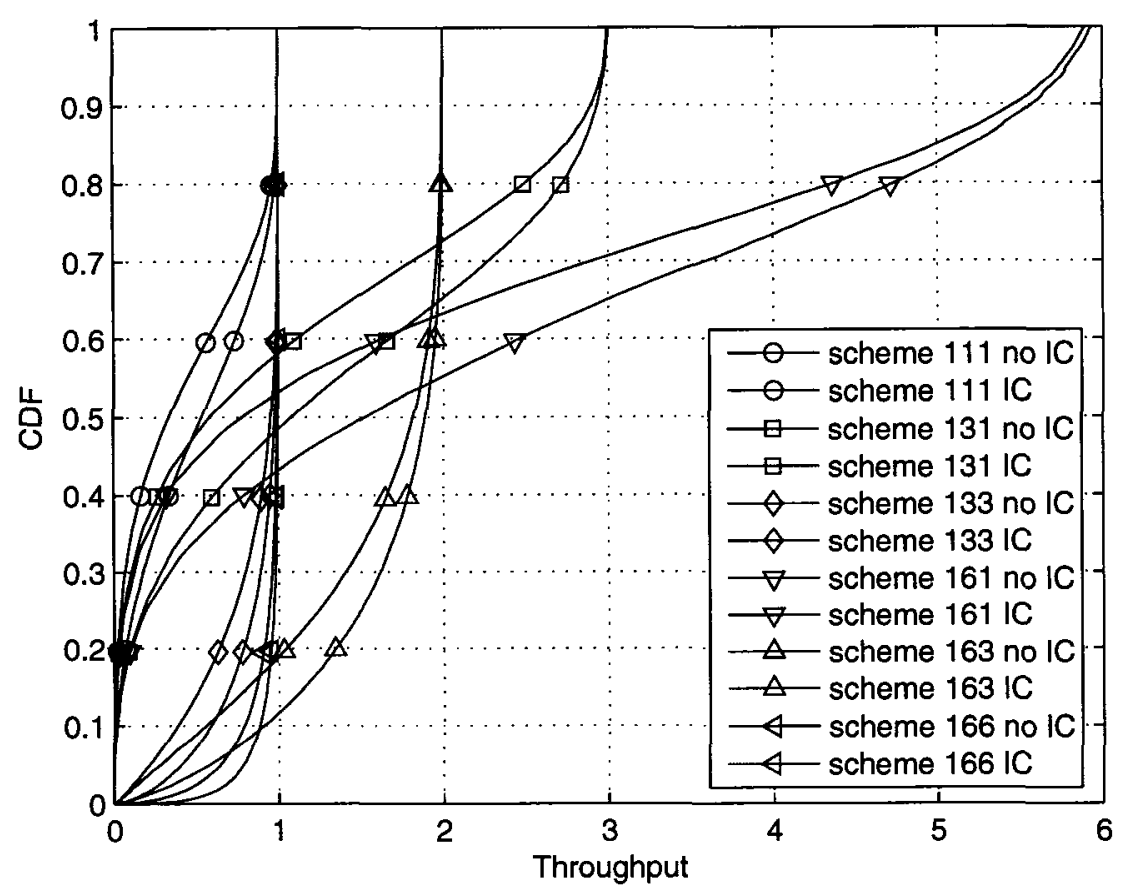

Figure 4.17: CDF-throughput for cell frequency reuse $=1$.

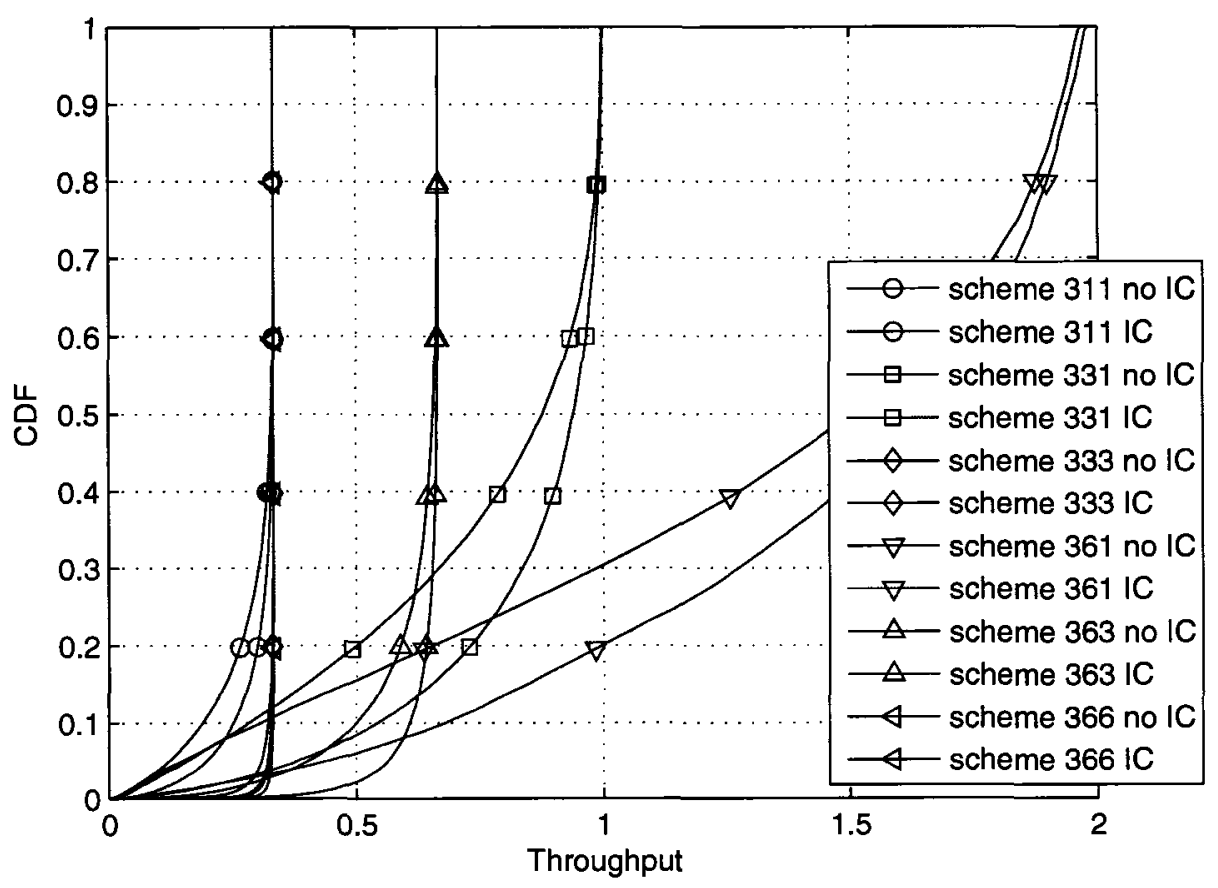

Figure 4.18: CDF-throughput for cell frequency reuse $=3$. 


\begin{tabular}{|l|r|r|r|r|r|r|}
\hline Scheme & $N_{C F}$ & $N_{S}$ & $N_{S F}$ & $\frac{N_{S}}{\left(N_{C F} N_{S F}\right)}$ & $\begin{array}{r}\text { average } \\
\text { connection rate }\end{array}$ & $\begin{array}{r}\text { average } \\
\text { throughput }\end{array}$ \\
\hline \hline 111 & 1 & 1 & 1 & 1 & 0.527 & 0.527 \\
\hline 131 & 1 & 3 & 1 & 3 & 0.443 & 1.329 \\
\hline 133 & 1 & 3 & 3 & 1 & 0.880 & 0.880 \\
\hline 161 & 1 & 6 & 1 & 6 & 0.371 & 2.226 \\
\hline 163 & 1 & 6 & 3 & 2 & 0.832 & 1.664 \\
\hline 166 & 1 & 6 & 6 & 1 & 0.961 & 0.961 \\
\hline 311 & 3 & 1 & 1 & $1 / 3$ & 0.929 & 0.310 \\
\hline 331 & 3 & 3 & 1 & 1 & 0.851 & 0.851 \\
\hline 333 & 3 & 3 & 3 & $1 / 3$ & 0.993 & 0.331 \\
\hline 361 & 3 & 6 & 1 & 2 & 0.733 & 1.466 \\
\hline 363 & 3 & 6 & 3 & $2 / 3$ & 0.970 & 0.647 \\
\hline 366 & 3 & 6 & 6 & $1 / 3$ & 0.997 & 0.332 \\
\hline
\end{tabular}

Table 4.3: Average connection rate and average throughput, IC case.

We notice that the scheme with the highest average connection rate (Scheme 366, Figure 4.16) has the lowest throughput, because the large cell and sector reuse factors lower the average bandwidth per user. Thus the bandwidth is not effectively used.

The highest average throughput comes from the most aggressive scheme, Scheme 161 (Figure 4.7). However, we notice from Figure 4.17 that about $10 \%$ of the users have virtually no throughput, which could be intolerable for a practical system. Scheme 111 (Figure 4.5) and Scheme 131 (Figure 4.6) also have the identical problem. The common character of these three schemes is that no frequency reuse is applied $\left(N_{C F}=N_{S F}=1\right)$. Such aggressive designs induce tremendous CCI.

After removing the three schemes above, Scheme 163 (Figure 4.9) has the highest average throughput, and Scheme 361 (Figure 4.14) ranks the second. The two schemes have the same sectoring and the same frequency groups (A, B and $\mathrm{C})$, but different 
distribution patterns. Scheme 163 has a more reasonable pattern and achieves better throughput. The two schemes also tells us that sectoring is an effective way to reduce CCI and improve the throughput, and that the 6-sector structure is better than 3sector structure.

Figure 4.19 shows the CDF of the normalized throughput for Scheme 163, for the "no IC", "IC", and "perfect IC" cases. We note that the IC increases the average throughput from 1.542 to 1.664 (for an increase of $7.9 \%$ ), the median throughput from 1.81 to 1.886 (for an increase of $2.8 \%$ ), and the $10 \%$ outage throughput from 0.55 to 0.894 (for an increase of $62.6 \%$ ). The 0.4 outage probability drops from $7.1 \%$ to $2.9 \%$.

Although the IC is not very helpful at increasing the average or median throughput, its advantages in terms of the outage throughput and outage probability are significant. And if there are strict requirements placed on the minimum acceptable outage probability (or outage throughput), then the use of IC may allow for the use of a more aggressive frequency reuse scheme that would otherwise be possible, allowing for significant gains in throughput. For example, if it is required that no more than $5 \%$ of the users have a normalized throughput of less than 0.5 , then, without the IC, the best possible scheme is Scheme 166, which gives an average throughput of 0.961 . However, with the IC, Scheme 163 can be used instead, with an average throughput of 1.664 . 


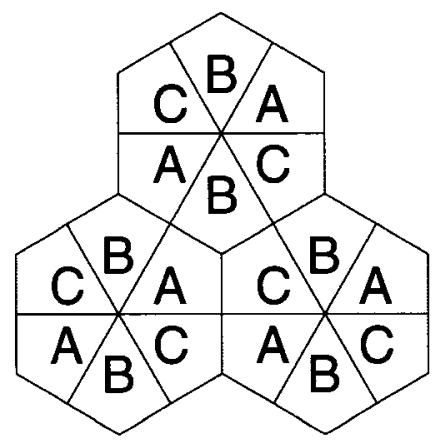

(a) Layout.

\begin{tabular}{|l|c|c|c|}
\hline & no IC & IC & $\begin{array}{c}\text { perfect } \\
\text { IC }\end{array}$ \\
\hline \hline Ave. throughput & 1.542 & 1.664 & 1.746 \\
\hline Median throughput & 1.810 & 1.886 & 1.932 \\
\hline $10 \%$ outage rate & 0.550 & 0.894 & 1.144 \\
\hline 0.4 outage probability & 0.071 & 0.029 & 0.015 \\
\hline
\end{tabular}

(b) Statistics.

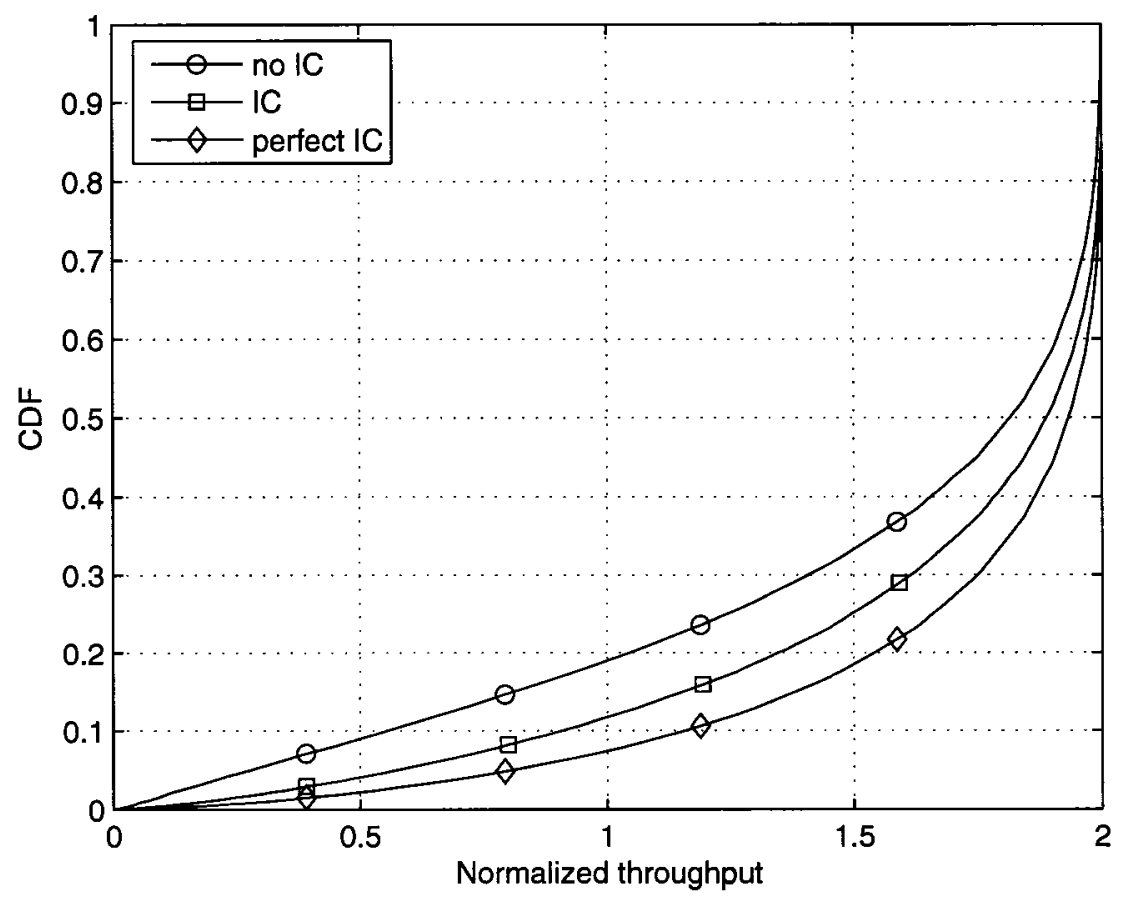

(c) CDF of normalized throughput.

Figure 4.19: CDF of normalized throughput for Scheme 163. 


\section{Chapter 5}

\section{Conclusions and Future Work}

\subsection{Conclusions}

In this thesis, we generalize an iterative MIMO detector to be applicable to fully asynchronous systems. In our model, several transmitted blocks form a transmitted frame such that the offset caused by the asynchronization among multiple users only affects the decoding of the first and last blocks of the frame. The offset symbols are truncated from the frames to pretend to be the synchronous data. We also derive the algorithm which is applicable for the truncated frames. The random delay of every ISI tap is used to realize the asyncronization among symbols. The conclusions of simulation results are as follows:

- The simulation over the SUI-5 channel, the 5-tap channel, and the Long channel proves that our asynchronous model can effectively eliminate ISI and CCI if the receive antenna number is large enough. 
- The BER and WER performance from simulation shows that our fully asynchronous model suffer minor BER penalty compared to the synchronous model.

- The 3-tier cellular model simulation shows that our model can effectively eliminate the primary CCI for various cellular layouts. The IC technique can significantly improve the system connection rate and the system throughput.

- The cellular simulation also proves that the layout design is very important to system performance.

\subsection{Future Work}

- In our model, we assume that the channel characters are known to the receiver. The adaptive channel estimation, particularly at the beginning of a call or during handoffs, is a vital aspect of future work for iterative MIMO detectors.

- In our model, the cyclic prefix between two neighboring blocks is removed to avoid the BER "floor" such that the linear convolution instead of circular convolution is used in frequency-domain filtering. How to obtain the performance and the efficient calculation at the same time could be a future work.

- Lesson: the oversampling can not improve the BER performance. This can NOT be a future work. 


\section{Appendix A}

\section{Detector Parameter Calculation}

In this appendix, we derive the detector filtering coefficients $P_{k}^{(s, a)}$ and $b_{n}^{(s)}$ (Equation 2.9, Page 21), which we omit in Subsection 2.3.1 (Page 20). We also calculate the mean and the variance of estimate transmitted symbol $z_{n}^{(s)}$. In order to avoid the computational instability, some adjustments are introduced at the end of this appendix.

\section{A.1 Detector Coefficients Derivation}

The derivation begins with Equation 2.9 (Page 21), which shows the output of the filter for user $s$ at time $n$

$$
z_{n}^{(s)}=\frac{1}{N_{T}} \sum_{k=0}^{N_{T}-1} \sum_{a=1}^{N_{A}} P_{k}^{(s, a)} R_{k}^{(a)} \mathrm{e}^{j 2 \pi \frac{k n}{N_{T}}}+b_{n}^{(s)}
$$

where $n \in\left\{0,1,2, \ldots, N_{T}-1\right\}, s \in\left\{1,2, \ldots, N_{S}\right\}$ and $a \in\left\{1,2, \ldots, N_{A}\right\}$. 
In order to find the proper $P_{k}^{(s, a)}$ and $b_{n}^{(s)}$, we apply minimum mean square error (MMSE) criterion to the mean square error between $z_{n}^{(s)}$ and $v_{n}^{(s)}$

$$
J^{(s)}=\frac{1}{N_{T}} \sum_{n=0}^{N_{T}-1} \mathcal{E}\left[\left|z_{n}^{(s)}-v_{n}^{(s)}\right|^{2}\right] .
$$

Furthermore, to guarantee $P_{k}^{(s, a)}$ time-invariant, we may deploy the time-averaged MSE criterion.

In order to obtain the detector coefficients for user $s$, we may apply $J^{(s)}$ partial derivatives with respect to $P_{k}^{(s, a)}$ and $J^{(s)}$ for all $k$ and $n$, and let these derivatives be zero.

Because $\partial J^{(s)} / \partial b_{n}^{(s)}=0$ is equivalent to

$$
\frac{1}{N_{T}} \sum_{m=0}^{N_{T}-1} \mathcal{E}\left[\left(z_{m}^{(s)}-v_{m}^{(s)}\right) \frac{\partial}{\partial b_{n}^{(s)}}\left(z_{m}^{(s)}-v_{m}^{(s)}\right)^{*}\right]=0
$$

and

$$
\begin{aligned}
\frac{\partial}{\partial b_{n}^{(s)}}\left(z_{m}^{(s)}-v_{m}^{(s)}\right) & =\frac{\partial}{\partial b_{n}^{(s)}} z_{m}^{(s)} \\
& =\delta_{n-m},
\end{aligned}
$$

Equation A.3 becomes

$$
\frac{1}{N_{T}} \sum_{m=0}^{N_{T^{-1}}} \mathcal{E}\left[\left(z_{m}^{(s)}-v_{m}^{(s)}\right) \delta_{n-m}\right]=0 .
$$

Thus

$$
\begin{aligned}
\bar{z}_{n}^{(s)} & =\mathcal{E}\left[z_{n}^{(s)}\right] \\
& =\mathcal{E}\left[v_{n}^{(s)}\right] \\
& =\bar{v}_{n}^{(s)} .
\end{aligned}
$$


Therefore the expectation-version of Equation A.1 may be written as

$$
\bar{v}_{n}^{(s)}=\frac{1}{N_{T}} \sum_{k=0}^{N_{T}-1} \sum_{a=1}^{N_{A}} P_{k}^{(s, a)} \mathcal{E}\left[R_{k}^{(a)}\right] \mathrm{e}^{j 2 \pi \frac{k n}{N_{T}}}+b_{n}^{(s)},
$$

or equivalently

$$
b_{n}^{(s)}=\bar{v}_{n}^{(s)}-\frac{1}{N_{T}} \sum_{k=0}^{N_{T}-1} \sum_{a=1}^{N_{A}} P_{k}^{(s, a)} \bar{R}_{k}^{(a)} \mathrm{e}^{j 2 \pi \frac{k n}{N_{T}}} .
$$

Substituting Equation A.8 to Equation A.1, we can obtain the detector output

$$
z_{n}^{(s)}=\frac{1}{N_{T}} \sum_{k=0}^{N_{T}-1} \sum_{a=1}^{N_{A}} P_{k}^{(s, a)}\left(R_{k}^{(a)}-\bar{R}_{k}^{(a)}\right) \mathrm{e}^{j 2 \pi \frac{k n}{N_{T}}}+\bar{v}_{n}^{(s)} .
$$

Similarly, since $\partial J^{(s)} / \partial P_{l}^{(s, b)}=0$ is equivalent to

$$
\frac{1}{N_{T}} \sum_{n=0}^{N_{T}-1} \mathcal{E}\left[\left(z_{n}^{(s)}-v_{n}^{(s)}\right) \frac{\partial}{\partial P_{l}^{(s, b)}}\left(z_{n}^{(s)}-v_{n}^{(s)}\right)^{*}\right]=0
$$

and

$$
\frac{\partial}{\partial P_{l}^{(s, b)}}\left(z_{n}^{(s)}-v_{n}^{(s)}\right)=\frac{1}{N_{T}}\left(R_{l}^{(b)}-\bar{R}_{l}^{(b)}\right) \mathrm{e}^{j 2 \pi \frac{l n}{N_{T}}}
$$

from Equation A.10 we can obtain

$$
\frac{1}{N_{T}} \sum_{n=0}^{N_{T}-1} \mathcal{E}\left[\left(z_{n}^{(s)}-v_{n}^{(s)}\right) \frac{1}{N_{T}}\left(R_{l}^{(b)}-\bar{R}_{l}^{(b)}\right)^{*} \mathrm{e}^{-j 2 \pi \frac{l n}{N_{T}}}\right]=0
$$

Substituting $z_{n}^{(s)}$ with Equation A.9, we can obtain

$$
\begin{array}{r}
\frac{1}{N_{T}} \sum_{n=0}^{N_{T}-1} \mathcal{E}\left[\left(\frac{1}{N_{T}} \sum_{k=0}^{N_{T}-1} \sum_{a=1}^{N_{A}} P_{k}^{(s, a)}\left(R_{k}^{(a)}-\bar{R}_{k}^{(a)}\right) \mathrm{e}^{j 2 \pi \frac{k n}{N_{T}}}+\bar{v}_{n}^{(s)}-v_{n}^{(s)}\right)\right. \\
\left.\times\left(R_{l}^{(b)}-\bar{R}_{l}^{(b)}\right)^{*} \mathrm{e}^{-j 2 \pi \frac{l n}{N_{T}}}\right]=0
\end{array}
$$

which can be expressed as

$$
\begin{array}{r}
\frac{1}{\left(N_{T}\right)^{2}} \sum_{n=0}^{N_{T}-1} \sum_{k=0}^{N_{T}-1} \sum_{a=1}^{N_{A}} P_{k}^{(s, a)} \mathcal{E}\left[\left(R_{k}^{(a)}-\bar{R}_{k}^{(a)}\right)\left(R_{l}^{(b)}-\bar{R}_{l}^{(b)}\right)^{*}\right] \mathrm{e}^{j 2 \pi \frac{(k-l) n}{N_{T}}} \\
=\frac{1}{N_{T}} \sum_{n=0}^{N_{T}-1} \mathcal{E}\left[\left(v_{n}^{(s)}-\bar{v}_{n}^{(s)}\right)\left(R_{l}^{(b)}-\bar{R}_{l}^{(b)}\right)^{*}\right] \mathrm{e}^{-j 2 \pi \frac{l n}{N_{T}}}
\end{array}
$$


which can be simplified as

$$
\begin{aligned}
\frac{1}{N_{T}} \sum_{k=0}^{N_{T}-1} \sum_{a=1}^{N_{A}} P_{k}^{(s, a)} & \operatorname{Cov}\left[R_{k}^{(a)}, R_{l}^{(b)}\right] \frac{1}{N_{T}} \sum_{n=0}^{N_{T}-1} \mathrm{e}^{j 2 \pi \frac{(k-l) n}{N_{T}}} \\
= & \frac{1}{N_{T}} \sum_{n=0}^{N_{T}-1} \operatorname{Cov}\left[v_{n}^{(s)}, R_{l}^{(b)}\right] \mathrm{e}^{-j 2 \pi \frac{l n}{N_{T}}}
\end{aligned}
$$

where the symbol "Cov" is defined as $\operatorname{Cov}[X, Y]=\mathcal{E}\left[(X-\mathcal{E}[X])(Y-\mathcal{E}[Y])^{*}\right]$. Because

$$
\frac{1}{N_{T}} \sum_{n=0}^{N_{T}-1} \mathrm{e}^{j 2 \pi \frac{k n}{N_{T}}}=\delta_{k}
$$

Equation A.15 can be simplified as

$$
\begin{array}{r}
\frac{1}{N_{T}} \sum_{k=0}^{N_{T}-1} \sum_{a=1}^{N_{A}} P_{k}^{(s, a)} \operatorname{Cov}\left[R_{k}^{(a)}, R_{l}^{(b)}\right] \delta_{k-l} \\
=\frac{1}{N_{T}} \sum_{n=0}^{N_{T}-1} \operatorname{Cov}\left[v_{n}^{(s)}, R_{l}^{(b)}\right] \mathrm{e}^{-j 2 \pi \frac{l n}{N_{T}}}
\end{array}
$$

thus

$$
\frac{1}{N_{T}} \sum_{a=1}^{N_{A}} P_{l}^{(s, a)} \operatorname{Cov}\left[R_{l}^{(a)}, R_{l}^{(b)}\right]=\frac{1}{N_{T}} \sum_{n=0}^{N_{T}-1} \operatorname{Cov}\left[v_{n}^{(s)}, R_{l}^{(b)}\right] \mathrm{e}^{-j 2 \pi \frac{l n}{N_{T}}}
$$

Before we continue to simplify Equation A.18, we have to calculate the covariances first. Because of the independence of the transmitted symbols of different users or at different times, we can simplify the covariance between $v_{n}^{(s)}$ and $v_{m}^{(u)}$ as follows

$$
\begin{aligned}
& \operatorname{Cov}\left[v_{n}^{(s)}, v_{m}^{(u)}\right] \\
& =\mathcal{E}\left[\left(v_{n}^{(s)}-\bar{v}_{n}^{(s)}\right)\left(v_{m}^{(u)}-\bar{v}_{m}^{(u)}\right)^{*}\right] \\
& = \begin{cases}\mathcal{E}\left[v_{n}^{(s)}-\bar{v}_{n}^{(s)}\right] \mathcal{E}\left[v_{m}^{(u)}-\bar{v}_{m}^{(u)}\right]^{*} & \text { if } m \neq n \text { or } s \neq u \\
\mathcal{E}\left[\left|v_{n}^{(s)}-\bar{v}_{n}^{(s)}\right|^{2}\right] & \text { if } m=n \text { and } s=u\end{cases} \\
& = \begin{cases}0 & \text { if } m \neq n \text { or } s \neq u \\
\sigma_{v, n, s}^{2} & \text { if } m=n \text { and } s=u\end{cases} \\
& =\sigma_{v, n, s}^{2} \delta_{m-n} \delta_{s-u},
\end{aligned}
$$


where $\sigma_{v, n, s}^{2}=\mathcal{E}\left[\left|v_{n}^{(s)}-\bar{v}_{n}^{(s)}\right|^{2}\right]$ is the variance of $v_{n}^{(s)}$.

Because

$$
V_{k}^{(s)}=\sum_{n=0}^{N_{T}-1} v_{n} \mathrm{e}^{-j 2 \pi \frac{k n}{N_{T}}}
$$

is the DFT of $v_{n}$, we can simplify the covariance between $v_{n}^{(s)}$ and $V_{k}^{(s)}$ as follows

$$
\begin{aligned}
\operatorname{Cov}\left[v_{n}^{(s)}, V_{l}^{(u)}\right] & =\mathcal{E}\left[\left(v_{n}^{(s)}-\bar{v}_{n}^{(s)}\right)\left(V_{l}^{(u)}-\bar{V}_{l}^{(u)}\right)^{*}\right] \\
& =\sum_{m=0}^{N_{T}-1} \mathcal{E}\left[\left(v_{n}^{(s)}-\bar{v}_{n}^{(s)}\right)\left(v_{m}^{(u)}-\bar{v}_{m}^{(u)}\right)^{*}\right] \mathrm{e}^{j 2 \pi \frac{l m}{N_{T}}} \\
& =\sum_{m=0}^{N_{T}-1} \operatorname{Cov}\left[v_{n}^{(s)}, v_{m}^{(u)}\right] \mathrm{e}^{j 2 \pi \frac{l m}{N_{T}}} \\
& =\sum_{m=0}^{N_{T}-1}\left[\sigma_{v, n, s}^{2} \delta_{m-n} \delta_{s-u}\right] \mathrm{e}^{j 2 \pi \frac{l m}{N_{T}}} \\
& =\sigma_{v, n, s}^{2} \delta_{s-u} \mathrm{e}^{j 2 \pi \frac{l n}{N_{T}}} .
\end{aligned}
$$

Because

$$
R_{k}^{(a)}=\sum_{u=1}^{N_{S}} H_{k}^{(u, a)} V_{k}^{(u)}+W_{k}^{(a)}
$$

is the DFT of the received samples from antenna $a$, we can simplify the covariance between $v_{n}^{(s)}$ and $R_{l}^{(b)}$ as follows

$$
\begin{aligned}
\operatorname{Cov}\left[v_{n}^{(s)}, R_{l}^{(b)}\right] \\
=\mathcal{E}\left[\left(v_{n}^{(s)}-\bar{v}_{n}^{(s)}\right)\left(R_{l}^{(b)}-\bar{R}_{l}^{(b)}\right)^{*}\right] \\
=\mathcal{E}\left[\left(v_{n}^{(s)}-\bar{v}_{n}^{(s)}\right)\left(\sum_{u=1}^{N_{S}} H_{l}^{(u, b)} V_{l}^{(u)}+W_{l}^{(b)}-\sum_{u=1}^{N_{S}} H_{l}^{(u, b)} \bar{V}_{l}^{(u)}\right)^{*}\right] \\
=\sum_{u=1}^{N_{S}} H_{l}^{(u, b) *} \mathcal{E}\left[\left(v_{n}^{(s)}-\bar{v}_{n}^{(s)}\right)\left(V_{l}^{(u)}-\bar{V}_{l}^{(u)}\right)^{*}\right] \\
\quad+\mathcal{E}\left[\left(v_{n}^{(s)}-\bar{v}_{n}^{(s)}\right) W_{l}^{(b) *}\right] \\
=\sum_{u=1}^{N_{S}} H_{l}^{(u, b) *} \operatorname{Cov}\left[v_{n}^{(s)}, V_{l}^{(u)}\right]+\mathcal{E}\left[\left(v_{n}^{(s)}-\bar{v}_{n}^{(s)}\right)\right] \mathcal{E}\left[W_{l}^{(b) *}\right]
\end{aligned}
$$




$$
\begin{aligned}
& =\sum_{u=1}^{N_{S}} H_{l}^{(u, b) *}\left[\sigma_{v, n, s}^{2} \delta_{s-u} \mathrm{e}^{j 2 \pi \frac{l n}{N_{T}}}\right] \\
& =H_{l}^{(s, b) *} \sigma_{v, n, s}^{2} \mathrm{e}^{j 2 \pi \frac{l n}{N_{T}}} .
\end{aligned}
$$

Therefore, the covariance between $V_{k}^{(s)}$ and $R_{l}^{(b)}$ can be expressed as

$$
\begin{aligned}
\operatorname{Cov}\left[V_{k}^{(s)}, R_{l}^{(b)}\right] & =\mathcal{E}\left[\left(V_{k}^{(s)}-\bar{V}_{k}^{(s)}\right)\left(R_{l}^{(b)}-\bar{R}_{l}^{(b)}\right)^{*}\right] \\
& =\sum_{m=0}^{N_{T}-1} \mathcal{E}\left[\left(v_{m}^{(s)}-\bar{v}_{m}^{(s)}\right)\left(R_{l}^{(b)}-\bar{R}_{l}^{(b)}\right)^{*}\right] \mathrm{e}^{-j 2 \pi \frac{k m}{N_{T}}} \\
& =\sum_{m=0}^{N_{T}-1} \operatorname{Cov}\left[v_{m}^{(s)}, R_{l}^{(b)}\right] \mathrm{e}^{-j 2 \pi \frac{k m}{N_{T}}} \\
& =\sum_{m=0}^{N_{T}-1}\left[H_{l}^{(s, b) *} \sigma_{v, m, s}^{2} \mathrm{e}^{j 2 \pi \frac{l m}{N_{T}}}\right] \mathrm{e}^{-j 2 \pi \frac{k m}{N_{T}}} \\
& =H_{l}^{(s, b) *} \sum_{m=0}^{N_{T}-1} \sigma_{v, m, s}^{2} \mathrm{e}^{-j 2 \pi \frac{(k-l) m}{N_{T}}}
\end{aligned}
$$

and the variance between $R_{k}^{(a)}$ and $R_{l}^{(b)}$ as

$$
\begin{aligned}
\operatorname{Cov}\left[R_{k}^{(a)}, R_{l}^{(b)}\right] \\
=\mathcal{E}\left[\left(R_{k}^{(a)}-\bar{R}_{k}^{(a)}\right)\left(R_{l}^{(b)}-\bar{R}_{l}^{(b)}\right)^{*}\right] \\
=\mathcal{E}\left[\left(\sum_{s=1}^{N_{S}} H_{k}^{(s, a)} V_{k}^{(s)}+W_{k}^{(a)}-\sum_{s=1}^{N_{S}} H_{k}^{(s, a)} \bar{V}_{k}^{(s)}\right)\left(R_{l}^{(b)}-\bar{R}_{l}^{(b)}\right)^{*}\right] \\
=\sum_{s=1}^{N_{S}} H_{k}^{(s, a)} \operatorname{Cov}\left[V_{k}^{(s)}, R_{l}^{(b)}\right]+\mathcal{E}\left[W_{k}^{(a)}\left(R_{l}^{(b)}-\bar{R}_{l}^{(b)}\right)^{*}\right] \\
=\sum_{s=1}^{N_{S}} H_{k}^{(s, a)}\left(H_{l}^{(s, b) *} \sum_{m=0}^{N_{T}-1} \sigma_{v, m, s}^{2} \mathrm{e}^{-j 2 \pi \frac{(k-l) m}{N_{T}}}\right) \\
\quad+\sum_{u=1}^{N_{S}} H_{l}^{(u, b) *} \mathcal{E}\left[W_{k}^{(a)}\left(V_{l}^{(b)}-\bar{V}_{l}^{(b)}\right)^{*}\right]+\mathcal{E}\left[W_{k}^{(a)} W_{l}^{(b) *}\right] \\
=\sum_{s=1}^{N_{S}} H_{k}^{(s, a)} H_{l}^{(s, b) *} \sum_{m=0}^{N_{T}-1} \sigma_{v, m, s}^{2} \mathrm{e}^{-j 2 \pi \frac{(k-l) m}{N_{T}}}+N_{T} N_{0} \delta_{k-l} \delta_{a-b} .
\end{aligned}
$$

Since we have known $\operatorname{Cov}\left[v_{n}^{(s)}, R_{l}^{(b)}\right]$ and $\operatorname{Cov}\left[R_{k}^{(a)}, R_{l}^{(b)}\right]$ from Equation A.23 and Equation A.25 respectively, we can continue the calculation in Equation A.18 (Page 
98) as follows

$$
\begin{array}{r}
\frac{1}{N_{T}} \sum_{a=1}^{N_{A}} P_{l}^{(s, a)}\left[\sum_{u=1}^{N_{S}} H_{l}^{(u, a)} H_{l}^{(u, b) *} \sum_{m=0}^{N_{T}-1} \sigma_{v, m, u}^{2}+N_{T} N_{0} \delta_{a-b}\right] \\
=\frac{1}{N_{T}} \sum_{n=0}^{N_{T}-1}\left[H_{l}^{(s, b) *} \sigma_{v, n, s}^{2} \mathrm{e}^{j 2 \pi \frac{l n}{N_{T}}}\right] \mathrm{e}^{-j 2 \pi \frac{l n}{N_{T}}}
\end{array}
$$

thus

$$
\begin{array}{r}
\sum_{a=1}^{N_{A}} P_{l}^{(s, a)}\left[\sum_{u=1}^{N_{S}} H_{l}^{(u, a)} H_{l}^{(u, b) *}\left(\frac{1}{N_{T}} \sum_{m=0}^{N_{T}-1} \sigma_{v, m, u}^{2}\right)+N_{0} \delta_{a-b}\right] \\
=H_{l}^{(s, b) *} \frac{1}{N_{T}} \sum_{n=0}^{N_{T}-1} \sigma_{v, n, s}^{2}
\end{array}
$$

which can also be expressed as

$$
\sum_{a=1}^{N_{A}} P_{l}^{(s, a)}\left[\sum_{u=1}^{N_{S}} H_{l}^{(u, a)} H_{l}^{(u, b) *} \bar{\sigma}_{v, u}^{2}+N_{0} \delta_{a-b}\right]=H_{l}^{(s, b) *} \bar{\sigma}_{v, s}^{2}
$$

where

$$
\bar{\sigma}_{v, s}^{2}=\frac{1}{N_{T}} \sum_{n=0}^{N_{T}-1} \sigma_{v, n, s}^{2}
$$

By defining

$$
\Gamma_{l}^{(b, a)}=\sum_{u=1}^{N_{S}} H_{l}^{(u, a)} H_{l}^{(u, b) *} \bar{\sigma}_{v, u}^{2}+N_{0} \delta_{a-b},
$$

Equation A.28 can be expressed in matrix form as

$$
\left[\begin{array}{cccc}
\Gamma_{l}^{(1,1)} & \Gamma_{l}^{(1,2)} & \ldots & \Gamma_{l}^{\left(1, N_{A}\right)} \\
\Gamma_{l}^{(2,1)} & \Gamma_{l}^{(2,2)} & \ldots & \Gamma_{l}^{\left(2, N_{A}\right)} \\
\vdots & \vdots & \ddots & \vdots \\
\Gamma_{l}^{\left(N_{A}, 1\right)} & \Gamma_{l}^{\left(N_{A}, 2\right)} & \ldots & \Gamma_{l}^{\left(N_{A}, N_{A}\right)}
\end{array}\right]\left[\begin{array}{c}
P_{l}^{(s, 1)} \\
P_{l}^{(s, 2)} \\
\vdots \\
P_{l}^{\left(s, N_{A}\right)}
\end{array}\right]=\bar{\sigma}_{v, s}^{2}\left[\begin{array}{c}
H_{l}^{(s, 1) *} \\
H_{l}^{(s, 2) *} \\
\vdots \\
H_{l}^{\left(s, N_{A}\right) *}
\end{array}\right]
$$


where the detector coefficients $P_{l}^{(s, b)}$ can be calculated as

$$
\left[\begin{array}{c}
P_{l}^{(s, 1)} \\
P_{l}^{(s, 2)} \\
\vdots \\
P_{l}^{\left(s, N_{A}\right)}
\end{array}\right]=\bar{\sigma}_{v, s}^{2}\left[\begin{array}{cccc}
\Gamma_{l}^{(1,1)} & \Gamma_{l}^{(1,2)} & \cdots & \Gamma_{l}^{\left(1, N_{A}\right)} \\
\Gamma_{l}^{(2,1)} & \Gamma_{l}^{(2,2)} & \cdots & \Gamma_{l}^{\left(2, N_{A}\right)} \\
\vdots & \vdots & \ddots & \vdots \\
\Gamma_{l}^{\left(N_{A}, 1\right)} & \Gamma_{l}^{\left(N_{A}, 2\right)} & \cdots & \Gamma_{l}^{\left(N_{A}, N_{A}\right)}
\end{array}\right]^{-1}\left[\begin{array}{c}
H_{l}^{(s, 1) *} \\
H_{l}^{(s, 2) *} \\
\vdots \\
H_{l}^{\left(s, N_{A}\right) *}
\end{array}\right]
$$

for every $l \in\left\{0,1, \ldots, N_{T}-1\right\}$.

The detector coefficients $b_{n}^{(s)}$ can be calculated by Equation A.8 (Page 97) for every $n \in\left\{0,1, \ldots, N_{T}-1\right\}$.

\section{A.2 Mean and Variance of $z_{n}^{(s)}$}

For every hypothetical $d \in\{0,1, \ldots, M-1\}$, the branch metrics may be expressed as Equation 2.13 (Page 23)

$$
\gamma_{n}^{(s)}(d)=f\left(z_{n}^{(s)} \mid d_{n}^{(s)}=d\right)
$$

In practice, since the interference in $z_{n}^{(s)}$ can be considered as Gaussian, the branch metrics may also be expressed in Gaussian form

$$
\gamma_{n}^{(s)}(d)=\frac{1}{\pi \sigma_{z, n, s}^{2}} \exp \left\{-\frac{1}{\sigma_{z, n, s}^{2}}\left|z_{n}^{(s)}-\mu_{n}^{(s)}(d)\right|^{2}\right\},
$$

where

$$
\mu_{n}^{(s)}(d)=\mathcal{E}\left[z_{n}^{(s)} \mid d_{n}^{(s)}=d\right]
$$

is the mean of $z_{n}^{(s)}$ under the condition that $d_{n}^{(s)}=d$, and

$$
\sigma_{z, n, s}^{2}=\mathcal{E}\left[\left|z_{n}^{(s)}-\mu_{n}^{(s)}(d)\right|^{2} \mid d_{n}^{(s)}=d\right]
$$


is the corresponding variance.

From Equation (A.9), we know

$$
\begin{aligned}
& \mu_{n}^{(s)}(d) \\
& =\frac{1}{N_{T}} \sum_{k=0}^{N_{T}-1} \sum_{a=1}^{N_{A}} P_{k}^{(s, a)}\left(\mathcal{E}\left[R_{k}^{(a)} \mid d_{n}^{(s)}=d\right]-\bar{R}_{k}^{(a)}\right) \mathrm{e}^{j 2 \pi \frac{k n}{N_{T}}}+\bar{v}_{n}^{(s)} .
\end{aligned}
$$

In order to calculate $\mathcal{E}\left[R_{k}^{(a)} \mid d_{n}^{(s)}=d\right]$, we may first calculate

$$
\begin{aligned}
\mathcal{E}\left[v_{m}^{(u)} \mid d_{n}^{(s)}=d\right] & = \begin{cases}\mathcal{E}\left[v_{m}^{(u)}\right] & \text { if } m \neq n \text { or } s \neq u \\
\mathcal{E}\left[v_{n}^{(s)} \mid d_{n}^{(s)}=d\right] & \text { if } m=n \text { and } s=u\end{cases} \\
& = \begin{cases}\bar{v}_{m}^{(u)} & \text { if } m \neq n \text { or } s \neq u \\
\operatorname{SM}[d] & \text { if } m=n \text { and } s=u\end{cases} \\
& =\bar{v}_{m}^{(u)}\left(1-\delta_{m-n} \delta_{s-u}\right)+\operatorname{SM}[d] \delta_{m-n} \delta_{s-u} \\
& =\bar{v}_{m}^{(u)}+\left(\operatorname{SM}[d]-\bar{v}_{n}^{(s)}\right) \delta_{m-n} \delta_{s-u}
\end{aligned}
$$

Thus

$$
\begin{aligned}
\mathcal{E} & {\left[R_{k}^{(a)} \mid d_{n}^{(s)}=d\right] } \\
& =\sum_{u=1}^{N_{S}} H_{k}^{(u, a)} \mathcal{E}\left[V_{k}^{(u)} \mid d_{n}^{(s)}=d\right]+\mathcal{E}\left[W_{k}^{(a)} \mid d_{n}^{(s)}=d\right] \\
& =\sum_{u=1}^{N_{S}} H_{k}^{(u, a)} \sum_{m=0}^{N_{T}-1} \mathcal{E}\left[v_{m}^{(u)} \mid d_{n}^{(s)}=d\right] \mathrm{e}^{-j 2 \pi \frac{k m}{N_{T}}}+\mathcal{E}\left[W_{k}^{(a)}\right] \\
= & \sum_{u=1}^{N_{S}} H_{k}^{(u, a)} \sum_{m=0}^{N_{T}-1}\left[\bar{v}_{m}^{(u)}+\left(\mathrm{SM}[d]-\bar{v}_{n}^{(s)}\right) \delta_{m-n} \delta_{s-u}\right] \mathrm{e}^{-j 2 \pi \frac{k m}{N_{T}}} \\
= & \sum_{u=1}^{N_{S}} H_{k}^{(u, a)} \sum_{m=0}^{N_{T}-1} \bar{v}_{m}^{(u)} \mathrm{e}^{-j 2 \pi \frac{k m}{N_{T}}} \\
& +\left(\mathrm{SM}[d]-\bar{v}_{n}^{(s)}\right) \sum_{u=1}^{N_{S}} H_{k}^{(u, a)} \sum_{m=0}^{N_{T}-1} \delta_{m-n} \delta_{s-u} \mathrm{e}^{-j 2 \pi \frac{k m}{N_{T}}} \\
= & \sum_{u=1}^{N_{S}} H_{k}^{(u, a)} \bar{V}_{k}^{(u)}+\left(\mathrm{SM}[d]-\bar{v}_{n}^{(s)}\right) H_{k}^{(s, a)} \mathrm{e}^{-j 2 \pi \frac{k n}{N_{T}}} \\
= & \bar{R}_{k}^{(a)}+\left(\operatorname{SM}[d]-\bar{v}_{n}^{(s)}\right) H_{k}^{(s, a)} \mathrm{e}^{-j 2 \pi \frac{k n}{N_{T}}} .
\end{aligned}
$$


Now Equation (A.37) can be expressed as

$$
\begin{aligned}
\mu_{n}^{(s)}(d) & \\
= & \frac{1}{N_{T}} \sum_{k=0}^{N_{T}-1} \sum_{a=1}^{N_{A}} P_{k}^{(s, a)} \\
& {\left[\bar{R}_{k}^{(a)}+\left(\operatorname{SM}[d]-\bar{v}_{n}^{(s)}\right) H_{k}^{(s, a)} \mathrm{e}^{-j 2 \pi \frac{k n}{N_{T}}}-\bar{R}_{k}^{(a)}\right] \mathrm{e}^{j 2 \pi \frac{k n}{N_{T}}}+\bar{v}_{n}^{(s)} } \\
= & \left(\mathrm{SM}[d]-\bar{v}_{n}^{(s)}\right) \frac{1}{N_{T}} \sum_{k=0}^{N_{T}-1} \sum_{a=1}^{N_{A}} P_{k}^{(s, a)} H_{k}^{(s, a)}+\bar{v}_{n}^{(s)} \\
= & \left(\mathrm{SM}[d]-\bar{v}_{n}^{(s)}\right) q_{0}^{(s)}+\bar{v}_{n}^{(s)},
\end{aligned}
$$

where

$$
q_{0}^{(s)}=\frac{1}{N_{T}} \sum_{k=0}^{N_{T}-1} \sum_{a=1}^{N_{A}} P_{k}^{(s, a)} H_{k}^{(s, a)} .
$$

From Equation A.9 and Equation A.40 we know

$$
\begin{aligned}
z_{n}^{(s)}- & \mu_{n}^{(s)}(d) \\
= & \frac{1}{N_{T}} \sum_{k=0}^{N_{T}-1} \sum_{a=1}^{N_{A}} P_{k}^{(s, a)}\left[R_{k}^{(a)}-\bar{R}_{k}^{(a)}\right] \mathrm{e}^{j 2 \pi \frac{k n}{N_{T}}}+\bar{v}_{n}^{(s)} \\
& -\left[\left(\mathrm{SM}[d]-\bar{v}_{n}^{(s)}\right) q_{0}^{(s)}+\bar{v}_{n}^{(s)}\right] \\
= & \frac{1}{N_{T}} \sum_{k=0}^{N_{T}-1} \sum_{a=1}^{N_{A}} P_{k}^{(s, a)}\left[R_{k}^{(a)}-\bar{R}_{k}^{(a)}\right] \mathrm{e}^{j 2 \pi \frac{k n}{N_{T}}} \\
& -\frac{1}{N_{T}} \sum_{k=0}^{N_{T}-1} \sum_{a=1}^{N_{A}} P_{k}^{(s, a)}\left(\mathrm{SM}[d]-\bar{v}_{n}^{(s)}\right) H_{k}^{(s, a)} \\
= & \frac{1}{N_{T}} \sum_{k=0}^{N_{T}-1} \sum_{a=1}^{N_{A}} P_{k}^{(s, a)}\left[R_{k}^{(a)}-\left(\bar{R}_{k}^{(a)}+\left(\mathrm{SM}[d]-\bar{v}_{n}^{(s)}\right) H_{k}^{(s, a)} \mathrm{e}^{-j 2 \pi \frac{k n}{N_{T}}}\right)\right] \\
& \times \mathrm{e}^{j 2 \pi \frac{k n}{N_{T}}} \\
= & \frac{1}{N_{T}} \sum_{k=0}^{N_{T}-1} \sum_{a=1}^{N_{A}} P_{k}^{(s, a)}\left(R_{k}^{(a)}-\mathcal{E}\left[R_{k}^{(a)} \mid d_{n}^{(s)}=d\right]\right) \mathrm{e}^{j 2 \pi \frac{k n}{N_{T}}},
\end{aligned}
$$

where $\mathcal{E}\left[R_{k}^{(a)} \mid d_{n}^{(s)}=d\right]$ is defined at Equation A.39. Therefore,

$$
\sigma_{z, n, s}^{2}=\mathcal{E}\left[\left|z_{n}^{(s)}-\mu_{n}^{(s)}(d)\right|^{2} \mid d_{n}^{(s)}=d\right]
$$




$$
\begin{aligned}
= & \frac{1}{\left(N_{T}\right)^{2}} \sum_{k=0}^{N_{T}-1} \sum_{a=1}^{N_{A}} \sum_{l=0}^{N_{T}-1} \sum_{b=1}^{N_{A}} P_{k}^{(s, a)} P_{l}^{(s, b) *} \\
& \operatorname{Cov}\left[R_{k}^{(a)}, R_{l}^{(b)} \mid d_{n}^{(s)}=d\right] \mathrm{e}^{j 2 \pi \frac{(k-l) n}{N_{T}}}
\end{aligned}
$$

where

$$
\begin{aligned}
\operatorname{Cov}[ & \left.R_{k}^{(a)}, R_{l}^{(b)} \mid d_{n}^{(s)}=d\right] \\
= & \mathcal{E}\left[\left(R_{k}^{(a)}-\mathcal{E}\left[R_{k}^{(a)} \mid d_{n}^{(s)}=d\right]\right)\left(R_{l}^{(b)}-\mathcal{E}\left[R_{l}^{(b)} \mid d_{n}^{(s)}=d\right]\right)^{*} \mid d_{n}^{(s)}=d\right] \\
= & \sum_{u=1}^{N_{S}} \sum_{u^{\prime}=1}^{N_{S}} H_{k}^{(u, a)} H_{l}^{\left(u^{\prime}, b\right) *} \operatorname{Cov}\left[V_{k}^{(u)}, V_{l}^{\left(u^{\prime}\right)} \mid d_{n}^{(s)}=d\right]+\mathcal{E}\left[W_{k}^{(a)} W_{l}^{(b) *}\right] \\
= & \sum_{u=1}^{N_{S}} \sum_{u^{\prime}=1}^{N_{S}} H_{k}^{(u, a)} H_{l}^{\left(u^{\prime}, b\right) *} \sum_{m=0}^{N_{T}-1} \sum_{m^{\prime}=0}^{N_{T}-1} \operatorname{Cov}\left[v_{m}^{(u)}, v_{m^{\prime}}^{\left(u^{\prime}\right)} \mid d_{n}^{(s)}=d\right] \\
& \times \mathrm{e}^{-j 2 \pi \frac{\left(k m-l m^{\prime}\right)}{N_{T}}}+N_{T} N_{0} \delta_{k-l} \delta_{a-b} \\
= & \sum_{u=1}^{N_{S}} \sum_{u^{\prime}=1}^{N_{S}} H_{k}^{(u, a)} H_{l}^{\left(u^{\prime}, b\right) *} \sum_{m=0}^{N_{T}-1} \sum_{m^{\prime}=0}^{N_{T}-1} \operatorname{Cov}\left[v_{m}^{(u)}, v_{m^{\prime}}^{\left(u^{\prime}\right)} \mid d_{n}^{(s)}=d\right] \\
& \delta_{m-m^{\prime}} \delta_{u-u^{\prime}} \mathrm{e}^{-j 2 \pi \frac{\left(k m-l m^{\prime}\right)}{N_{T}}}+N_{T} N_{0} \delta_{k-l} \delta_{a-b} \\
= & \sum_{u=1}^{N_{S}} H_{k}^{(u, a)} H_{l}^{(u, b) *} \sum_{m=0}^{N_{T}-1} \operatorname{Cov}\left[v_{m}^{(u)}, v_{m}^{(u)} \mid d_{n}^{(s)}=d\right] \mathrm{e}^{-j 2 \pi \frac{(k-l) m}{N_{T}}} \\
& +N_{T} N_{0} \delta_{k-l} \delta_{a-b},
\end{aligned}
$$

in which

$$
\begin{aligned}
& \operatorname{Cov}\left[v_{m}^{(u)}, v_{m}^{(u)} \mid d_{n}^{(s)}=d\right] \\
& =\mathcal{E}\left[\left|v_{m}^{(u)}-\mathcal{E}\left[v_{m}^{(u)} \mid d_{n}^{(s)}=d\right]\right|^{2} \mid d_{n}^{(s)}=d\right] \\
& = \begin{cases}\mathcal{E}\left[\left|v_{m}^{(u)}-\bar{v}_{m}^{(u)}\right|^{2}\right] & \text { if } m \neq n \text { or } s \neq u \\
\mathcal{E}\left[\left|v_{n}^{(s)}-\mathrm{SM}[d]\right|^{2} \mid d_{n}^{(s)}=d\right] & \text { if } m=n \text { or } s=u\end{cases} \\
& = \begin{cases}\sigma_{v, m, u}^{2} & \text { if } m \neq n \text { or } s \neq u \\
\mathcal{E}\left[|\operatorname{SM}[d]-\mathrm{SM}[d]|^{2}\right] & \text { if } m=n \text { or } s=u\end{cases} \\
& =\sigma_{v, m, u}^{2}\left(1-\delta_{m-n} \delta_{s-u}\right) .
\end{aligned}
$$


Therefore, Equation A.44 can be simplified as

$$
\begin{aligned}
\operatorname{Cov}[ & \left.R_{k}^{(a)}, R_{l}^{(b)} \mid d_{n}^{(s)}=d\right] \\
= & \sum_{u=1}^{N_{S}} H_{k}^{(u, a)} H_{l}^{(u, b) *} \sum_{m=0}^{N_{T}-1} \sigma_{v, m, u}^{2}\left(1-\delta_{m-n} \delta_{s-u}\right) \mathrm{e}^{-j 2 \pi \frac{(k-l) m}{N_{T}}} \\
& +N_{T} N_{0} \delta_{k-l} \delta_{a-b}
\end{aligned}
$$

and Equation A.43 as

$$
\begin{aligned}
\sigma_{z, n, s}^{2} & \frac{1}{\left(N_{T}\right)^{2}} \sum_{k=0}^{N_{T}-1} \sum_{a=1}^{N_{A}} \sum_{l=0}^{N_{T}-1} \sum_{b=1}^{N_{A}} P_{k}^{(s, a)} P_{l}^{(s, b) *}\left[\sum_{u=1}^{N_{S}} H_{k}^{(u, a)} H_{l}^{(u, b) *}\right. \\
& \left.\sum_{m=0}^{N_{T}-1} \sigma_{v, m, u}^{2} \mathrm{e}^{-j 2 \pi \frac{(k-l) m}{N_{T}}}+N_{T} N_{0} \delta_{k-l} \delta_{a-b}-H_{k}^{(s, a)} H_{l}^{(s, b) *} \sigma_{v, n, s}^{2} \mathrm{e}^{-j 2 \pi \frac{(k-l) n}{N_{T}}}\right] \\
& \mathrm{e}^{j 2 \pi \frac{(k-l) n}{N_{T}}} .
\end{aligned}
$$

For convenience, we may define

$$
\begin{aligned}
& \bar{\sigma}_{z, s}^{2} \\
& =\frac{1}{N_{T}} \sum_{n=0}^{N_{T}-1} \sigma_{z, n, s}^{2} \\
& =\frac{1}{\left(N_{T}\right)^{2}} \sum_{k=0}^{N_{T}-1} \sum_{a=1}^{N_{A}} \sum_{l=0}^{N_{T}-1} \sum_{b=1}^{N_{A}} P_{k}^{(s, a)} P_{l}^{(s, b) *} \sum_{u=1}^{N_{S}} H_{k}^{(u, a)} H_{l}^{(u, b) *} \\
& \sum_{m=0}^{N_{T}-1} \sigma_{v, m, u}^{2} \mathrm{e}^{-j 2 \pi \frac{(k-l) m}{N_{T}}} \frac{1}{N_{T}} \sum_{n=0}^{N_{T}-1} \mathrm{e}^{j 2 \pi \frac{(k-l) n}{N_{T}}} \\
& -\frac{1}{N_{T}} \sum_{k=0}^{N_{T}-1} \sum_{a=1}^{N_{A}} P_{k}^{(s, a)} H_{k}^{(s, a)} \frac{1}{N_{T}} \sum_{l=0}^{N_{T}-1} \sum_{b=1}^{N_{A}} P_{l}^{(s, b) *} H_{l}^{(s, b) *} \frac{1}{N_{T}} \sum_{n=0}^{N_{T}-1} \sigma_{v, n, s}^{2} \\
& +\frac{1}{\left(N_{T}\right)^{2}} \sum_{k=0}^{N_{T}-1} \sum_{a=1}^{N_{A}} \sum_{l=0}^{N_{T}-1} \sum_{b=1}^{N_{A}} P_{k}^{(s, a)} P_{l}^{(s, b) *} N_{T} N_{0} \delta_{k-l} \delta_{a-b} \frac{1}{N_{T}} \sum_{n=0}^{N_{T}-1} \mathrm{e}^{j 2 \pi \frac{(k-l) n}{N_{T}}} \\
& =\frac{1}{\left(N_{T}\right)^{2}} \sum_{k=0}^{N_{T}-1} \sum_{a=1}^{N_{A}} \sum_{l=0}^{N_{T}-1} \sum_{b=1}^{N_{A}} P_{k}^{(s, a)} P_{l}^{(s, b) *} \sum_{u=1}^{N_{S}} H_{k}^{(u, a)} H_{l}^{(u, b) *} \\
& \sum_{m=0}^{N-1} \sigma_{v, m, u}^{2} \mathrm{e}^{-j 2 \pi \frac{(k-l) m}{N}} \delta_{k-l}-\left|q_{0}^{(s)}\right|^{2} \frac{1}{N_{T}} \sum_{n=0}^{N_{T}-1} \sigma_{v, n, s}^{2}
\end{aligned}
$$




$$
\begin{aligned}
& +\frac{1}{\left(N_{T}\right)^{2}} \sum_{k=0}^{N_{T}-1} \sum_{a=1}^{N_{A}} \sum_{l=0}^{N_{T}-1} \sum_{b=1}^{N_{A}} P_{k}^{(s, a)} P_{l}^{(s, b) *} N_{T} N_{0} \delta_{k-l} \delta_{a-b} \delta_{k-l} \\
& =\frac{1}{N_{T}} \sum_{l=0}^{N_{T}-1} \sum_{b=1}^{N_{A}} P_{l}^{(s, b) *} \sum_{a=1}^{N_{A}} P_{l}^{(s, a)} \sum_{u=1}^{N_{S}} H_{l}^{(u, a)} H_{l}^{(u, b) *} \frac{1}{N_{T}} \sum_{m=0}^{N_{T}-1} \sigma_{v, m, u}^{2} \\
& \quad-\left|q_{0}^{(s)}\right|^{2} \frac{1}{N_{T}} \sum_{n=0}^{N_{T}-1} \sigma_{v, n, s}^{2}+\frac{1}{N_{T}} \sum_{l=0}^{N_{T}-1} \sum_{b=1}^{N_{A}} P_{l}^{(s, b) *} \sum_{a=1}^{N_{A}} P_{l}^{(s, a)} N_{0} \delta_{a-b} \\
& =\frac{1}{N_{T}} \sum_{l=0}^{N_{T}-1} \sum_{b=1}^{N_{A}} P_{l}^{(s, b) *} \sum_{a=1}^{N_{A}} P_{l}^{(s, a)}\left[\sum_{u=1}^{N_{S}} H_{l}^{(u, a)} H_{l}^{(u, b) *} \bar{\sigma}_{v, u}^{2}+N_{0} \delta_{a-b}\right] \\
& \quad-\left|q_{0}^{(s)}\right|^{2} \bar{\sigma}_{v, s}^{2} .
\end{aligned}
$$

Noticing the content inside the brackets can be simplified by Equation A.28, we can obtain

$$
\begin{aligned}
\bar{\sigma}_{z, s}^{2} & =\frac{1}{N_{T}} \sum_{l=0}^{N_{T}-1} \sum_{b=1}^{N_{A}} P_{l}^{(s, b) *}\left[H_{l}^{(s, b) *} \bar{\sigma}_{v, s}^{2}\right]-\left|q_{0}^{(s)}\right|^{2} \bar{\sigma}_{v, s}^{2} \\
& =q_{0}^{(s) *} \bar{\sigma}_{v, s}^{2}-\left|q_{0}^{(s)}\right|^{2} \bar{\sigma}_{v, s}^{2} \\
& =\bar{\sigma}_{v, s}^{2} q_{0}^{(s) *}\left(1-q_{0}^{(s)}\right)
\end{aligned}
$$

\section{A.3 Computation Stability}

From Equation (A.32), we know the detector coefficients $P_{l}^{(s, a)}$ are proportional to $\bar{\sigma}_{v, s}^{2}$. In practice, $\bar{\sigma}_{v, s}^{2}$ may be very small, which causes small detector coefficients in absolute values. Such small absolute values may cause very large rounding errors in computer simulations. In order to avoid this ill-conditioned situation, we can 
introduce new detector coefficients $P_{l}^{(s, a)}$ such that

$$
\left[\begin{array}{c}
P_{l}^{(s, 1)} \\
P_{l}^{(s, 2)} \\
\vdots \\
P_{l}^{\left(s, N_{A}\right)}
\end{array}\right]=\left[\begin{array}{cccc}
\Gamma_{l}^{(1,1)} & \Gamma_{l}^{(1,2)} & \ldots & \Gamma_{l}^{\left(1, N_{A}\right)} \\
\Gamma_{l}^{(2,1)} & \Gamma_{l}^{(2,2)} & \ldots & \Gamma_{l}^{\left(2, N_{A}\right)} \\
\vdots & \vdots & \ddots & \vdots \\
\Gamma_{l}^{\left(N_{A}, 1\right)} & \Gamma_{l}^{\left(N_{A}, 2\right)} & \ldots & \Gamma_{l}^{\left(N_{A}, N_{A}\right)}
\end{array}\right]^{-1}\left[\begin{array}{c}
H_{l}^{(s, 1) *} \\
H_{l}^{(s, 2) *} \\
\vdots \\
H_{l}^{\left(s, N_{A}\right) *}
\end{array}\right]
$$

We can also re-define the detector output originally from Equation A.9 as

$$
z_{n}^{(s)}=\frac{1}{N_{T}} \sum_{k=0}^{N_{T}-1} \sum_{a=1}^{N_{A}} P_{k}^{(s, a)}\left(R_{k}^{(a)}-\bar{R}_{k}^{(a)}\right) \mathrm{e}^{j 2 \pi \frac{k n}{N_{T}}}+q_{0}^{(s)} \bar{v}_{n}^{(s)},
$$

where

$$
q_{0}^{(s)}=\frac{1}{N_{T}} \sum_{k=0}^{N_{T}-1} \sum_{a=1}^{N_{A}} P_{k}^{(s, a)} H_{k}^{(s, a)}
$$

Furthermore, the mean of $z_{n}^{(s)}$ under the condition that $d_{n}^{(s)}=d$ can be expressed as

$$
\mu_{n}^{(s)}(d)=q_{0}^{(s)} \mathrm{SM}[d]
$$

and the variance of $z_{n}^{(s)}$ under the condition that $d_{n}^{(s)}=d$ as

$$
\bar{\sigma}_{z, s}^{2}=q_{0}^{(s) *}\left(1-\bar{\sigma}_{v, s}^{2} q_{0}^{(s)}\right)
$$

The branch metrics from this modified model are the same as those from the original model. 


\section{References}

[1] T.S. Rappaport, Wireless Communications: Principles and Practice, (second edition), New Jersey, Prentice-Hall PTR, 2002.

[2] K. Terplan (Ed.), P. Morreale (Ed.), The Telecommunications Handbook, Boca Raton, CRC Press, 2000.

[3] J. Proakis, Digital Communications, (fourth edition), New York, McGraw-Hill, 2001.

[4] R. W. Chang and J. C. Hancock, "On receiver structures for channels having memory", IEEE Transactions on Information Theory, vol. 12, pp. 463-468, Oct. 1966.

[5] C. Douillard, A. Picat, P. Didier, M. Jezequel, C. Berrou and A. Glavieux, "Iterative correction of intersymbol interference: turbo equalization", European Transaction on Telecommunications, vol. 6, pp. 507-511, Sept./Oct. 1995.

[6] A. Glavieux, C. Laot and J. Labat, "Turbo equalization over a frequency selective channel", International Symposium On Turbo Codes, Brest, France, Sept. 1997, pp. 96-102.

[7] M. Tüchler, R. Koetter and A.C. Singer, "Iterative correction of ISI via equalization and decoding with priors", IEEE International Symposium on Information Theory, 2000. Proceedings, June 2000, pp. 100.

[8] M. Tüchler, A.C. Singer and R. Koetter, "Minimum mean square error equalization using a priori information", IEEE Transactions on Signal Processing, vol. 50, pp. 673-683, March 2002.

[9] M.V. Clark, "Adaptive frequency-domain equalization and diversity combining for broadband wireless communications", IEEE Journal on Selected Areas in Communications, vol. 16, issue 8, pp. 1385- 1395, Oct. 1998.

[10] D. Falconer, S.L. Ariyavisitakul, A. Benyamin-Seeyar and B. Eidson, "Frequency domain equalization for single-carrier broadband wireless system", IEEE Communications Magazine, vol. 40, issue 4, pp. 58-66, April 2002.

[11] M. Tüchler and J. Hagenauer, "Turbo equalization using frequency domain equalizers", Proc. Allerton Conf., Monticello, IL, USA, Oct. 2000. 
[12] M. Tüchler and J. Hagenauer, "Linear time and frequency domain turbo equalization", Vehicular Technology Conference, vol. 2, pp. 1449-1453, May 2001.

[13] G.J. Foschini, "Layered space-time architecture for wireless communication in a fading environment when using multi-element antennas", Bell Labs Technical Journal, vol. 1, pp. 41-59, Autumn 1996.

[14] K.K. Wong, R.D. Murch, K.B. Letaief, "Optimizing time and space MIMO antenna system for frequency selective fading channels", IEEE Journal on Selected Areas in Communications, vol. 19, issue 7, pp. 1395-1407, July 2001.

[15] T. Abe and T. Matsumoto, "Space-time turbo equalization in frequency-selective MIMO channels", IEEE Transactions on Vehicular Technology, vol. 52, issue 3, pp. 469-475, May 2003.

[16] J. Lin, Iterative Detection/Equalization with Frequency Domain Filtering for MIMO Channels, Master's thesis, Carleton University, May 2004.

[17] V. Erceg, K.K.S. Hari, M.S. Smith, K.P. Sheikh, C. Tappenden, J.M. Costa, D.S. Baum and C. Bushue, "Channel models for fixed wireless applications", IEEE 802.16. Proposal 802.16.3c-01/29r4.

[18] L.R. Bahl, J. Cocke, F. Jelinek and J. Raviv, "Optimal decoding of linear codes for minimizing symbol error rate", IEEE Transactions on Information Theory, vol. 20, pp. 284-287, March 1974. 